\title{
INVESTIGATING THE FUNCTION OF CLU IN ALZHEIMER'S DISEASE IN IN VITRO AND IN VIVO MODELS
}

\author{
By Tanisha Vithal
}

\author{
A thesis \\ submitted to Victoria University of Wellington \\ in part fulfilment of the requirement for the degree of \\ Master of Science \\ Biotechnology
}

2016

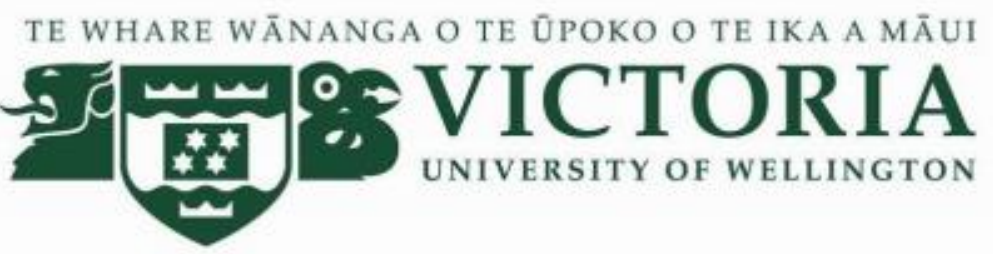


2| P a g e 


\begin{abstract}
Alzheimer's disease (AD) is a neurodegenerative disease that is responsible for $50-80 \%$ of dementia cases and is characterised by lack of visuospatial perception, impairment of language and memory. One of the main physiological attributions towards this disease is the accumulation of large insoluble deposits of amyloid beta, a toxic peptide, which results in the generation of amyloid plaques found in between neurons in the brain. Currently no therapeutic treatments are available. Clusterin (CLU) is an apolipoprotein that when defective is the second highest genetic risk factor for AD. It has been strongly debated whether CLU counteracts or promotes AD pathology. With the roles of CLU including but not limited to acting as a chaperone for cholesterol transport and aiding autophagy functionality in cancer models, this thesis investigates these two specific functionalities by overexpressing CLU in an in vitro SH-SY5Y and in an in vivo $A D$ model of Drosophila melanogaster (fruit fly). Conclusions from this study reveal that within D. melanogaster, CLU reduced $A \beta 42$ levels and increased cholesterol effect through the blood brain barrier. Additionally, in human cells, CLU ameliorated the defective flux in autophagy. This thesis sheds light into how CLU plays a protective role within an Alzheimer's disease mammalian system.
\end{abstract}




\section{ACKNOWLEDGEMENTS}

I would like to give my thanks to Dr Andrew Munkacsi for being a great supervisor and being a pleasure to work with throughout this project. Thank you for your support, time and faith you have put into me and this thesis.

I would also like to thank Helen Fitsimmons (Massey University, Palmerston North) for research collaboration with this project and the aid of developing the overexpressed CLU Alzheimer's disease fly model. You have been a tremendous help with this part of my project and I'll be forever grateful.

Thank you to Dr David Ackerley for aiding me in the design of the mammalian GFP primers used for in vitro work to detect CLU within SH-SY5Y cell lines.

I would also like to thank everyone in the Chemical Genetics Laboratory at Victoria University for your friendship, kindness, advice and support through the good and bad times. I especially like to note the contributions made by Remy Schneider for help with confocal imaging and advice, as well as Dinidu Senanayake for help in data analysis.

Finally I would like to give a huge thank you to my family, my mum, dad, and brothers for always being there for me. For feeding me, for putting up with my stress levels, and unconditionally loving me regardless of the mood swings that they might have encountered. You have always supported me regardless of the situation, and I couldn't be luckier to have you all in my life. 
TABLE OF CONTENTS

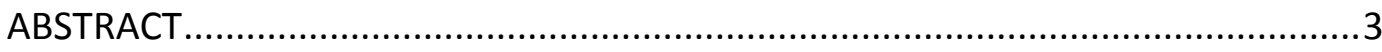

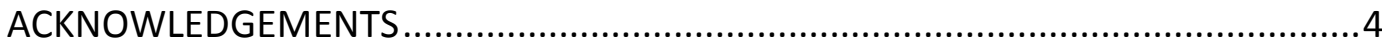

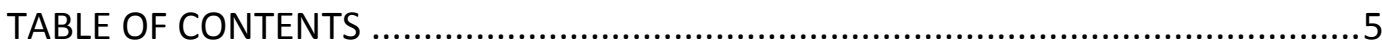

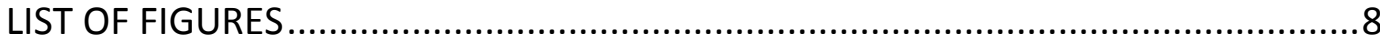

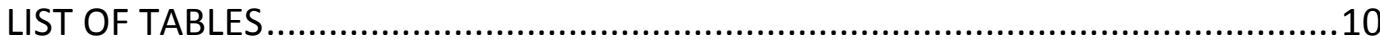

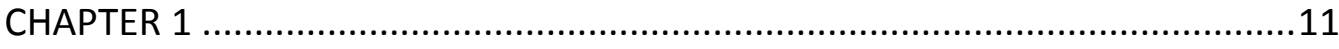

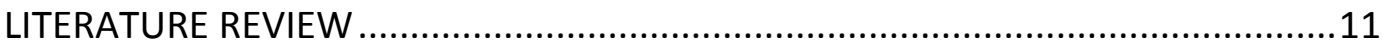

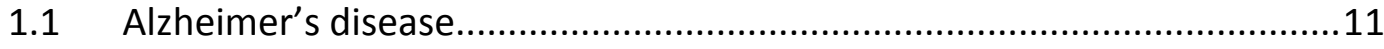

1.2 Cholesterol and lipid metabolism in Alzheimer's disease ........................14

1.3 Autophagy and Alzheimer's Disease ...................................................... 17

1.4 Cholesterol and Autophagy in Alzheimer's disease.................................20

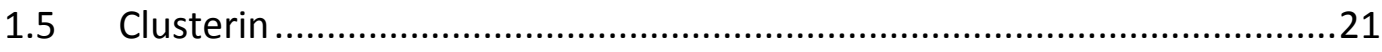

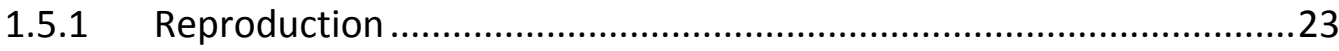

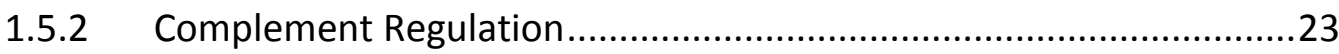

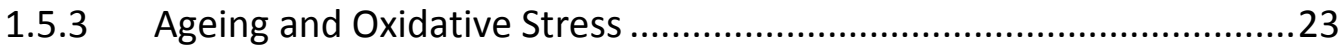

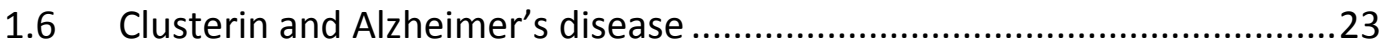

1.7 Clusterin-mediated Lipid Metabolism ....................................................25

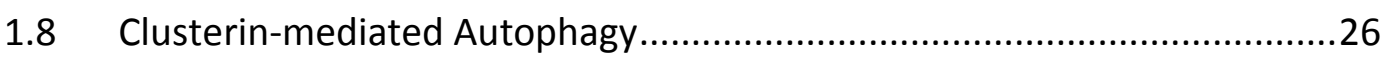

1.9 Model Organisms to Investigate Alzheimer's Disease..............................26

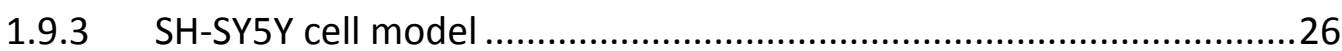

1.9.4 Fruit fly Model of Alzheimer's disease .............................................27

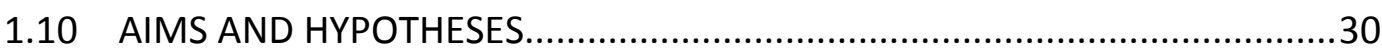

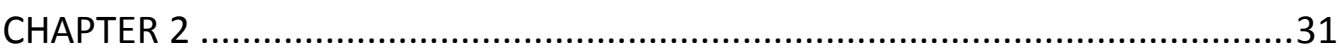

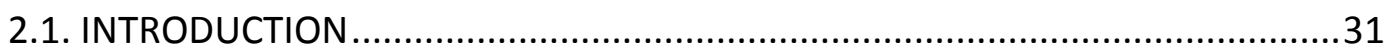

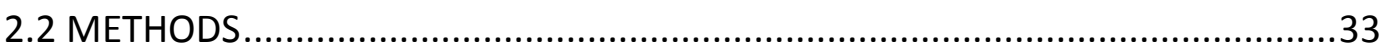

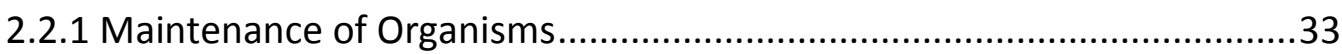

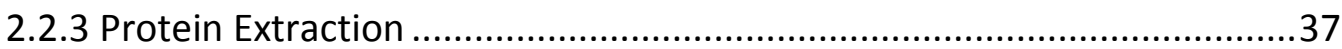

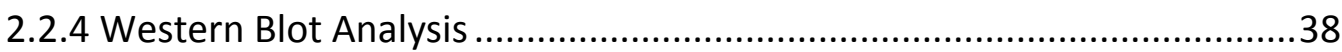

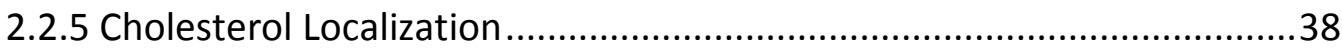

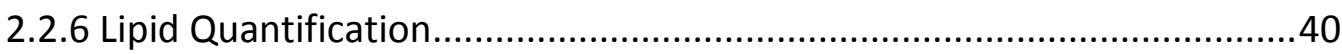

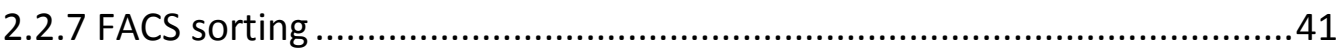

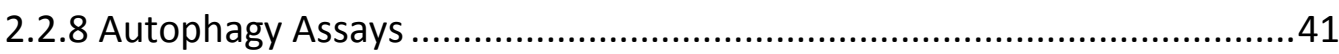


2.3.1 Generation of UASCLU Drosophila/hCLU DNA

2.3.1.1 Cloning

2.3.1.2 Creation of pDNR-Dual + corrected version clusterin construct .......42

2.3.1.3 Creation of pUAST-attB-CLU construct .47

2.3.1.4 Generation of the UAS-CLU Drosophila melanogaster strain 47

2.3.1.5 Generation of UAS-APP695-BACE-CLU Drosophila melanogaster strains .49

2.3.2. Generation of CLU in SH-SY5Y neuroblastoma cells 52

2.3.1.1 Cloning .52

2.3.1.2 Creation of pcDNA3.1(+) + clusterin construct .52

2.3.1.3 Integration of GFP in pcDNA3.1(+)/CLU construct .54

2.3.1.4 Transfection optimisation of pcDNA3.1(+)/CLU/GFP in SH-SY5Y cell lines

2.4 Clusterin Enhances Survival Rates in the APP/BACE Fruit fly Model of Alzheimer's disease.

2.5 Clusterin Ameliorates the Crumpled Wing Phenotype in the APP/BACE Fruit fly Model of Alzheimer's disease.

2.6 Clusterin Reduces Full Length APP and $A \beta_{42}$ levels in SWAPP SH-SY5Y cells but not in the APP/BACE fly

2.7 Clusterin Mobilizes Cholesterol Aggregates in the APP/BACE Fly Model of Alzheimer's Disease

2.8 Clusterin May Reduce Cholesterol levels in the Brains of the APP/BACE Fly Model of Alzheimer's Disease ..................................................................... 78

2.9 Clusterin Disrupts Autophagic Flux in SH-SY5Y+ swAPP Cells .81

2.10 Lysotracker red staining reveals overexpression of clusterin results in visual change but no statistical change lysosomal acidification levels within sWAPP SH-SY5Y Alzheimer's disease cell model....

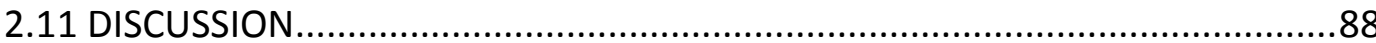

2.11.1 Comparison of in vitro and in vivo models of Alzheimer's disease ........88

2.11.2 Effects of clusterin on Alzheimer's disease pathology ..........................89

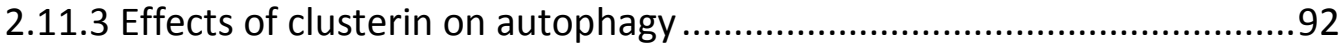

2.11.4 Effects of clusterin on cholesterol metabolism ..................................93

2.11.5 Proposed mechanism of action of CLU in Alzheimer's disease ..............96

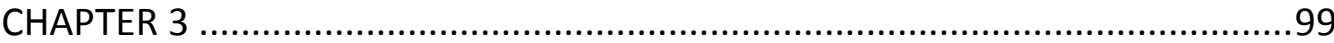

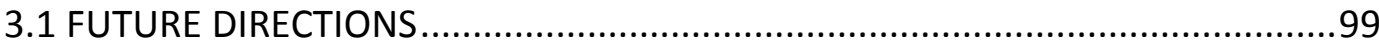

3.1.1 Further Characterisation of the Effect of CLU on Alzheimer's disease Pathology 
3.1.2 Further Investigation into the Effect of CLU on Cholesterol Metabolism

3.1.4 Further Characterization of the Effect of CLU on Autophagy.................104

3.1.5 Additional Mechanisms to Pursue ........................................................105

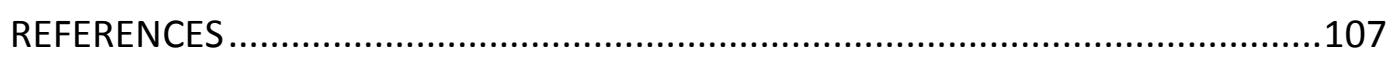

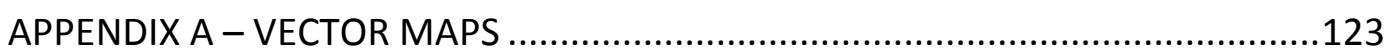

Figure A1 - Corrected pDNR-Dual hCLU plasmid ........................................123

Figure A2 - pUAST-attB plasmid..............................................................124

Figure A3 - pUAST-attb hCLU plasmid......................................................125

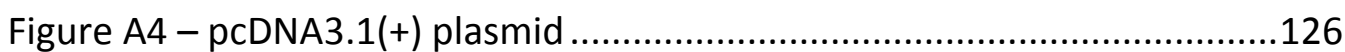

Figure A5 - RG203629 GFP plasmid .......................................................127 


\section{LIST OF FIGURES}

Figure 1.1: Processing of APP by non-amyloidogenic and amyloidogenic pathways 12

Figure 1.2: Cholesterol and APOE in regulation of amyloid beta fragments.........14

Figure 1.3: Lipid mechanisms that influence APP processing. ...........................15

Figure 1.4: Induction of autophagy and autophagosome generation ..................18

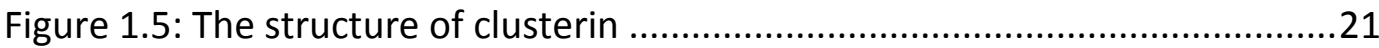

Figure 1.6: The GAL4/UAS (upstream activating sequence) system in $D$.

melanogaster .28

Figure 2.1: Sequence confirmation of corrected CLU fragment within pDNR-Dual

$+\mathrm{CLU}$ .44

Figure 2.2: Correction of CLU mutation in pDNR-Dual + CLU vector. .46

Figure 2.3: Generation of APP695/BACE/CLU D. melanogaster mutants (crosses)

Figure 2.4: Creation of pUAST-attB + CLU vector ..................................................51

Figure 2.5: Creation of pcDNA3.1(+) + CLU construct ........................................53

Figure 2.6: Creation of GFP + pcDNA3.1(+) CLU construct .................................55

Figure 2.7: Experimental method for WT-SHSY5Y transfection optimisation.......57

Figure 2.8: Experimental method for SWAPP SH-SY5Y transfection optimisation 58 Figure 2.9: Transfection trial of WT-SHSY5Y cells incubated for 24 h (well 1-6)...59 Figure 2.10: Transfection trials for WT SHSY5Y cells incubated for $24 \mathrm{~h}$ (well 7-12)

Figure 2.11: Transfection trials of WT SHSY5Y cells incubated for 48 h (well 1-6)61

Figure 2.12: Transfection trials of WT SHSY5Y cells incubated for 48h (well 7-12)

Figure 2.13: Transfection trials of swAPP SH-SY5Y cells incubated for $24 \mathrm{~h}$ (well 16)

Figure 2.14: Transfection trials of swAPP SH-SY5Y cells incubated for $24 \mathrm{~h}$ (well 712) 
Figure 2.15: Transfection optimisation (1:2 DNA:Lipofectamine) in WT SH-SY5Y cells

Figure 2.16: Transfection optimisation (1:2 DNA: Lipofectamine) in SWAPP SHSY5Y cells .66

Figure 2.17: Clusterin rescues morphological crumpled wing defect in APP/BACE AD flies 69

Figure 2.18: CLU prevents cleavage of FL-APP and $A \beta_{42}$ levels in AD cells and no effect in AD D. melanogaster lines

Figure 2.19: Treatment with gamma secretase on swAPP SH-SY5Y cells improves detection on $\beta C T F$ fragment .73

Figure 2.20: Filipin detection reveals decreased free cholesterol content within APP/BACE/CLU fly brains .76

Figure 2.21: Filipin detection reveals increased cholesterol content within sWAPP SH-SY5Y cells .77

Figure 2.22: MALDI-TOF analysis reveals a decrease in overall cholesterol content when $\mathrm{CLU}$ is upregulated in AD flies .79

Figure 2.23: Gating strategy for FACS sorting of GFP/(sWAPP/CLU $\left.{ }^{+/+}\right)$positive cells .80

Figure 2.24: Purity check for FACS sorting of GFP/(swAPP/CLU $\left.{ }^{+/+}\right)$cells 81

Figure 2.25: Clusterin aids lysosomal digestion and decreases autophagosome accumulation in AD SH-SY5Y neuroblastoma cells .83

Figure 2.26: Acidification levels are upregulated within SWAPP SH-SY5Y cells in comparison to WT SH-SY5Y cells .85

Figure 2.27: Overexpression of clusterin reduces lysosomal acidification levels in a SWAPP SH-SY5Y cell line .86

Figure 2.28: Quantification of Lysotracker red SH-SY5Y samples .87

Figure 3.1: Schematic diagram for processes occurring in an AD model 97

Figure 4.1: Immunohistochemistry FASII staining in AD flies reveals hindrance in structural integrit of mushroom bodies .103 


\section{LIST OF TABLES}

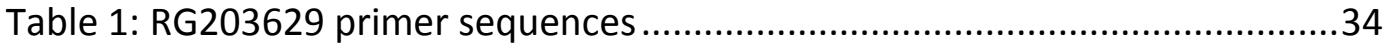

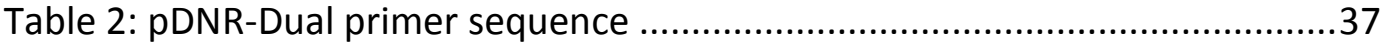




\section{CHAPTER 1}

\section{LITERATURE REVIEW}

\subsection{Alzheimer's disease}

In 2015, there are currently 47.5 million people who live with dementia and within the year, 7.7 million new cases will occur. In 2030, it is estimated that the total number of people with dementia will rise to 75.6 million people.

Alzheimer's disease (AD) is a terminal neurodegenerative disorders that is estimated to contribute $60-70 \%$ of dementia cases ${ }^{153}$.

Unfortunately, no long term therapeutic treatments are currently available ${ }^{153}$. Even though $A D$ manifests as a form of dementia in the elderly with diagnosis of the disease typically occurring around the age of $65^{2}$, this disease is not an underlying cause that naturally affects ageing processes. The progression rate of the $A D$ is variable, but usually occurs over a decade ${ }^{3}$.

Characterisation of the disease includes lack of visuospatial perception and impairment of language function and memory. In the late stages of $A D$, motor abnormalities become increasingly common as simple activities carried out on a daily basis are severely impaired ${ }^{3}$. Physiological attributes associated with $A D$ include the development of intracellular neurofibrillary tangles in the hippocampus, particular subcortical nuclei, cortex and amygdala ${ }^{3}$, loss of function of neurons and synapses, and the build-up of senile plaques ${ }^{4}$ Prior head trauma is also a factor that can contribute to the pathogenesis of $A D^{4}$. With the key contributor of the disease being the accumulation of amyloid plaques in the brain, the dynamics of how this mechanism in $A D$ leads up to such a severe degenerative state within the brain is poorly understood ${ }^{5}$. There are two different modalities of acquiring this particular form of dementia: familial and sporadic $^{5}$. In familial AD, amyloid precursor proteins (APP) undergo two separate types of cleavage (the first cleavage is though $\beta$-secretase and the second by $\gamma^{-}$ secretase) resulting in amyloid beta $(A \beta)$ fragments which is a component of the amyloidogenic pathway (Figure 1), with sporadic consisting the remainder of $A D$ 
Figure 1.1: Processing of APP by non-amyloidogenic and amyloidogenic pathways (Figure taken from Del Prete et al, 2014). Amyloid precursor protein (APP) can be processed by alpha, beta and gamma secretases into fragments which are readily cleared from the body or fragments which promote neurotoxicity and lead to detrimental downstream effects that accelerate the ageing process in the body. In the non-amyloidogenic pathway, APP is processed by alpha and gamma secretase to produce amyloid precursor protein intracellular domain (AICD), p3 and SAPP-alpha which are all degraded and cleared from the body. However in the amyloidogenic pathway, gamma secretase cleaves APP, but alpha-secretase cleavage is replaced by beta-secretase. This results in toxic fragments known as amyloid beta. These plaques build up in the body and contribute to a neurodegenerative state.

cases. It should be noted that alongside plaque accumulation within the body, neurofibrally tangles (NFTs) made up of hyperphosphorylated tau protein are also a key contributor to the disease. Tau destabilises microtubules, which also contribute to neuronal death. It is believed that amyloid beta accumulation leads to NFTs, however other studies question this hypothesis, and instead postulate that the leading cause of $A D$ is actually the inverse, with the presence of tau pathology occurring before amyloid plaque deposits.

The negative implications of $A \beta$ oligomer generation includes the hindrance of long-term potentiation of the hippocampus, obstruction of synaptic function and oxidative stress, and inflammatory stress generated by deposited and aggregated $A \beta$. This in turn leads to neurotransmitter impairments and loss of cognitive function as the disease develops ${ }^{65}$.

Approximately $1 \%$ of $A D$ cases is considered familial and portray autosomal dominant inheritance ${ }^{6}$. 
The amyloid cascade hypothesis states that the balance between these two processes of amyloid build up and clearance is a crucial cycle that needs to be maintained in order to avoid plaque build-up ${ }^{7}$. The familial form of AD has been linked to mutations found in the following genes: APP on chromosome 21 , presenilin 1 (PSEN1) on chromosome 14 and presenilin 2 (PSEN2) on chromosome $1^{3,7}$. These three genes are identified as the causative agents contributing approximately $30-50 \%$ of early onset familial $A D^{3}$.

However, the majority of the disease links to the late-onset sporadic AD (LOAD) that is a consequence of multiple underlying factors ${ }^{7}$. The number one risk factor is the $\epsilon 4$ allele of the apolipoprotein $E(A P O E)$ gene on chromosome $14^{3,7}$. Further studies have confirmed this finding as APOE being a main determinant in the contribution to lowering the age of onset (68 instead of 84) of the disease as well as increasing the risk of $A D(20 \% \text { to } 90 \%)^{7}$. If an individual possesses one copy of the $\epsilon 4$ allele, the extra copy could potentially increase the development of $A D$ from two to five fold. If two copies of the allele are obtained, the probability of increasing the disease further increases to above a five-fold probability $^{3}$.

Previous studies by Lambert et al. (2013), used genome wide association study (GWAS) analyses to determine other genetic variants and factors contributing to the disease. The three major variants that were identified in the clusterin (CLU) gene (on chromosome 8), the complement component (3b/4b) receptor 1 (CR1) gene (on chromosome 1) and the phosphatidylinositol binding clathrin assembly (PICALM) gene (on chromosome 11 ) 3 . Other genes which have been found to play a role in Alzheimer's disease, but to a lesser extent, include bridging integrator 1 (BIN1) gene and sortilin-related receptor 1 (SORL1) gene $^{3}$. Within GWAS studies, CLU, CRI and PICALM are identified in every hit whilst BIN1 and SORL1 are not necessarily produced in every study. As the APOE $\varepsilon 4$ is another gene heavily implicated as the top genes being involved in AD, APOE $\varepsilon 4$ general role in cholesterol homeostasis had lead scientists to examine the role of cholesterol in $A D^{3}$. 


\subsection{Cholesterol and lipid metabolism in Alzheimer's disease}

Even though the brain consists $2 \%$ of an individual's total body mass, the brain holds approximately $25 \%$ of the total body cholesterol ${ }^{3}$. Cholesterol in the brain exists mainly in the membranes of neurons and glial cells, as well as in myelin sheaths in an unesterified form ${ }^{4}$. Cholesterol metabolism is pivotal to synaptic plasticity and neuronal development. Other critical mechanisms that cholesterol regulates include regulation of neurotransmitter release, neurite outgrowth, formation of synapse, and synaptic vesicle transport ${ }^{3}$. In previous studies of the brain examining cholesterol regulation, it has been concluded that

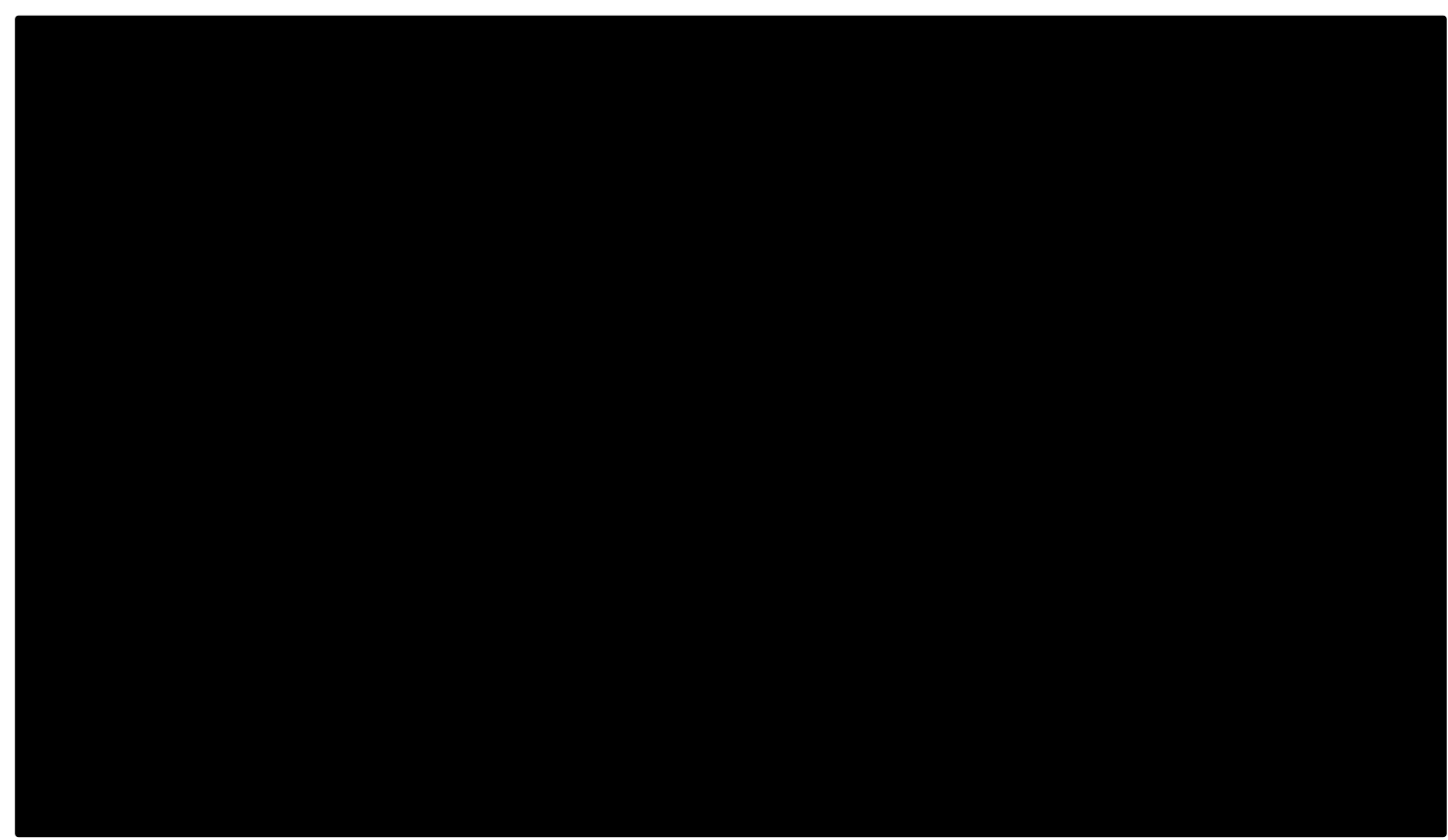

Figure 1.2: Cholesterol and APOE in regulation of amyloid beta fragments

Figure taken from (Paolo and Kim, 2011). Cholesterol in the brain is supplied through either de novo synthesis from the endoplasmic reticulum, (ER) or from outside of the brain in the form of high-density lipoproteins (HDLs) which due to their size can pass through the blood brain barrier. Larger lipoproteins such as very low-density lipoprotein and low density lipoprotein (VLDL/LDL) can't pass. However it should be noted that when LDL receptors are upregulated, plaque clearance is dramatically increased (Kim et al, 2009). HMG-CoA mediates the cholesterol biosynthesis from the ER, whereas ACAT converts free cholesterol to cholesterol esters for storage. When HMG-CoA and ACAT activity are blocked, amyloid beta peptide levels are lowered. APOE linked to HDLs bind to LRP receptor and aids clearance of amyloid beta peptides, whereas free APOE has the opposite effect, in that it aids plaque aggregation. Note that $A \beta$ peptides are generated in the lumen of neuronal organelles, whereas peptides are drawn in the diagram in the cytoplasm for simplicity. 
a balance between cholesterol esters and free cholesterol is vital in controlling amyloidogenesis $^{4}$.

Cholesterol plays an important part contributing to AD. Abnormally high levels of cholesterol have been found in the cores of mature $A \beta$ plaques in postmortem brains of AD patients. These findings have also been reproduced in an AD mouse models expressing the Swedish APP mutation $\left(\right.$ TgAPP $_{S w}$ line 2576) ${ }^{9}$, with both high cholesterol and $A \beta$ absent from normal controls. These results

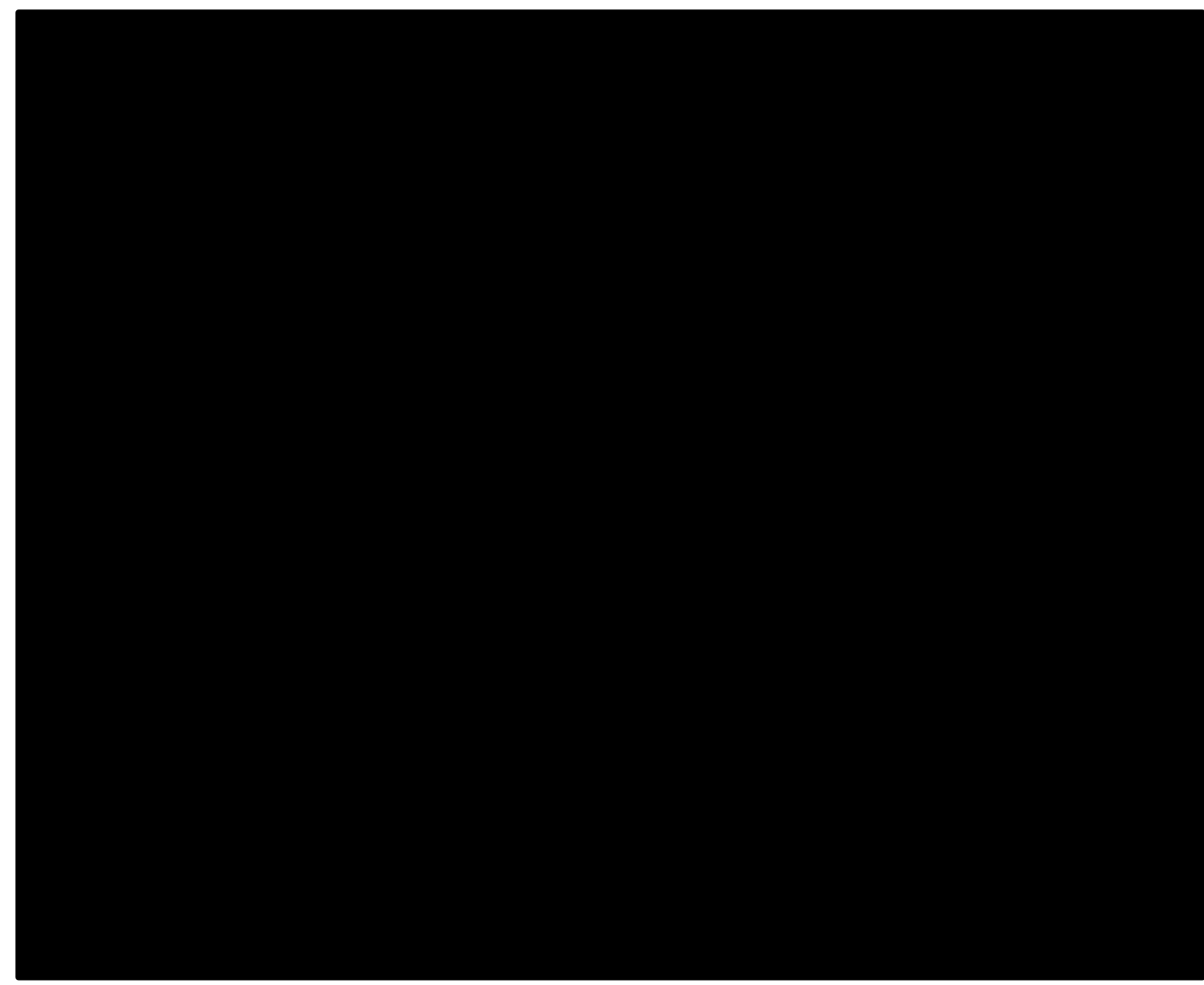

Figure 1.3: Lipid mechanisms that influence APP processing.

Figure taken from (Paolo and Kim, 2011). a. APP is embedded in lipid rafts and processing primarily occurs at this site by beta and gamma secretases to produce amyloid beta peptides. Cholesterol and LRP encourages BACE1 localisation and cleavage in lipid rafts. GGPP (geranylgenranyl pyrophosphate) influences localisation of gamma secretase with lipid rafts. b. Alpha secretase also acts on APP within lipid rafts through the non-amyloidogenic pathway. Alpha-secretase preferentially cleaves in a non-lipid raft environment. Other factors that promote and contribute to the nonamyloidogenic pathways include phospholipase C (PLC), diaglycerol and isoprenoids. c. Particular lipids (shown in the blue boxes) act on BACE and gamma secretase activity. When ceramide and cholesterol increase, BACE1 activation as well as cholesterol and sphingolipid levels increases activity of gamma secretase. However, phospholipase D1 PLD1 and sphingomyelinase SMase negatively regulate gammasecretase levels. 
suggest unusual cholesterol metabolism in the brain which may contribute to $A D$ pathology.

The association between cholesterol, APP processing and amyloid beta regulation in $A D$ has been investigated primarily through regulating diet in in vitro and in vivo models. The first experiment that displayed this association illustrated that within rabbit hippocampal neurons, when fed for high-cholesterol during a four week period, amyloid beta generation is enhanced ${ }^{10}$. Whilst one approach concentrates on upregulating cholesterol for experimental investigations into APP-mediated lipid metabolism, pharmacological drugs have also been used to study effects on APP and amyloid beta in a lowered lipid/cholesterol environment. Cholesterol drugs include, but are not limited to, simvastatin ${ }^{11,12}, \mathrm{BM} 15.766^{13}$, lipitor ${ }^{14}$ and lovastatin ${ }^{15}$ which also have effects in other cholesterol diseases such as atherosclerosis and diabetes.

Complementary to the studies above that regulated cholesterol levels through either diet or drugs to study the effects of cholesterol in AD, additional experiments have focused on manipulating gene expression within genetic models that control cholesterol homeostatic processes.

As mentioned above, APOE, a regulator of cholesterol metabolism in the brain, is the top genetic risk factor for AD. APOE also systemically regulates triglyceride metabolism in the body ${ }^{4}$. In the brain, APOE aids in the uptake of lipoprotein particles through three receptor channels: the very low-density family lipoprotein receptor, the lipoprotein receptor related protein (LRP) and the low-density lipoprotein (LDL) receptor ${ }^{4}$. In AD, APOE is severely hindered and is a contributing factor to this form of dementia ${ }^{3,16}$ such as deregulating cholesterol efflux and autophagic clearance ${ }^{1,3,4}$. As potent as APOE can be in the role of $A D, A P O E$ can be regulated via expression levels of $A B C A 1$, a gene that is critical in the efflux of excess intracellular cholesterol to extracellular lipid chaperones, allowing these chaperones to be lipidated ${ }^{17-19}$ (Figure 1.2).

It has been shown that when APOE is inhibited in APP transgenic mice, this results in reduced APOE levels and in turn increases amyloid beta deposition. Since cholesterol levels increase due to impaired transport by apolipoproteins (e.g., APOE) and subsequently neurons and synapses degenerate, cholesterol 
efflux to and from the blood-brain barrier (BBB) by APOE and other proteins also contributes to amyloid- $\beta$ generation. It should be noted that since mammalian cells can't degrade cholesterol, it must be transported elsewhere in the body to be utilised.

Acyl-coenzyme A: cholesterol acyltransferase (ACAT) is another gene that is implicated in Alzheimer's disease. When ACAT is inhibited using drugs such as CP-113, $818^{8,20,21}$, cholesterol esters decrease as expected and amyloid beta levels also decrease. Deletion of ACAT also increases oxysterol levels of 24(S)-hydroxycholesterol by converting excess free cholesterol in the brain. Oxysterols can then be transported through the BBB, which suggests the balance between cholesterol esters and free cholesterol is a crucial component to lowering abnormal plaque levels in the brain. Cholesterol can also directly influence the behaviour of secretase activities that affect $A \beta$ regeneration. If levels of cholesterol are decreased in the membrane, for example by $\beta M C D(\beta-$ methyl cyclodextrin), activity of BACE1 and $\gamma$-secretase are decreased, which in turn leads to decreased levels of $A \beta$ generation ${ }^{22,23}$. Cholesterol levels also control the $\gamma$-secretase-mediated production of $A \beta^{11}$. These findings elucidate that environments containing high cholesterol directly mediate BACE1 activity. In addition, cholesterol and other lipids such as sphingolipids and diacylglycerol (Figure 1.3) also influence APP processing. Since several studies show correlations between high cholesterol and the increase of $A \beta$ build up along with $A D$ pathology ${ }^{24-26}$, therapeutic approaches need to address lowering excess cholesterol and lipid levels in the brain.

\subsection{Autophagy and Alzheimer's Disease}

Macroautophagy (now referred to as autophagy) is a mechanism within the body that is responsible for degrading, recycling and clearance of damaged organelles and misfolded proteins. It exhibits a protective role and is triggered by either the inhibition of the molecular target of rapamycin (mTOR) pathway or by activation of AMP-activated protein kinase (AMPK). This lysosomal degradative process (Figure 1.4) is one of several steps within the autophagy pathway that ensures 
Figure 1.4: Induction of autophagy and autophagosome generation

Figure adapted fromFerri et al, $2005^{1}$ and Ranbinowitz and White et al, 2010. Macroautophagy (referred to in text as autophagy), is a mechanism which recycles cytoplasm and defective proteins and organelles. First in this process is the generation of an isolation membrane (phagophore), followed by elongation of the phagophore around a selected region of protein and/or cytoplasm which eventually closes to form a double membraned autophagosome. Note that LC3 proteins (LC3I after lipidation converts to LC3II) are incorporated into the inner and outer membrane during this process. The autophagosome will either directly fuse with the lysosome, or first combine with an endosome. The end product will be formation of the autolysosome. Hydrolic enzymes contained mainly within lysosomes will establish efficient enzymatic digestion. Degraded proteins are then released into the cytoplasm where proteins are in a metabolic form that cells can use for energy or used for de novo synthesis of proteins. LC3II proteins are also degraded in this process.

efficient regulation by two processes. The first process is acted by lysosomalassociated membrane protein 1 and 2 (LAMP1 and LAMP2) which prevent lysosomal self-digestion. The second process focuses on the expression and regulation of TFEB (transcription factor EB) and $\mathrm{MTORC1}$ controlled by 'The Ragulator' (a protein complex made up of small GTPases). This acts as an on/off switch for activation or inhibition of autophagy. The dynin-dystan complex also facilitates the efficiency of autophagosome fusions and digestion of intracellular contents by transporting autophagosomes towards the MTOC (microtubule 
organising centre) within neurons in which lysosomes are most abundantly found ${ }^{1}$.

In $A D$, the lysosomal digestion mechanism during autophagic processing is dysfunctional, as autolysosomes and associated autophagic vacuoles accumulate in neuritic swellings in an AD brain ${ }^{27}$. This finding suggests that autophagosomes retain the ability to fuse with lysosomes, however the release of digested substrates from the autolysosome is somewhat impaired. The disruption of autolysosomal proteolysis in wild type mice phenocopies the pathology of that seen in mouse models of Alzheimer's disease, and further disruption of lysosomal proteolysis in these mice aggravates pre-existing pathologies ${ }^{27}$.

Contributions to defective lysosomal digestion include several genetic risk factors associated with AD such as presenilin 1 (PSEN1) and APOE as well as defective proteins such as APP. PSEN1 controls lysosomal acidification through the v-ATPase multimeric enzyme complex. This complex is integrated into the lysosomal membrane and is responsible for downstream effects that regulate protease activity in order to upregulate or downregulate the rate of autophagic processes. With this mechanism that ensures cellular homeostasis in eukaryotic organisms being compromised, disease onset is dramatically accelerated as well as exacerbating the pathological symptoms of $A D^{1}$.

Lysosomal membrane permeabilization (LMP) can also be triggered by amyloid beta build up and the APOE4 variant. Depending on the level of LMP activation, this process can lead to catabolic hydrolases being prematurely released which in turn results in necrosis or apoptosis and neuronal cell death ${ }^{1}$. The APOE4 variant, abnormal cholesterol levels, or APP mutations also influence substrate clearance, as overload of substrates in the lysosome destabilises the autolysosomal membrane. ApoE also affects Rab5 and Rab7 activation. Rab5 is critical for recruiting Rab7 to the phagosome, which is in turn essential for maturation of the endosome and endo-lysosomal trafficking and transport ${ }^{1}$. 


\subsection{Cholesterol and Autophagy in Alzheimer's disease}

Few studies focus on drug interactions between cholesterol and autophagy regulation acting in synchrony to achieve homeostasis within $A D$ models. However drugs such ursolic acid ${ }^{28}$ and $\operatorname{simvastatin}^{29}$ have shed some insight that the two mechanisms are linked. In C57BL/6J mice fed a high-fat diet, administration of ursolic acid activates PPAR-alpha; a factor that controls beta oxidation and fatty acid transport. Activation of PPAR-alpha in turn increased adiponectin, hepatic autophagy, HDL cholesterol and other genes regulating lipogenesis (e.g., SREBP-1c) ${ }^{28}$. However, simvastatin operates through a different mechanism. Within a J774A.1 macrophage cell line, it has been demonstrated that simvastatin can induce therapeutic affects in diseases such as atherosclerosis by inducing autophagy through increasing both oxidised lowdensity lipoprotein, conversion of LC3-I to LC3-II and attenuating cholesterol accumulation $^{29}$.

Given that APOE is a genetic risk factor for Alzheimer's disease, attention has also been concentrated on apolipoproteins. ApoA-I interacts with ABCA1 following lipidation by migration to the plasma membrane to bind cholesterol. Interestingly, lipidation of $A p o A$ is enhanced through suppression of Akt via mTOR inhibition. Furthermore, only functional ABCA1 are affected, with mutant forms of the ABCA1 gene unaffected ${ }^{4}$. In APOE-deficient mice, overexpression of lireglutide (glycogen-like peptide) activates autophagy and exhibits a protective role, including the decrease of LDL-cholesterol and total cholesterol levels ${ }^{30}$. In linking these mechanisms to Alzheimer's disease, the emergence of the impact that cholesterol and autophagy has in this neurodegenerative disease has been rigorously investigated.

Studies examining cholesterol genes as the source of abnormal autophagic regulation have focused on ACAT1, an endoplasmic reticulum enzyme that blocks the cholesterol accumulation within membranes by converting cholesterol to cholesterol esters. A recent study shows that the pharmacological ACAT1 inhibitor K604 can improve $A \beta_{1-42}$ clearance by the autophagic pathway as well as improve packaging of oligometic A $\beta 1-42$ via 
stimulated autophagosome formation. TFEB, one of the regulators that control the expression levels of autophagy is also upregulated by ACAT1 inhibition. Results linking ACAT1 and autophagy have also been demonstrated in an ACAT1 knockout mouse ${ }^{31}$ as well as in a triple mutant mouse model expressing human APP, tau and presenilin-1. Within this study, through inactivation of ACAT1, tau levels also decrease ${ }^{31}$.

Other studies have investigated whether components of the autophagic pathway are the primary cause for defective lipogenesis. For example, deletion of cystatin B (a lysosomal protease inhibitor) in the TgCRND8 mouse model of AD improved clearance of lipid-containing lysosomes via autolysosomes for clearance of neuronal debris, misfolded proteins and excess lipid accumulation ${ }^{32}$.

\subsection{Clusterin}

Clusterin (CLU), also known as APOJ ${ }^{33}, \mathrm{SP} 40-40^{34}, \mathrm{CLI}^{35} \mathrm{TRPM}^{36}{ }^{36}, \mathrm{XIP}^{37}$ and SGP- $2^{38}$, is a stress-induced $80 \mathrm{kDa}$ glycoprotein ${ }^{39}$. CLU is located on chromosome 8 p21 and comprised of nine exons ${ }^{40}$. Clusterin is synthesized by

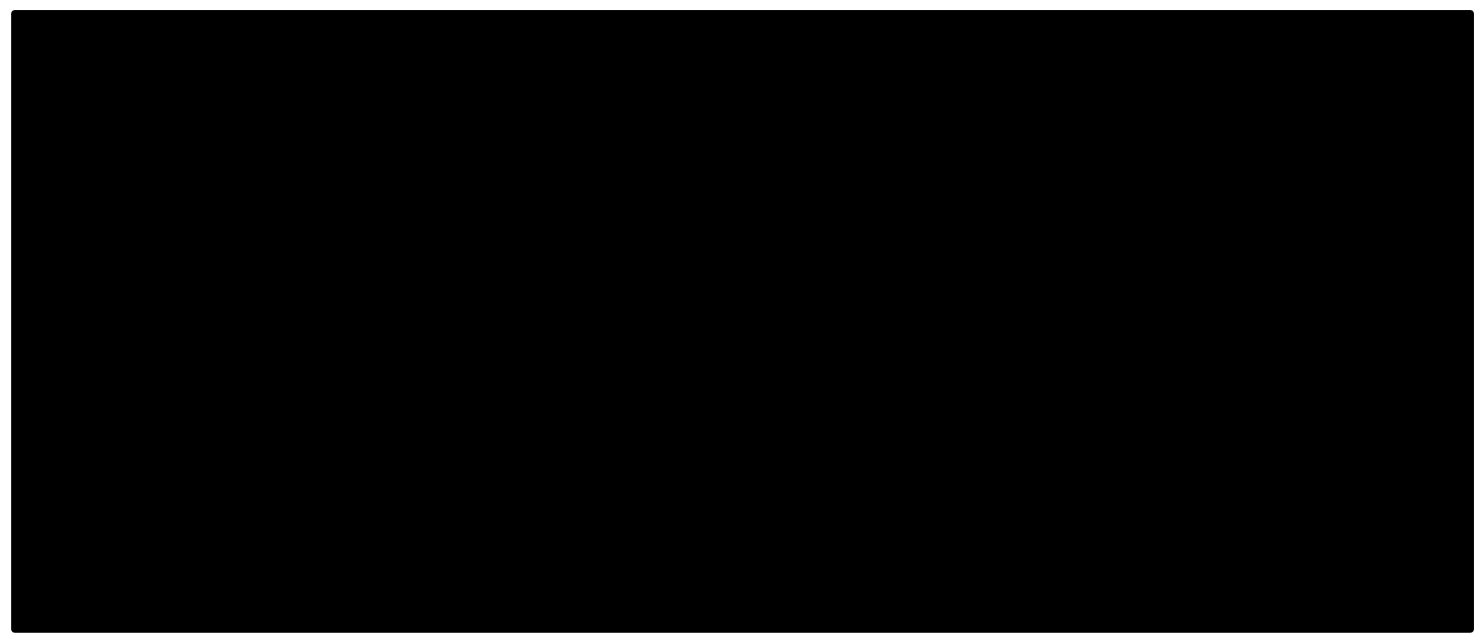

Figure 1.5: The structure of clusterin

The figure was adapted from the following: (Kirszbaum et al., 1989; Jenne and Tschop., 1989; Jordan-Starck et al.; 1992; Jenne and Tschopp., 1992; Fritz and Murphy, 1993; Wong et al., 1994; Rosenberg and Silkensen., 1995; Calero et a.l, 2000). Clusterin is an $80 \mathrm{kDa}$ protein, made up of a heterocomplex consisiting of an alpha chain, containing 205 amino acids, and beta chain consisting of 222 amino acids. Based from literature reports, each chain consists of four amphipathic helices (indicated by orange regions), four heparin binding domains (indicated by black circles), and three $\mathrm{N}$-linked glycosylation sites. Both chains are linked together by five disulphide bonds. Purple dots indicate $\mathrm{N}$-linked glycosylation site cysteine. 
megakaryocytes, a type of bone marrow cell responsible for the production of platelets $^{33.40}$. CLU exists as a heterodimeric structure in alpha and beta subunits, each roughly $40 \mathrm{kDa}$ that are linked by five disulphide bonds (Figure 1.5). Particular regions located within clusterin have been found to have some homology with certain members of the complement system (e.g., C7-9), the chaperone protein ApoA-I, and myosin heavy chain ${ }^{34,35,39,41}$.

CLU is a mammalian protein that has been identified in mouse, rat, quail and human ${ }^{42-48}$. Clusterin is expressed in all tissues of the human body. . Apart from the brain, high levels of clusterin mRNA are found in the stomach, liver, testis, epididymis and ovaries, whereas there are low levels of expression in the breast, lung, heart and spleen. Interestingly, CLU is not expressed in T lymphocytes ${ }^{47}$. It is also found in plasma and cerebrospinal fluid ${ }^{49}$ High expression levels suggest that the function of clusterin is generally important for maintaining homeostatic processes. Within the body, circulating clusterin is incorporated/associated with high-density lipoproteins (HDL) and is bound to ApoA-I. CLU is expressed at fluid-tissue interfaces within tissues, thus clusterin is not always expressed by all cells in the entire organ. This suggests a role in protection of cell membranes by bodily fluids (e.g., protection from bile and gastric juices as well as from other harmful proteins and organelles that exhibit a detrimental effect to healthy tissues) ${ }^{47,49}$.

One unique structural property of clusterin is that it has three separate molten globular domains that enables it to undergo various conformational changes so it can bind proteins in different regions in order to stabilize misfolded proteins $^{50}$, exhibiting chaperone protein properties. This feature allows clusterin to be implicated in many various homeostatic processes in the body such as complement regulation, sperm maturation, apoptosis, lipid transport, promotion of cell-to-cell interactions, membrane protection and endocrine secretion, facilitation of ER stress. Furthermore, CLU is upregulated in several diseases (at mRNA and protein levels). A portion of the functions of clusterin will be discussed below: 


\subsubsection{Reproduction}

CLU was first detected and isolated from rat ram testis fluid in Sertoli cells ${ }^{51}$. CLU is also located in several other regions of the reproductive tract such as in epididymal principal cells and spearmatozoa ${ }^{52}$. In the reproductive system, clusterin serves a protective role in aiding sperm maturation as well as cytoskeletal generation of sperm tails ${ }^{53}$.

\subsubsection{Complement Regulation}

CLU is an element of the membrane attack complex (SC5b-9) and functions as a complement inhibitor ${ }^{34,35,54}$. The activity of clusterin in the complement pathway in the immune system resembles S-protein (also known as vitronectin), which is also part of the SC5b-9, even though the proteins are quite different in structure. When clusterin is bound to $C 5 b-6$, it inhibits the generation of the membrane-attack complex, which in turn inhibits complement mediated cytolysis $^{34,35,54}$.

\subsubsection{Ageing and Oxidative Stress}

ROS (reactive oxygen species) are upregulated during ageing, which contributes to deleterious effects with homeostatic processes ${ }^{55,56}$. CLU exhibits the same upregulating pattern within an ageing organism, which elucidates that clusterin plays a protective role in oxidative injury. Due to the specific regulatory element in clusterin and the AP-I regions located in the gene promotor, clusterin is sensitive to environmental stress such as ionizing radiation ${ }^{57,58}$, heavy metals, proteolytic stress, heat shock stress, and several oxidants.

\subsection{Clusterin and Alzheimer's disease}

In $A D, C L U$ is expressed in high levels in astrocytes and pyramidal neurons in the areas of the brain that are most affected in $A D$, the entorhinal cortex and hippocampus, as well as in senile plaques ${ }^{59-62}$. Many studies have investigated how CLU is implicated in the toxicity, binding and potential clearance of amyloid 
plaques across the BBB via binding to $\mathrm{LRP}^{63}$ in in vitro ${ }^{64-67}$ and in vivo AD models of $A D^{68,69}$. The presence of CLU enhances the clearance of $A \beta 42$ across the BBB by $83 \%$ compared to the absence of the CLU protein ${ }^{70,71}$.

It has also been shown that CLU acts in combination with APOE to aid clearance of amyloid beta plaques in in vitro and in vivo models ${ }^{66,70}$. Observations have also been recently replicated and further investigated from experiments usage of a PDAPP mouse model expressing human APP in conjunction with a particular $A D$ mutation (VF17F) resembling a human $A D$ phenotype ${ }^{70,71}$. APOE levels also seem to directly correlate with levels of CLU, as within an $\mathrm{APOE}^{-/}$PDAPP mouse model, decreased levels of CLU are found compared $\mathrm{APOE}^{+/+}$PDAPP mice ${ }^{70}$.

Research has primarily focused on the relationship between $A \beta 42$ and CLU; however it has recently been suggested that CLU also has quite an important role in associating and clearing $A \beta 40$ species as well. Recent studies have investigated the role of CLU in the aggregation and disaggregation of fibrils that occur in A $\beta 1-40$. Through confocal two-colour coincidence detection (CTCCD) and two-colour version of total reflection fluorescence microscopy (TIRFM) techniques, CLU has been shown to form stable complexes with A $\beta 1-40$ and by the sequestration of $A \beta$ oligomers; $C L U$ impacts the behaviour of $A \beta 1-40^{72}$. Specifically, CLU is predominantly associated with $A \beta 40$ in comparison to $A \beta 42$ plaques as immunoreactivity in $A D$ brains show a stronger ratio of $A \beta 40$ labelling in the cerebral cortex ${ }^{73}$.

Within the body of a healthy individual, clusterin is therefore found in balanced levels that are sufficient to prevent abnormal oligomer growth, in turn protecting the body from oligomer toxicity ${ }^{72}$. The protective properties of CLU have been identified and have been the sole focus of research in discovering a new mechanism that can help shed light on AD progression toxicity ${ }^{72}$. However, it should be noted that there are debates about the beneficial properties of CLU. Previous studies have also claimed to have identified CLU contributing negative affects within $A D^{67-69}$. Regardless of the contribution of CLU to Alzheimer's disease, the role for CLU in the link between cholesterol and autophagy is poorly understood. 


\subsection{Clusterin-mediated Lipid Metabolism}

One of the first known functions of CLU is that it was implicated in cholesterol transport ${ }^{33,74,75}$, as high density lipoproteins (HDL) have been recognised to be involved in reverse cholesterol transport ${ }^{76}$. Clusterin exhibits its apolipoprotein activity by binding to megalin (LRP2) receptors and aiding cholesterol transport.

As clusterin is made up of APOA-I, cholesteryl esters associate with HDL and act synergistically with APOE, it's interesting that even though clusterin also shows similar functionality to APOA-I, it shows having a separate functionality from APOE in terms of cholesterol efflux. This has been demonstrated when CLU regulated cholesterol levels within an APOE knockout mouse model.

In mouse models, clusterin has been shown to inhibit hepatic steatosis by negative regulation of LXR (liver $X$ receptor) and SREBP-1c (sterol regulatory binding protein $1 \mathrm{c}$ ), thus resulting in the inhibition of cholesterol accumulation ${ }^{77}$ Different concentrations of clusterin have also been tested with foam cells expressing high levels of cholesterol and cholesterol esters. Studies have shown that clusterin induces phospholipid efflux, promotes cholesterol efflux and regulates intracellular cholesterol ester levels, therefore showing protective effects and potentially exhibiting antiatherogenic properties ${ }^{78}$.

Interestingly, even though previous literature looks at clusterin and its general function in relation to cholesterol efflux and regulation, no studies have focused on the impact of clusterin on cholesterol metabolism in Alzheimer's disease. Primarily, studies have instead focused on obvious cholesterol implicated diseases such as atherosclerosis ${ }^{148}$. With levels of cholesterol and other lipids being a key regulator of many processes in the brain, it is apparent that disruption to normal levels of cholesterol would result in impairment of the normal functioning of the human brain which would lead to various negative secondary effects. Further investigations into CLU-mediated cholesterol metabolism in the brain are key to understanding the pathology of AD. 


\subsection{Clusterin-mediated Autophagy}

CLU and autophagy are independently associated with tumour suppression in the first stages of carcinogenesis, resistance to anti-cancer treatments in later stages, increased aggreopathies and reduced protein homeostasis ${ }^{1,27}$. The role of CLU in autophagy has been poorly understudied until it was recently discovered that CLU enhances the survival of cancer through aiding the lipidation of LC3I to LC3II, as well as promoting the stability of LC3Atg3 binding complex ${ }^{79}$. This results in autophagy activation and upregulated production of autophagosome formation. Therefore, CLU aids survival of cells via an autophagy-dependant mechanism. Since CLU is highly expressed when anticancer treatments are administered, silencing CLU could prove to be a beneficial treatment towards tackling tumorigenesis ${ }^{79}$ Further insights into how CLU functions in combination with other cellular pathways will prove to be beneficial in how it affects the homeostasis of other genes in neurodegenerative diseases.

\subsection{Model Organisms to Investigate Alzheimer's Disease}

\subsubsection{SH-SY5Y cell model}

Using a neuroblastoma cell line derived from the SK-N-SH cell line, SHSY5Y cells have many distinguishing functional and biochemical features representative of neurons $s^{80}$. These cells also have the potential to be induced to differentiate into having dendrite-like morphology when given various treatments such as brain-derived neurotrophic factor (BDNF), TPA or retinoic acid $^{81-84}$. This has enabled scientists to adopt SH-SY5Y cells as a popular neuronal cell model to investigate changes and effects on AD phenotypes, especially when analysing A $\mathrm{B} 1-42$ pathology. SH-SY5Y cells are also used for neurotoxic research, as when SH-SY5Y cells are subjected to cytotoxic compounds, the cells mimic responses which are found in human primary cultures ${ }^{85}$

Throughout this thesis, the AD cell model used in experiments will be SHSY5Y cells expressing the Swedish mutation form of APP (swAPP $\left.{ }_{695}\right)$. In $A D$, three 
isoforms of $\mathrm{APP}$ are generated; $\mathrm{APP}_{695}, \mathrm{APP}_{751}$ and $\mathrm{APP}_{770}$, with $\mathrm{APP}_{695}$ being highly expressed in the brain in comparison to $\mathrm{APP}_{751}$ and $\mathrm{APP}_{770}{ }^{86}$. However, $\mathrm{APP}_{695}$ is the only isoform which seems to correlate to high levels $A \beta 40$ and $A \beta 42$ generation along with the increase of the APP intracellular domain (AICD) which regulates expression of genes such as neprilysin (NEP), an A $\beta$-degrading enzyme ${ }^{87}$ Furthermore, when a Swedish mutation of $\mathrm{APP}_{695}$ is expressed, the pathology is markedly increased compared to wild type APP ${ }_{695} \mathrm{SH}-\mathrm{SY} 5 \mathrm{Y}$ cells ${ }^{87}$. This increase is due to the double mutation found at lysine and asparagine at residue 595 and methionine to leucine mutation at position 596. The latter mutation is primarily responsible for the accumulation of plaque pathology ${ }^{88-90}$.

While this cell line is extensively used to investigate neurodegenerative diseases, it should be noted that SH-SY5Y neuronal like activity such as expressing dopaminergic markers and its adrenergic phenotype decrease with increasing passage numbers. Therefore, it is advised that passage number of cells should be kept as low as possible and ideally should not exceed beyond passage twenty (P20). If cells are used beyond P20, neuronal markers should be used to insure the required behaviour is still present within the cell line. Several studies have used SH-SY5Y cells to investigate AD. However, no studies to date have used this cell line to investigate the effects of clusterin in AD. Utilizing this model for an in vitro model would be beneficial in complementing research from in vivo $A D$ experiments.

\subsubsection{Fruit fly Model of Alzheimer's disease}

Drosophila melanogaster, a species of fruit fly that is a model organism for many human diseases, was one of the first model organisms to have its genome fully sequenced ${ }^{91}$. This organism has complex structural anatomy and a long history of being exploited for studying mechanisms and pathways of learning and memory ${ }^{92,93}$. As $D$. melanogaster has been used as one of the earliest model organisms utilised in the science industry; many tools have been developed over the years in order to optimise different research approaches using this organism ${ }^{94}$. Having a short lifespan of 120 days, accumulation of data is easy to obtain and is good for looking at ageing process along with age-related 
neurodegenerative diseases such as Parkinson's disease and Alzheimer's disease.

As $70 \%$ of human disease-causing genes have orthologues in D. melanogaster, processes in relation to human activities and processes can be linked through this model organism ${ }^{95}$.

Relevant to $A D$, the $D$. melanogaster genome contains all sections of the gamma-secretase complex ${ }^{96}$ as well as the Drosophila homolog of human APP $(d A P P L)^{97} . d A P P L$ share many similar characteristics with the APP gene family in

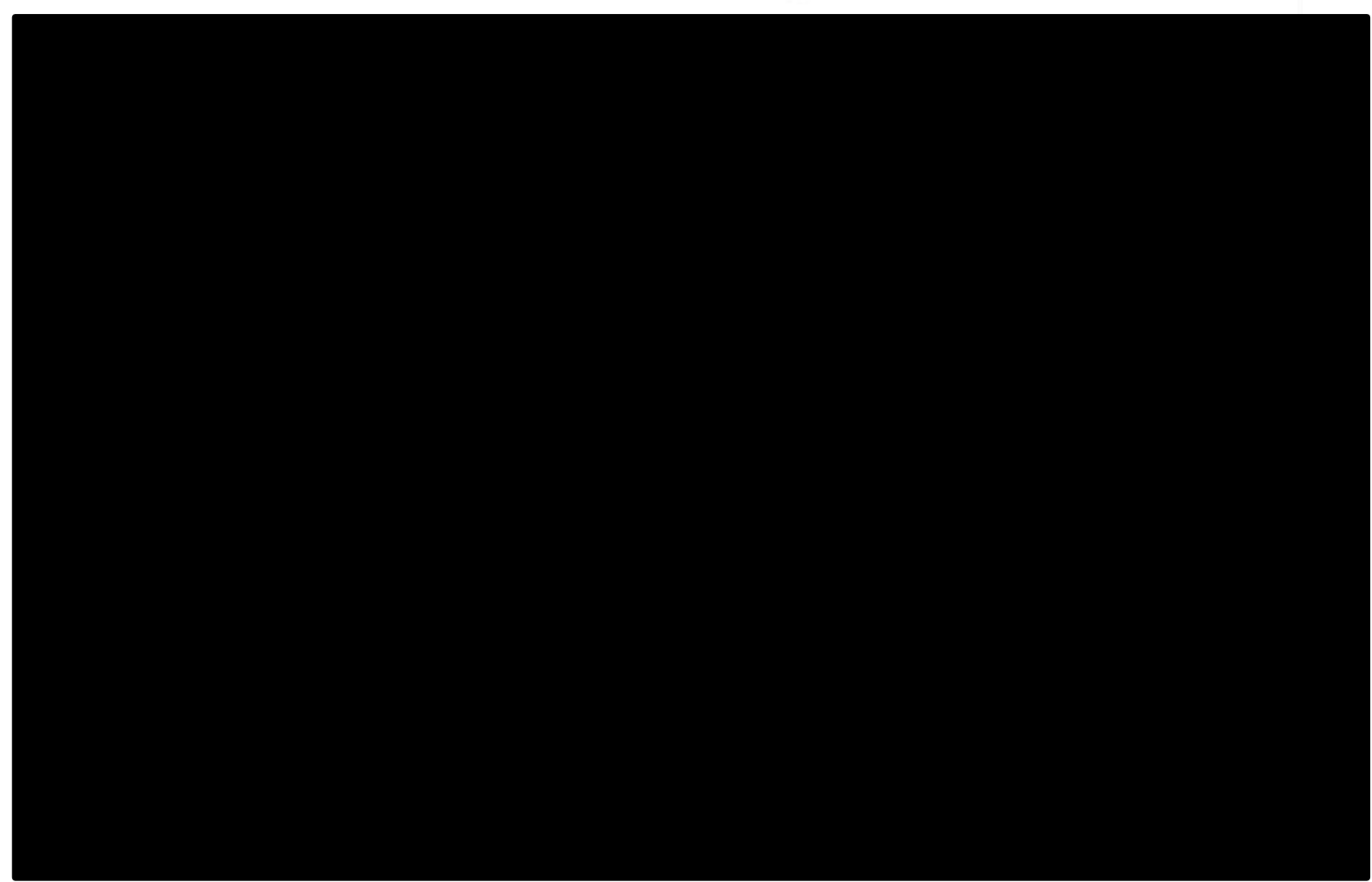

Figure 1.6: The GAL4/UAS (upstream activating sequence) system in D. melanogaster Figure adapted from Miratu et al, 2002. To induce abnormal gene expression within Drosophila in either a particular area of interest or systemically, the GAL4/UAS system is utilised. The human gene of interest is placed downstream of the UAS region that is constituted of several sites that GAL4 can bind to. The yeast transcriptional activator (GAL4 driver) is expressed in another fly line which most likely will be placed downstream of a promotor specific for a cell or tissue section such as ELAV (embryonic lethal abnormal vision) (Berger et al, 2007). With both parts of the system being isolated within both flies, both sections of the system are essentially inactive. However once both transgenic fly lines are crossed over, GAL4 binds to the UAS region and transcribes the human gene of interest.

vertebrates, however they do not contain an $A \beta 42$ cleavage site. Therefore, in order to investigate the effects of Alzheimer's disease within this organism, transgenic flies expressing human APP and BACE (requirements to generate A $\beta 42$ ) have been constructed through using the UAS/GAL4 system (Figure 1.6) ${ }^{98,99}$. 
Previous studies have upregulated the expression of Tau and subsequent production of $A \beta 42$ in flies; these studies show phenotypic effects in neuronal decline such as decreased locomotion in both larvae and adult flies, diminished lifespan, hindrance in flight ability, and deformities in eye texture ${ }^{98}$. An additional bonus to working with these phenotypes is that these phenotypes can be quantified, thus generating a reliable output of information. Cognitive defects, a noted symptom of AD patients, have also been observed in AD flies.

Relevant to this thesis, it is critical to note that $D$. melanogaster does not contain CLU. This is actually experimentally advantageous as demonstrated by a previous ageing study that expressed human CLU in D. melanogaster without having to be concerned with any effects of endogenous $\mathrm{CLU}^{100}$. In that study, human CLU extended the lifespan of $D$. melanogaster as well as increased tolerance to oxidative stress, starvation, and heat shock. ROS (reactive oxygen species) levels were also decreased among other protective activities ${ }^{100}$. It was thus proposed that CLU acts as an antioxidant protein and also that the disulphide linkages and sulfydryl groups of cysteines in CLU interact with misfolded proteins. When these groups were inactivated by addition of $\mathrm{N}$ ethylmaleimide, the protective role of clusterin vanished ${ }^{100}$. While the role of CLU has been examined in mouse models of Alzheimer's disease ${ }^{101}$, studies have not yet looked at how CLU functions in a fly model of AD. 


\subsection{AIMS AND HYPOTHESES}

It is seen in previous reports that CLU has been associated with several processes defective in the pathology of Alzheimer's disease such as amyloid plaque build-up, cholesterol, and autophagy. However, there has not yet been a focused investigation into mechanisms through which all three components have been linked together. Is AD pathology upregulated purely due to protein burden exhibited within cells that in turn have an effect on $A \beta$ accumulation? Or does the CLU pathway operate separately to $A \beta 42$ in relation to cholesterol and increased autophagy?

From information gathered from previous studies, I hypothesize that increasing CLU expression will reverse certain pathological aspects of Alzheimer's disease and lead to a reduction in A $\beta$ levels, decrease levels of APP, increase efflux of cholesterol, upregulate cholesterol transport and upregulate $A \beta_{40}$ secretion in the brain. To test this hypothesis, I will investigate these processes in vitro (SH-SY5Y cell line) and in vivo (Drosophila melanogaster) models of AD. If observations match the proposed hypothesis, then this study would be a powerful case to consider in regards to the therapeutic potential of CLU to treat patients who suffer from $A D$ and perhaps other forms of dementia. 


\section{CHAPTER 2}

\subsection{INTRODUCTION}

Alzheimer's disease $(A D)$ is a progressive detrimental brain disorder that is characterised primarily by the irreversible deterioration of the brain leading to increased impairment of language function and memory ${ }^{3}$. First manifesting as dementia within elderly patients, there is currently no cure or effective therapy to treat $A D^{153}$. Genetic risk factors by GWAS studies include APOE, APOJ (CLU), CR1 and PICALM. The top two genetic risk factors, APOE and APOJ (CLU), are known to both be involved in cholesterol homeostasis. APOE and CLU are similar in their functionality as both proteins have been demonstrated to cooperatively bind and clear excess cholesterol within the brain, as they have the ability to bind cholesterol and pass through the blood brain barrier (BBB) so cholesterol can be utilized by other mechanisms in the body ${ }^{40}$. Much research has been invested within examining APOE, as it's been thought of as the key player in developing a therapeutic treatment towards patients; however no treatments from this research have emerged. Less research has been directed into CLU functionality and the roles that this gene plays within an Alzheimer's disease model. Despite previous research, it remains unresolved whether the overexpression of CLU results in a therapeutic or detrimental effect within treating the AD condition.

$\mathrm{CLU}$ is an $80 \mathrm{kDa}$ linked glycoprotein ${ }^{39}$ which is located on chromosome $8 p 21^{40}$ which is structured into nine axons ${ }^{41}$ and is synthesised by megakaryocytes. Even though there is not a complete understanding of the function of $\mathrm{CLU}$ in $A D^{62,63}$, other roles within the mammalian system have been identified. This includes CLU being involved in complement regulation $34,35,54$, reproduction $^{52,53}$, as well as acting as in a similar fashion to heat shock proteins, with CLU production induced under stress, and serving protective roles by binding misfolded proteins and organelles in attempts to stabilize them ${ }^{39}$.

One of the recent functionalities of CLU that has been identified is its involvement in autophagy in a cancer cell line, as upon administration of CLU in PC3 prostate cancer cell model; CLU aids the lipidation processes of LC3, as well 
as stabilizing the LC3-Atg3 complex. This results in correct autophagosome formation as well as correct induction of autophagic processes which in turn enhances cancer survival and incurs resistance towards anti-cancer treatments. If CLU responds in the same manner within an Alzheimer's model organism, there is potential for CLU to protect and aid the survival of neurons, which in turn should decrease deterioration of the brain.

Given that CLU is involved in cholesterol and autophagic processes, this suggests that these two pathways are linked. It makes sense to examine these two processes and CLU in how they interact in regards to processing APP and clearance of $A \beta_{42}$. To date, there has not been a determined relationship or mechanism through which all three components have been linked together. Is $A D$ pathology upregulated purely due to protein burden exhibited within cells which in turn have an effect on amyloid beta accumulation? Or does the pathway operate separately to $A \beta_{42}$ in relation to cholesterol and increased autophagy? From the protective roles of CLU, it can be hypothesised that increasing CLU expression will reverse certain pathological aspects of Alzheimer's disease and lead to a reduction in A levels; decrease levels of APP, increase efflux of cholesterol and up regulate cholesterol transport in the brain. If observations match the proposed hypothesis, then this study would be a powerful case to consider in regards to the therapeutic potential of targeting CLU in patients who suffer from $A D$ and perhaps other forms of dementia.

Here I hypothesise that increasing CLU expression will reverse certain pathological aspects of Alzheimer's disease and lead to a reduction in $A \beta$ levels, decrease levels of APP, increase efflux of cholesterol and up regulate cholesterol transport. To evaluate this hypothesis, cholesterol metabolism, autophagy regulation, APP processing and $A \beta_{42}$ clearance will be examined in vitro (SH-SY5Y cell line) and in vivo (Drosophila melanogaster) Alzheimer's disease (AD) models. Levels of APP, amyloid beta, cholesterol and autophagy levels will be measured and then compared between in vitro and in vivo $A D$ models that have overexpressed levels of clusterin. This will be done by administration of a clusterin containing plasmid in each model. This will allow for the determination of the effects of CLU in whether it promotes or reverses the pathology of AD. 


\subsection{METHODS}

\subsubsection{Maintenance of Organisms}

Cells: SH-SY5Y and SH-SY5Y SWAPP cells were obtained from Dr Jerry Turnbull (University of Liverpool) and grown in RPMI medium $+10 \%$ FCS $+1 \%$ penicillin streptomycin. Cells were split every $2-4$ days, seeded at $1 \times 10^{\wedge 5}$ cells/mL. Growth of APP cells was approximately double the speed of WT cells. Transfected cells were incubated without the presence of antibiotics.

Fruit Flies: Lines of Drosophila melanogaster were grown and maintained at $22^{\circ} \mathrm{C}$. Food material (for $500 \mathrm{~mL}$ ) consisted of $5 \mathrm{~g}$ agar, $20 \mathrm{~g}$ yeast, $55 \mathrm{~g}$ ground cornmeal and $500 \mathrm{~mL}$ MilliQ H20. Ingredients were brought to a boil and simmered for $2 \mathrm{~min}$, and then $10 \mathrm{ml}$ molasses, $65 \mathrm{~g}$ sugar, $1.6 \mathrm{~g}$ Moldex and 18 $\mathrm{mL} 95 \%$ ethanol were mixed with the solution. The fly model of Alzheimer's disease (APP/BACE) was obtained from the Bloomington Stock Centre. The wild type fly (wt188) was used to cross all fly genotypes analysed (WT, APP/BACE, APP/BACE/CLU and ELAV/CLU) to the same genetic background after CLU overexpressing flies were generated.

\subsubsection{Expression of Clusterin}

SH-SY5Y cells: To express CLU in SH-SY5Y cells, the mammalian expression vector pcDNA3.1(+) and pUAST-attb + CLU (pUAST-attB vector from Groth et al, Stanford University ${ }^{154}$ were digested with Notl and Xbal restriction enzymes for $1 \mathrm{hr}$ at $37^{\circ} \mathrm{C}$ and then treated with Calf Intestine Phosphatase (CIP) for an additional $1 \mathrm{hr}$ at $37^{\circ} \mathrm{C}$. Products were electrophoresed on a $0.7 \%$ agarose gel, and purified using a gel purification kit (Geneaid) according to the manufacturer's instructions. Ligation following band excision was carried out overnight for $16 \mathrm{hrs}$ at $4^{\circ} \mathrm{C}$ and consisted of the following: $10 \mu \mathrm{L} \mathrm{CLU}$ Insert (1373bp), $2 \mu \mathrm{L}$ pcDNA3.1(+) vector (5416bp), $2 \mu \mathrm{L}$ 10X buffer, $4 \mu \mathrm{L} \mathrm{dH}_{2} \mathrm{O}$ and $2 \mu \mathrm{L}$ T4 DNA ligase (Invitrogen). 
Reporter construct GFP addition to CLU vector: To verify ligation and quantify efficiency of CLU transfection into SH-SY5Y cell lines, we introduced a GFP sequence within pcDNA3.1(+). The 705 bp GFP segment from vector RG203629 (Origene) was PCR amplified using primers that were $59 \mathrm{bp}$ and $29 \mathrm{bp}$ long that contained $18 \mathrm{bp}$ and $13 \mathrm{bp}$ of the original GFP sequence (Table 2). The

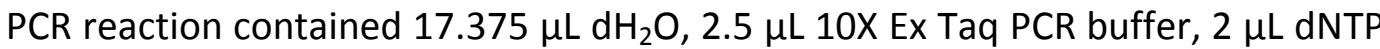
(2.5mM), $1 \mu \mathrm{L}$ forward primer $(10 \mu \mathrm{M}) 1 \mu \mathrm{L}$ reverse primer(10 $\mu \mathrm{M}) 0.125 \mu \mathrm{L}$ Ex Taq (Takara), and $2 \mu \mathrm{L}$ RG203629 plasmid DNA containing GFP. PCR was carried out in the Techne TC 5000 PCR machine with the following parameters: $94^{\circ} \mathrm{C}$ for $10 \mathrm{~min}$, followed by $36 \mathrm{cycles}$ of $94^{\circ} \mathrm{C}$ for $30 \mathrm{sec}, 51^{\circ} \mathrm{C}$ for $30 \mathrm{sec}$ and $72^{\circ} \mathrm{C}$ for $2 \mathrm{~min}$, and a final extension at $72^{\circ} \mathrm{C}$ for $10 \mathrm{~min}$. PCR products were purified using the Purelink ${ }^{\circledast}$ Quick Plasmid Miniprep Kit (Invitrogen) according to the manufacturer's instructions.

pcDNA3.1(+) + CLU + GFP transfection: To overexpress clusterin in SY5Y cells, we transfected a plasmid expression vector (pcDNA3.1(+) + CLU + GFP) using Lipofectamine 2000 (Invitrogen) Briefly, cells were grown to $80 \%$ confluency, $4 \mu \mathrm{L}$ Lipofectamine ${ }^{\circledR} 2000$ reagent was diluted in $100 \mu \mathrm{L}$ RPMI medium, and $2 \mu \mathrm{g}$ DNA was diluted in $500 \mu \mathrm{L}$ RPMI medium. Transfection reagent and DNA were added in a 1:1 ratio and incubated for $5 \mathrm{~min}$ at room temperature. The DNA-lipofectamine complex (1:2) was added to SH-SY5Y cells and incubated for $4 \mathrm{hrs}$ before replacing transfection media with normal growth media. Cells were further incubated for 1-2 days. Transfected cells were identified using the GFP filter (FITC) on the fluorescent microscope.

\begin{tabular}{|c|c|}
\hline Primer & Sequence \\
\hline Forward & $\begin{array}{l}\text { 5'CCTCTAGACCGAGGAGATCTGCCGCCGCGATCGCCGGCGCGCTCGAGATGGAGAGCGAC3' } \\
\begin{array}{cc}\text { Xbal site } & \text { RBS and Kozac sequences site and start of GFP }\end{array}\end{array}$ \\
\hline Reverse & $\begin{array}{c}\text { 5' CCCGTTTAAACTTAAACTCTTTCTTCACC 3' } \\
\text { Pmel site GFP end sequence }\end{array}$ \\
\hline
\end{tabular}

Table 1: RG203629 primer sequences 
Fruit flies: Human CLU was expressed in D. melanogaster with a series of steps culminating in microinjection of a PCR construct. First, a plasmid containing human CLU was purchased (DNASU Plasmid Repository, Cat\# HsCD00000239); this plasmid contained a mutation at 123 bp with an L6R substitution. To correct this mutation and restore WT version of CLU, the plasmid was digested with Sall and Xbal restriction enymes and the 625 bp fragment was PCR amplified using primers that contained $28 \mathrm{bp}$ and $15 \mathrm{bp}$ flanking regions of the pDNR-Dual vector (Table 1) which had the desired cloning sites for CLU to be excised and ligated into the fly vector pUAST-attB. Within the primer, amino acids CTG were added to correct the amino acidsCGG (arginine). The PCR reaction was carried out as follows: Mix 1 (for three reactions): $3 \mu \mathrm{L}$ dNTP $\operatorname{mix} 2.5 \mathrm{mM}$ ), $4.5 \mu \mathrm{L}$ forward primer $(10 \mu \mathrm{M}) 4.5 \mu \mathrm{L}$ reverse primer $(10 \mu \mathrm{M})$ and $61.5 \mu \mathrm{L} d \mathrm{H} 20$; Mix 2 (for 3 reactions): $57.75 \mu \mathrm{L} d H 20,15 \mu \mathrm{L} 10 x$ Ex Taq PCR buffer $2.25 \mu \mathrm{L}$ Expand Polymerase (Sigma-Alrich). The template (pDNR-Dual + CLU) for PCR reaction was $0.5 \mu \mathrm{L}$ of forward and reverse primers, $24.5 \mu \mathrm{L}$ 'Mix 1' and 25.5 $\mu \mathrm{L}$ 'Mix 2'. Cycling parameters for PCR amplification on the Techne TC 5000 PCR machine were the following: $94^{\circ} \mathrm{C}$ for 2 min, 3 cycles of $94^{\circ} \mathrm{C}$ for $15 \mathrm{sec}, 55^{\circ} \mathrm{C}$ for $30 \mathrm{sec}$ and $72^{\circ} \mathrm{C}$ for $45 \mathrm{sec}, 32 \mathrm{cycles}$ of $94^{\circ} \mathrm{C}$ for $15 \mathrm{sec}, 63^{\circ} \mathrm{C}$ for $30 \mathrm{sec}$ and $72^{\circ} \mathrm{C}$ for $45 \mathrm{sec}$, and a final extension of $72^{\circ} \mathrm{C}$ for $7 \mathrm{~min}$. The resulting PCR product and pDNR-Dual vector were each digested with Sall and Smal, gel purified and ligated with a 5 min ligation using the Quick Ligation Kit (New England Biolabs). The ligated product of a PCR-corrected and now WT version of human CLU in pDNR-Dual vector was transformed into competent E.coli cells and purified using purification kit (Invitrogen). To insert DNA into fly embryos via microinjection, we introduced human CLU in a specialised vector used for microinjection (i.e., pUAST-attB). pDNR-Dual + CLU vector was digested with Sall and Xbal, and pUAST-attB was digested with Xhol and Xbal. Insert and vector were CIP treated, purified, ligated and transformed into competent E. coli. The resulting plasmid of human CLU in pUAST-attB was purified using the PureLink ${ }^{\circledR}$ HiPure Plasmid Miniprep Kit (Life Technologies) according to the manufacturer's instructions.

In order to deliver CLU DNA into flies, this is achieved by microinjecting DNA into fly embryos. To microinject the plasmid containing human CLU into 
flies, an aliquot of the pUAST-attB + CLU plasmid was concentrated by precipitation. $10 \mu \mathrm{L} 3 \mathrm{M} \mathrm{NaAc}(\mathrm{pH} 5.2)$ and $220 \mu \mathrm{L} 100 \%$ ethanol were added to $20 \mu \mathrm{g}$ of DNA in $100 \mu \mathrm{L} \mathrm{dH} 2 \mathrm{O}$, incubated at $-20^{\circ} \mathrm{C}$ overnight, and centrifuged at $13,000 \times \mathrm{g}$ for $10 \mathrm{~min} \mathrm{~s}$ at $4^{\circ} \mathrm{C}$. Pellets were then washed with $70 \%$ ethanol and resuspended in $50 \mu \mathrm{L}$ microinjection buffer $(50 \mathrm{mM} \mathrm{KCL}, 0.1 \mathrm{mM}$ sodium phosphate) as previously described by ${ }^{102}$.

The microinjection is the final step to directly getting the CLU DNA within the fly by injecting the eggs of the fly at a mid-interval developed stage. Overdeveloped eggs are discarded, as CLU will not be efficiently taken up the egg. The microinjection procedure began 2-3 days prior to injection when, extra yeast was added to the food of mature, 3-4 week old wild type flies (strain WT118). The day before injection, approximately 20-30 egg plates were made. On the morning prior to injection, a small amount of yeast paste (water and yeast) was placed in the centre of the egg plates. Paste on the egg plate serves as an adhesive material for laying of eggs by flies, so these can be collected and used for dechorionation and microinjection. Wild type flies were then transferred to a plastic chamber and sealed with the egg plate. To induce laying of eggs, flies were incubated at $25^{\circ} \mathrm{C}$ for $1 \mathrm{hr}$. Purified DNA was also prepared for injection by centrifugation at $12,000 \times \mathrm{g}$ at $4^{\circ} \mathrm{C}$ for $10 \mathrm{~min}$.

Embryos were collected and egg plates were switched at an average rate of $25 \mathrm{~min}$ (in order to collect the maximal amount of eggs laid by flies), transferred to a prepared microscope slide with $10 \mathrm{~mm} \times 20 \mathrm{~mm}$ Scotch adhesive tape with a fine point paint brush, dechorionated via. blunt forceps for 5-20 min, then transferred and organised in rows of 12-24 eggs on a $5 \mathrm{~mm} \times 10 \mathrm{~mm}$ Scotch adhesive tape adjacent to the site where the dechorionation was executed. Dechorionation is carried out to break the outer capsule of the egg and expose the embryo for microinjection. 1-2 drops of Halocarbon Oil 700 (Life Technologies) was placed over dechlorionated eggs and was dehydrated using silica gel for 10-15 min. An Eppendorf FemtoJet Express with a micromanipulator mounted on an Olympus CK2 Inverted Microscope was used for microinjection of precipitated DNA. Surviving pupae were directly placed in an air tight plastic 
vessel lined with damp towels and incubated at $18^{\circ} \mathrm{C}$ for 1 day, and then provided with a slight oxygen burst and further incubated at $22^{\circ} \mathrm{C}$ for 1 day.

\begin{tabular}{|l|l|}
\hline Primer & Sequence \\
\hline Forward & $\begin{array}{r}\text { 5' ATAATAGTCGACGGATCCACCATGATGAAGACTCTGCTGCTGTTTG 3' } \\
\text { Sall and BamHI siter start of pDNR-Dual }\end{array}$ \\
\hline Reverse & $\begin{array}{r}5^{\prime} \text { TAAGCCTAATAACCCGGGTGAAGAACCTGTCCT 3' } \\
\text { Smal site pDNR-Dual end sequence }\end{array}$ \\
\hline
\end{tabular}

Table 2: pDNR-Dual primer sequence

\subsubsection{Protein Extraction}

Cells: For Western Blot analysis, cells were grown in 12 well dishes to $\sim 80 \%$ confluence and then transfected for $20 \mathrm{~h}$ with the constructed clusterin plasmid. Then $1 \mathrm{~mL}$ of PBS was added to each well after media was removed and left on ice for $1 \mathrm{~min}$, PBS was removed and $50 \mu \mathrm{L}$ Protease Inhibitor (Roche) dissolved in RIPA buffer (Sigma) was added to each well and left for $5 \mathrm{~min}$. Cells were harvested at $4^{\circ} \mathrm{C}$, placed in a $1.5 \mathrm{~mL}$ microcentrifuge tube, vortexed for 30 min at 5 min intervals, centrifuged at $13,000 \mathrm{~g}$ at $4{ }^{\circ} \mathrm{C}$, and supernatant was transferred to a fresh 1.5 microcentrifuge tube and stored at $-80^{\circ} \mathrm{C}$.

For ELISA cells were grown in $100 \mu \mathrm{L}$ of media (RPMI $+10 \%$ FCS) in 12 well plates and transferred to a $1.5 \mathrm{~mL}$ microcentrifuge tube with HALT protease inhibitor centrifuged for $5 \mathrm{~min}$ at $4^{\circ} \mathrm{C}$ at $13,000 \times$ g. Samples were stored at $-80^{\circ} \mathrm{C}$. Protocol for specific detection of $A \beta 42$ was implemented as outlined in the manufacturer's instructions.

Fruit Flies: For Western Blot analysis, $\sim 50$ fly heads were collected and instantly lysed in $100 \mu \mathrm{L}$ RIPA buffer containing Protease Inhibitor Cocktail (Roche) using a motorised pestle for $1 \mathrm{~min}$ with $20 \mathrm{sec}$ stroke intervals whilst being kept continuously on ice. Lysate was centrifuged for 2 min at 13,000 g at $4^{\circ} \mathrm{C}$ and supernatant was transferred to a fresh $1.5 \mathrm{~mL}$ microcentrifuge tube. Protein for all mentioned extractions was quantified using the Pierce BCA Protein Assay Kit (Thermo Scientific) according to the manufacturer's instructions. 


\subsubsection{Western Blot Analysis}

Cells and fruit flies: Protein extracted from cells and flies was investigated via Western blot analyses. $20 \mu \mathrm{g}$ of protein was electrophoresed with the addition of $6 \mu$ l beta-mercaptoethanol and $12 \mu \mathrm{l}$ 5X SDS loading buffer (for $5 x$ stock: $250 \mathrm{mM}$ Tris $\mathrm{HCl} \mathrm{pH} \mathrm{6.8,} \mathrm{10 \%} \mathrm{SDS,} \mathrm{30 \%} \mathrm{glycerol,} \mathrm{0.02 \%} \mathrm{bromophenol} \mathrm{blue).}$ For SDS-PAGE, equal amounts of protein were run on a $7.5 \%$ Tris-bis gel (with $4 \%$ stacking gel) for duration of $1.5 \mathrm{~h}$ at $120 \mathrm{~V}$ at room temperature. Proteins were transferred to a $0.2 \mu \mathrm{M}$ PVDF membrane that was normalised with methanol for $90 \mathrm{sec}$ before being rinsed with transfer buffer and transferred using a wet transfer apparatus (Bio-Rad) for $2 \mathrm{~h}$ at $100 \mathrm{~V}$ at room temperature. The transfer buffer recipe for $1 \mathrm{~L}$ was the following: $14.4 \mathrm{~g}$ glycine, $3.03 \mathrm{~g}$ Tris Base and $20 \%$ methanol. The membrane was blocked with $3 \%$ BSA in PBST for $1 \mathrm{~h}$ at $4^{\circ} \mathrm{C}$ followed by incubation of APP polyclonal antibody (Anti-Amyloid Precursor protein, C-Terminal (751-770), cat \# 171610, Calbiochem) at a 1:1000 dilution overnight at $4^{\circ} \mathrm{C}$ for $12-16 \mathrm{hrs}$. The membrane was washed with PBST three times followed by secondary anti-rabbit antibody incubation (1:2000) for $1 \mathrm{~h}$ at $4^{\circ} \mathrm{C}$. The membrane was washed again with PBST three times and visualized with a fluorescent image analyzer (Fujifilm FLA-5100) with $\mathrm{CH} 2$ (Cy5 channel) at 400V. Quantification of FL-APP or CTF-APP was normalised to alpha tubulin densitometry (1:1000 dilution) (alpha-tubulin, cat\#ab18251, Abcam) that was visualised with anti-rabbit secondary (ECL Plex goat-anti rabbit IgG, Cy5, cat \# PA45011, GE Healthcare, VWR Global) used at 1:2000 dilution.

\subsubsection{Cholesterol Localization}

Cells: SH-SY5Y cells were grown in $1 \mathrm{~mL} \mathrm{RPMI}+10 \%$ FCS media on nitric acid-treated coverslips in 12 well plates to $70-80 \%$ confluency. Transfected cells were grown on nitric acid-treated coverslips with the addition of poly-L-lysine $(100 \mu \mathrm{g} / \mathrm{mL})$ and laminin $(50 \mu \mathrm{g} / \mathrm{mL})$ adhesive (Sigma). After $500 \mu \mathrm{L}$ media was removed, $500 \mu \mathrm{L} 4 \%$ PFA $+3 \%$ sucrose was added and incubated for $20 \mathrm{~min}$, followed by a wash with $3 X$ PBS for 5 min prior to the addition of $1.5 \mathrm{mg} / \mathrm{mL}$ glycine for $10 \mathrm{~min}$. After a brief wash for $1 \mathrm{~min}$ with PBS, $1 \mathrm{~mL}$ of $50 \mathrm{mg} / \mathrm{mL}$ Filipin (Sigma, ca\#f767-MG) in DMSO was added and incubated for $2 \mathrm{~h}$. After a 
wash with 3 X PBS, coverslips were mounted on slides with Vectashield antifade mounting medium (Vector Laboratories) and imaged with a 60X objective under the Olympus BX63 fluorescent microscope using the DAPI filter.

Flies: Filipin was also used to visualize cholesterol in brains dissected from flies. Flies were prefixed in PFAT-DMSO (4\% paraformaldehyde in 1x PBS, 0.1\% Triton $\mathrm{X}-100,5 \% \mathrm{DMSO}$ ) for $2 \mathrm{~h}$ rotating at room temperature and washed with PBT (1x PBS, 0.5\% Triton X-100) three times at 5 min intervals. Flies were then transferred and dissected for 10-15 min in PBT in a mini petri dish under a dissecting microscope with forceps. Brains were transferred with a pasteur pipette to cold PTB in a $1.5 \mathrm{~mL}$ microcentrifuge tube, and stored on ice. PBT was then replaced with $368 \mu \mathrm{L}$ fresh PBT solution, $32 \mu \mathrm{L}$ of $38 \%$ formaldehyde and $500 \mu \mathrm{L}$ heptane. Samples were then shaken by hand for $30-45 \mathrm{sec}$, followed by incubation at room temperature to allow the foam to settle, and the upper heptane phase and most of the aqueous phase was removed and transferred to a clean $1.5 \mu \mathrm{L}$ microcentrifuge tube. $700 \mu \mathrm{L}$ of fresh PBT, $63 \mu \mathrm{L}$ of $38 \%$ formaldehyde and $40 \mu \mathrm{L}$ of DMSO was added and incubated on a rocking platform for $20 \mathrm{~min}$ to fix the brains. Fixed brains were washed twice for $5 \mathrm{~min}$ in $100 \%$ methanol and then stored at $-20^{\circ} \mathrm{C}$ in $100 \%$ methanol.

Samples were rehydrated in $1 \mathrm{~mL}$ of $1: 150 \%$ methanol/PBT, each for 5 min to slowly rehydrate the sample, and then washed with $4 \mathrm{X}$ PBT at 5 min intervals to ensure removal of methanol. Then $50 \mathrm{mg} / \mathrm{mL}$ (1:500 dilution in PBT) of filipin (Sigma-Aldrich, cat\# F4767-1MG) was added, incubated for $1 \mathrm{~h}$, and washed four times with $3 \mathrm{X}$ PBT for 5 min intervals. After the final wash was discarded, two drops of Vectashield antifade mounting medium (Vector Laboratories) was added, mounted on a premade bridge slide to avoid flattening of tissue sample, and imaged with a 40X objective under the fluorescent microscope using the DAPI filter on the Olympus BX63 fluorescence upright microscope. 


\subsubsection{Lipid Quantification}

Cells and Fruit Flies: Lipids were extracted from cells and fruit flies using a modification of the Bligh and Dyer method $(1959)^{103}$ with modifications outlined by Guan et al $(2014)^{104}$. Cells were grown to $90 \%$ confluence in 12 well plates, and collected with a cell scraper (McFarlane), and resulting lysate was transferred to a $1.5 \mathrm{~mL}$ microcentrifuge tube for lipid extraction. In contrast, 100 fly heads were homogenised with a handheld motorised pestle (Sigma Aldrich, cat\# Z359971-1EA) for $30 \mathrm{sec}$ in $200 \mu \mathrm{L}$ PBS buffer on ice, and that was used for lipid extraction. For both cells and flies, $600 \mu \mathrm{L}$ of $1: 2(\mathrm{v} / \mathrm{v})$ chloroform:methanol was added to the sample, vortexed for $1 \mathrm{~min}$, and shaken in a Thermoshaker (Ependorf) at $10,000 \mathrm{rpm}$ at $4^{\circ} \mathrm{C}$ for $2 \mathrm{~h}$. Then $230 \mu \mathrm{L}$ water and $200 \mu \mathrm{L}$ chloroform were added and vortexed for $1 \mathrm{~min}$. For phase separation, sample was centrifuged at 10,000 rpm for $2 \mathrm{~min}$, and the lower organic phase was transferred to a chilled $1.5 \mu \mathrm{L}$ microcentrifuge tube. Phase separation was repeated with the addition of $400 \mu \mathrm{L}$ chloroform to the upper phase and centrifugation at 10,000 rpm for $2 \mathrm{~min}$, with the second lower organic phase being pooled with the first lower organic phase. Lower phases were dried with a Centrivap Concentrator (Labconco), and the dried lipid pellet was resuspended in $40 \mu \mathrm{L}$ choloform:methanol (1:1 v/v).

Lipids in the lipid extracts were quantified using Matrix-assisted laser desorption/ionisation-time of flight (MALDI-TOF) mass spectrometer. The matrix 2, 5 dihydroxybenzoic acid (DHB) was selected and was prepared with acetonitrile: ddH2O (1:1) with the addition of $1 \%$ trifluroacetic acid (TFA) and 10 mg DHB. A dilution of sample:matrix (1:100) was spotted on a 384 Opti-TOF 123x81 mmRevA MALDI plate and analysed using a MALDI-TOF mass spectrometer (AB SCIEX TOF/TOF ${ }^{\mathrm{TM}} 5800$ ). Spectra were analysed by TOF/TOF ${ }^{\mathrm{TM}}$ Data Series Explorer ${ }^{\mathrm{TM}}$ software. A mass range of $100-900 \mathrm{~m} / \mathrm{z}$ was allocated to analysis $\mathrm{m} / \mathrm{z}$ peaks within total lipid content. Quantification of peak intensity (lipid area percent of peak) and normalisation was performed using the following calculation: 


$$
=\frac{\text { Lipid }(x) \text { area }}{\text { Sum of all lipid area in sample }} \times 100
$$

Data were expressed by the mean average of peaks in triplicate readings and associated standard error.

\subsubsection{FACS sorting}

Cells: CLU/GFP positive cells were sorted on a BD Influx FACS sorter, with 488 Lazer (525/35 filter) to excite GFP and 355 to excite DAPI (450/50 filter). Data analysis carried out on FlowJo V_10 program.

\subsubsection{Autophagy Assays}

Cells.: Autophagy was investigated in cells using Western Blot analysis of LC3-II. Western blot conditions were carried out as previously described in section 2.2.3, with a few modifications. $20 \mu \mathrm{g}$ protein was run on a $15 \%$ Bis-Tris gel and transferred to a $0.2 \mu \mathrm{m}$ pore PVDF membrane with a transfer time of $2.15 \mathrm{~h}$ at $40 \mathrm{~V}$ at room temperature. The primary anti-LC3 antibody dilution (1:1000) (Rabbit polyclonal anti-LC3, Novus, cat \# NB100-2220) was incubated at $4^{\circ} \mathrm{C}$ overnight for 12-16 h. Secondary anti-rabbit antibody was used at a 1:2000 dilution for $1 \mathrm{~h}$ at $4^{\circ} \mathrm{C}$ prior to visualisation.

Autophagy was also investigated in cells using Lysotracker Red, a stain that is specific for acidified organelles. SH-SY5Y cells were grown in $\mu-2$ well confocal dishes to $70-80 \%$ confluency, and specific wells were transfected the following day. To select wells, Bafilomycin (10 nM) was then added and incubated for 16 h. $1 \mathrm{~mL}$ of 100nM Lysotracker Red (1:10,000 dilution of main stock) in RPMI media was then added and incubated for $2 \mathrm{~h}$ at 37 degrees with $5 \% \mathrm{CO}_{2}$. Cells were then washed with $3 \mathrm{X} \mathrm{RPMI}$ and cells were live-imaged in the final wash solution. Cells were imaged on the Olympus FV-1000 confocal microscope. Parameters used for assay is as follows: Transfected CLU/GFP cells were viewed at 488 lazer, HV at 499, 5\% laser intensity. Lysotracker Red was viewed at 590 laser, HV at 480, 13\% laser intensity. Scan speed for both is set at 4 microseconds per pixel using the $60 x$ objective. 


\subsection{RESULTS}

\subsubsection{Generation of UASCLU Drosophila/hCLU DNA}

\subsubsection{Cloning}

Despite the evolutionary conservation of CLU among higher mammals, CLU is not conserved in D. melanogaster. This is experimentally advantageous as we aim to express human CLU in D. melanogaster, effects of any endogenous CLU will not have to be accounted. We chose to express CLU in an established fly model of Alzheimer's disease (AD), the APP/BACE fly that has human APP and BACE overexpressed. To express human CLU in the APP/BACE fly, CLU DNA needs to be generated in a form where it can be transcribed and translated within the fruit fly genome and be specifically expressed within the fly brain. In order to achieve the desired expression, there are two constructs that are required to generate transgenic clusterin flies that overexpress CLU. The first construct is the corrected (wild type) version of CLU inserted into pDNR-Dual; this is necessary because the commercial source of CLU contains an unwanted mutation. The corrected version of clusterin was then excised and ligated into a pUAST-attB vector, in which the attB site undergoes homologous recombination via PhilC31 integrase with an attP, which is located in the genome of the fly that will be microinjected with the CLU construct. A GAL4/UAS system is then implemented in order for clusterin to be expressed in the brain region of the fly (section 1.9.4).

\subsubsection{Creation of pDNR-Dual + corrected version clusterin construct}

First, a commercial source of the open reading frame of human CLU in pDNR-Dual was obtained (DNASU Plasmid Repository, cat\# HsCD00000239) in the form of bacterial glycerol stocks in $\mathrm{T} 1 / \mathrm{T} 5$ phage-resistant $\mathrm{DH} 5 \alpha$ strain of $E$. coli. There is one nucleotide substitution $(\mathrm{t} 17 \mathrm{~g})$ in this construct that leads to an amino acid change (LR6) in the signal sequence of CLU. Although the signal sequence is still predicted to be recognised and cleaved, I sought to correct the mutation in order to be confident that I am studying the effects of wild type CLU and not the effects of a mutant variant of CLU. According to the vector map 
supplied by DNASU, the 1350 bp clusterin ORF can be released from pDNR-Dual with Sall and Xbal as neither enzyme cuts internally in clusterin or anywhere else in the plasmid. Since the mutation is very close to the $5^{\prime}$ end, correction of the mutation can be easily achieved by incorporating the correct nucleotide and the Sall site into a $5^{\prime}$ primer and used in combination with a $3^{\prime}$ primer with a Smal site and the Expand High Fidelity PCR System to amplify a fragment of wild type CLU (Table 2). This fragment was then ligated into the pDNR-Dual/CLU plasmid, thus replacing the mutant CLU variant with the wild type CLU.

To generate the CLU PCR product (Figure 2.2B), I initially used the cycling parameters of $94^{\circ} \mathrm{C}$ for $2 \min (1 \mathrm{cycle}) ; 94^{\circ} \mathrm{C}$ for $15 \mathrm{sec}, 66^{\circ} \mathrm{C}$ for $30 \mathrm{sec}$ and $72^{\circ} \mathrm{C}$ for $45 \mathrm{sec}$ ( $3 \mathrm{cycles}) ; 94^{\circ} \mathrm{C}$ for $15 \mathrm{sec}, 72^{\circ} \mathrm{C}$ for $30 \mathrm{sec}(\mathrm{x} 2)(22 \mathrm{cycles})$; and $72^{\circ} \mathrm{C}$ for $7 \mathrm{sec}(1 \mathrm{cycle})$. However, this standard protocol did not result in a product, so slight adjustments were made to the cycles through increasing the cycle number and decreasing the annealing temperature. These PCR conditions also did not generate an amplicon.

To confirm that the plasmid was definitely pDNR-Dual-CLU, I prepared a new plasmid prep and digested $1 \mu \mathrm{g}$ with Smal and Xbal, for which the correct bands (5511 bp and $736 \mathrm{bp}$ ) were obtained (Figure 2.2A, lane 2). However Sall (expected bands $4882 \mathrm{bp}$ and $1365 \mathrm{bp}$ ) resulted in a partial digest (Figure 2.2, lane 3), which suggested that the Sall stock being used was not active. I thus ordered a new tube of Sall.

The pDNR-Dual-CLU DNA in the miniprep that was initially used as a template for PCR was present in low levels (Figure 2.2A, lane 5). I thus used quantified this plasmid concentration to be $8.7 \mathrm{ng} / \mu \mathrm{L}$. Regardless of reduced levels, the quantity should be sufficient enough to enable PCR replication of the CLU gene. A new miniprep was prepared at a concentration of $108.3 \mathrm{ng} / \mu \mathrm{L}$ and the PCR protocol was changed (see section 2.2.2) so that the annealing temperature was reduced to $55^{\circ} \mathrm{C}$ and $63^{\circ} \mathrm{C}$ (instead of $66^{\circ} \mathrm{C}$ and $72^{\circ} \mathrm{C}$ for $30 \mathrm{sec}$ each) and cycles increased to 32 (instead of 22 cycles). The dilutions of the primers and plasmid were also increased to increase yield of PCR product. The newly developed PCR cycling protocol resulted in amplification of a $625 \mathrm{bp}$ product of the correct size (Figure 2.2B, lane 2). 
Once the 625 bp CLU fragment had been amplified through PCR, the PCR product was digested with Sall and Smal, while the pDNR-Dual-CLU vector was also cut with Sall and Smal. Each digestion was then treated with CIP. These digests were run on a gel (Figure $2.2 \mathrm{C}$ ) and the PCR product and the vector band were gel purified (Figure 2.2D). Then $1 \mu \mathrm{L}$ vector and $5 \mu \mathrm{L}$ of PCR product were ligated using the Quick Ligation Kit and an aliquot of the ligation mix was transformed into competent E.coli cells. The transformation yielded five colonies that were resistant to ampicillin, and plasmids were extracted from these transformants using the PureLink Quick plasmid miniprep kit.

To confirm the leucine mutation had been corrected and that only this version had been incorporated into the purified pDNR-Dual vector, I conducted a diagnostic restriction digest using the restriction enzymes BamHI and $\mathrm{Xbal}$. Since BamHI was included in the primer that amplified the corrected CLU amplicon, it will only be present in the plasmid with the corrected version of

A

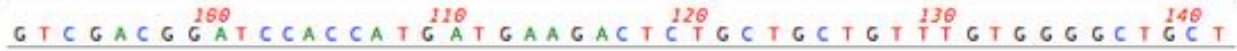

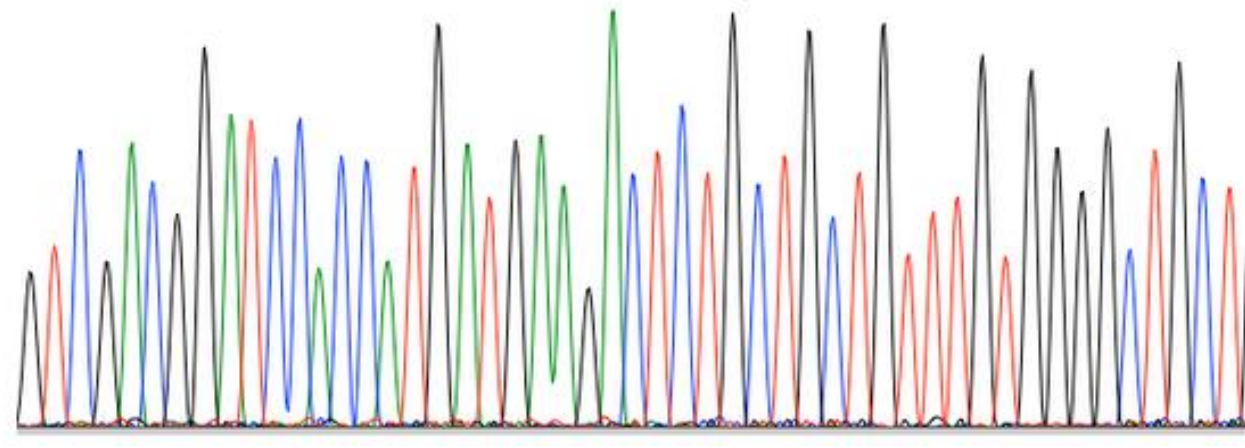

B

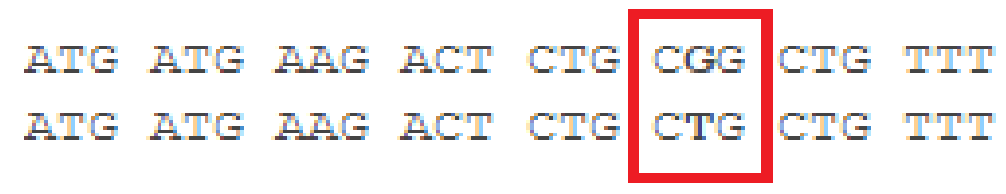

Figure 2.1: Sequence confirmation of corrected CLU fragment within pDNR-Dual + CLU (A) Chromatograph of clone \#1 with t17g mutation corrected. The correct nucleotide lines up at site amino acid site 123. (B) Sequence line up of incorrected (top line) and corrected (bottom line) sequences. CGG codes for arginine and CTG codes for leucine. The incorrect clone has a L6R amino acid substitution. An ATG codon is located nine amino acids before mutation. 
clusterin. Bands produced were $4919 \mathrm{bp}$ and $1328 \mathrm{bp}$ (Figure 2.4A), and these were the expected sizes. To confirm there were no other mutations incorporated within the CLU fragment during the PCR reaction, the plasmids were sequenced (Massey University) and the resulting sequence did not identify any additional mutations and confirmed the mutant variant was corrected in all five transformants (Figure 2.1). Concentrations of each plasmid were quantified using a nanodrop, and sample $1(156.8 \mathrm{ng} / \mu \mathrm{L})$ was used for further experiments (Appendix, Figure A1). 
A

4000bp

2000bp

$1650 \mathrm{bp}$

$1000 \mathrm{bp}$

$850 \mathrm{bp}$

$650 \mathrm{bp}$
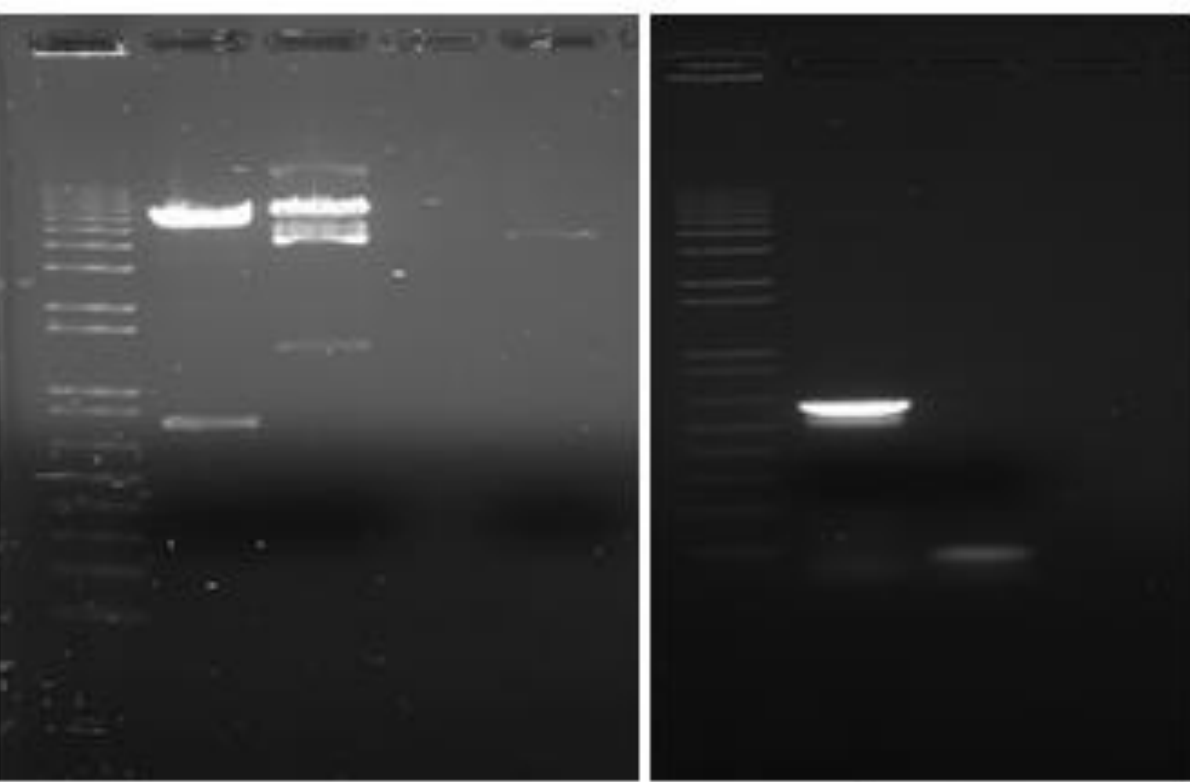

B
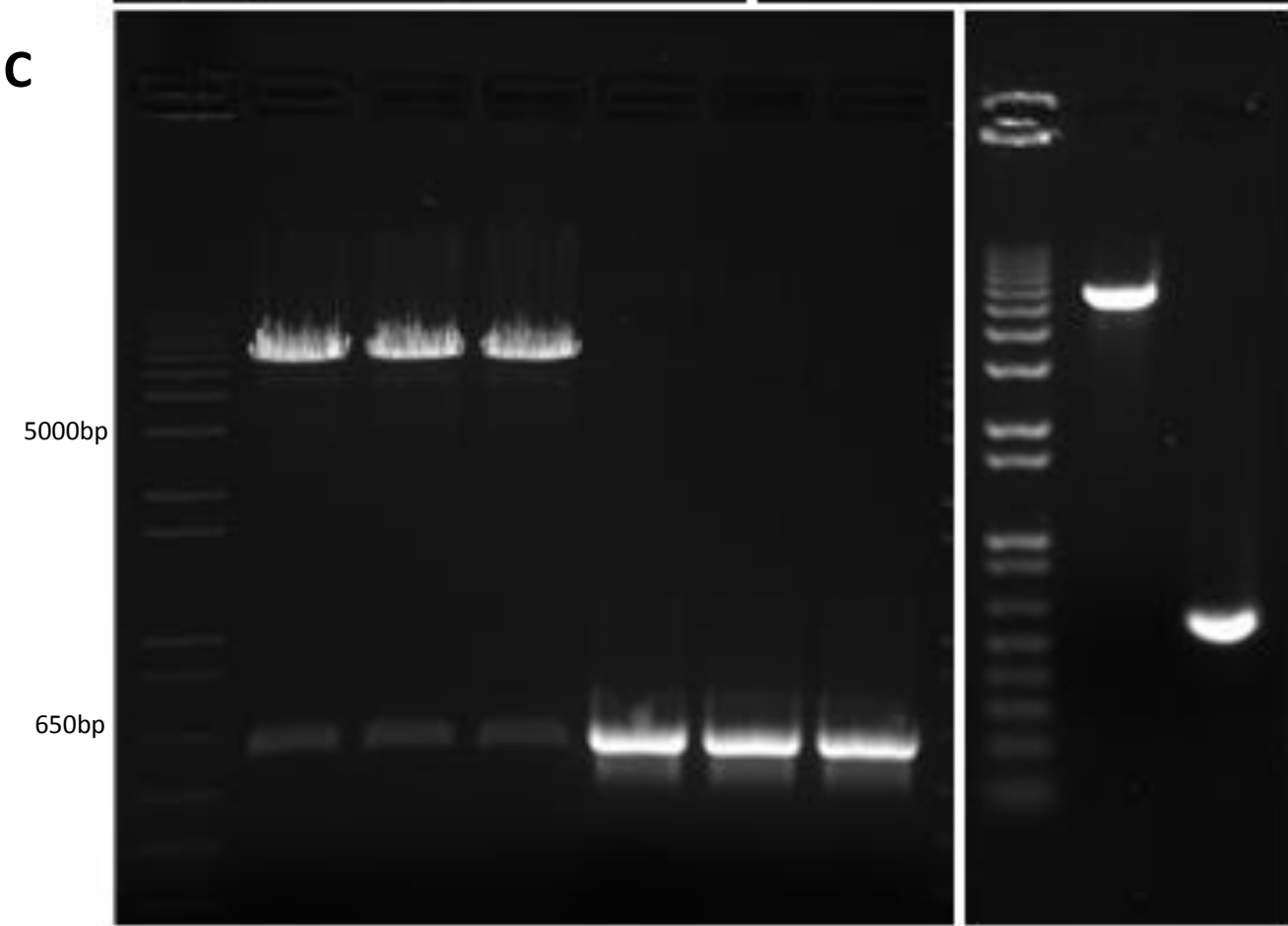

D

Figure 2.2: Correction of CLU mutation in pDNR-Dual + CLU vector

(A) Restriction Digest on pDNR- dual/CLU vector; lane 1: $8 \mu \mathrm{L}$ ladder, lane 2: $5 \mu \mathrm{L}$ Smal and Xbal digest, lane 3: $5 \mu \mathrm{L}$ Sall and Xbal digest, lane 4: blank, lane 5: $5 \mu \mathrm{L}$ previous CLU miniprep (B) PCR amplification of pDNR-Dual/CLU construct; lane 1: $8 \mu \mathrm{L}$ ladder, lane 2: $5 \mu \mathrm{L} \mathrm{CLU}$ template (band size $625 \mathrm{bp}$ - includes linkers on primers), lane 3: $5 \mu \mathrm{L}$ non-template (control) (C) Restriction digests of pDNR-Dual and CLU (Smal/Sall). Lanes 1-3: $5 \mu \mathrm{L}$ vector (5622bp), lanes 4-6: $5 \mu \mathrm{L}$ insert (625bp) (D) Purification of PCR product(CLU) and vector (pDNR-Dual); lane 1: $8 \mu \mathrm{L}$ ladder, lane 2: $5 \mu \mathrm{L}$ vector (5622bp), lane 3: $5 \mu \mathrm{L}$ insert (625bp). 


\subsubsection{Creation of pUAST-attB-CLU construct}

Once CLU has been corrected of the mutation, CLU DNA needs to be inserted into a vector that is compatible to be integrated into the fly CNS. To meet these needs, the pUAST-attB vector (Appendix, Figure A2) was utilized as it is specifically suited to a $D$. melanogaster model, as the attB site recombines with attP (via. PhilC31 integrase) to enable CLU to be taken up in the central nervous system (CNS) of APP/BACE flies by using the GAL4/UAS system. Without this driver system, expression of CLU DNA can't be achieved. To liberate the whole CLU ORF to insert it into pUAST-attB, I first digested pUAST-attB with Xhol and $\mathrm{Xbal}$ and CIP-treated the digest. Then I conducted a restriction digest on the pDNR-Dual/CLU construct with Sall and Xbal (Figure 2.4B). This combination of enzymes was chosen because Xhol and Sall have compatible sticky ends. Using the PureLink Gel Extraction Kit, the vector and insert were purified. A diagnostic gel was run to confirm the presence of pUAST-attB and pDNR-Dual/CLU after the gel extraction (Figure 2.4C).

Once the vector and insert were ligated, transformed and grown on LB agar plates containing ampicillin, restriction digests with Bglll were performed on six individual colonies to confirm that pUAST-attB and CLU have correctly been ligated. The restriction digest of all five clones was correct, with the vector (pUAST-attB) located as the top band ( $8800 \mathrm{bp}$ ) and the insert (clusterin) being the bottom band (943 bp) (Figure 2.4D). Further restriction digests were performed with BamHI and Smal on clone \#1 to further confirm that the clone was correct. Both restriction enzymes seem to cut at the correct position (Figure 2.4E), indicating that the pUAST-attB and CLU has successfully been incorporated into the pUAST-attB vector (Appendix, Figure A3).

\subsubsection{Generation of the UAS-CLU Drosophila melanogaster strain}

With the construction of the pUAST-attB-CLU construct, CLU is now in a form to be inserted into $D$. melanogaster by microinjection of DNA into fly embryos that will overexpress CLU as developing larvae mature into adult flies. The pUAST-attB-CLU DNA was injected into the $D$. melanogaster genotype y $\mathrm{w}, \mathrm{P}$ 
\{hs-flp\}; P $\{3 \times$ P3-RFP=attP-86F $\} ; P\{3 \times P 3-R F P=$ phic-31 $\{3 \times P 3-G F P=$ vas-phic31 $\}$ 102F' 'to generate a line that contains the UAS-CLU construct. The particular genotype that was used for microinjection resulted in a fly that has white eyes and a yellow (y) body. The attP site was tagged with RFP (red fluorescent protein) under the control of an eye specific promoter (3XP3) so it can be checked that this fly strain contains attP by checking that their eyes fluoresce red under the fluorescent microscope. PhilC31 integrase, which catalyses the homologous recombination between attP and attB, and is also responsible for integrating the CLU DNA into the fly, was inserted with the 3XP3-GFP. This was done so the presence of PhilC31 can be detected by checking that the eyes of the fly strain fluoresce green under the fluorescent microscope. Once the fly strain (UAS-CLU) was developed, the fly was crossed to a fly strain that contains the elav-GAL4 promotor (genotype'P\{w[+mW.hs]=GawB\}elav[C155]') in order for CLU to be specifically expressed within the CNS of the fly.

Microinjection using the pUAST-attB-CLU construct (the injection target in the fly being the poleplasm where germ cells form) had been carried out across two consecutive days and the proportion of surviving pupae was examined. Overall there was a survival rate of $25 \%$. Viable larvae were then grown at the appropriate temperatures with larvae initially grown at $25^{\circ} \mathrm{C}$, however that was subsequently dropped to $22^{\circ} \mathrm{C}$ due to high mortality rate of flies at $25^{\circ} \mathrm{C}$. During dechorionation and dehydration, these processes were dependent on each other in that the length of dehydration time was dependent on the amount of dechorionation time. These processes are known to affect the success rates of microinjection and in my case the incorporation of CLU into the wild type $D$. melanogaster. Eggs that are too dehydrated are unlikely to survive, and eggs that are too old are destroyed to lower the risk of contaminating the successful development of transgenic CLU flies. Overall, my microinjection experiment was successful in that I recovered 50 embryos that survived the microinjection process.

The developing larvae then took 10 days to mature into $\mathrm{G} 0$ adults. Males and females were then outcrossed to white-eyed wild-type flies (wcs10) of the appropriate sex. Transgenic progeny (G1) of this cross were selected by eye 
colour using the following criteria: transgenic flies were w- and had white eyes whereas transgenic flies had the $w+$ gene and had red eye colour restored. These flies were then outcrossed for four more generations in order to place the transgene in the same genetic background as the other lines that will be used in subsequent experiments.

\subsubsection{Generation of UAS-APP695-BACE-CLU Drosophila melanogaster strains}

In order to generate flies that overexpress UAS-APP695, UAS-BACE and UAS-CLU, four additional crosses were performed (Figure 2.3). Balancer genes tubby and curly ( $\mathrm{Tb}$ and $\mathrm{Cy}$ ) were used to track the specific genotypes in the crosses undertaken (as balancer genes are chromosomes that have undergone numerous rejoinings and breaks, these loci can no longer recombine to their original state $)^{102}$. The phenotypes of tubby and curly were fat pupae and curly

A finstcross

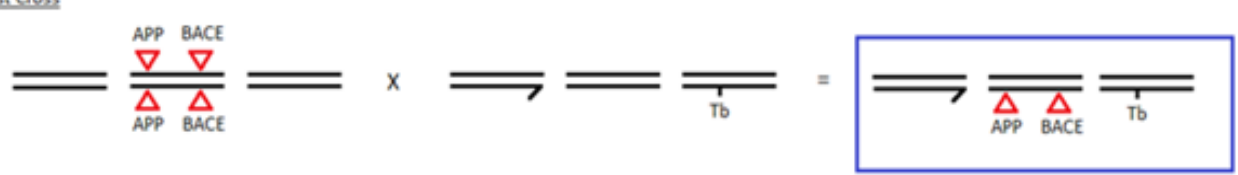

B Second Cross

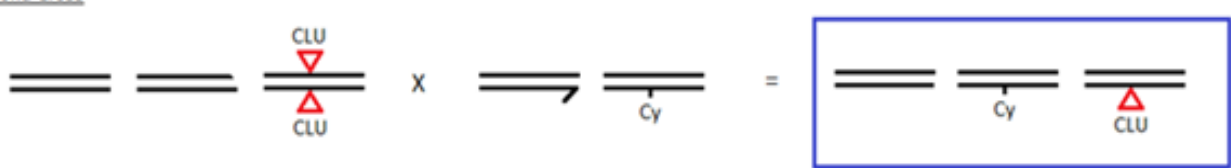

C Ihird Cross

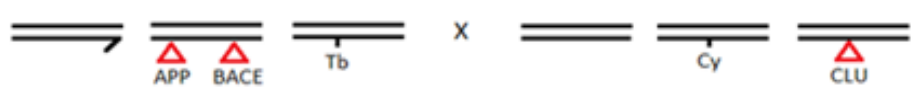

D Fourth Cross

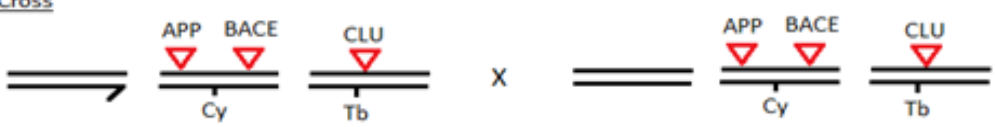

Figure 2.3: Generation of APP695/BACE/CLU D. melanogaster mutants (crosses)

(A) Female APP695/BACE drosophila crossed with male w(CS10) with Tb balancer gene, resulting in male APP695/BACE + Tb drosophila (B) female UAS-CLU fly crossed with male $w(C S 10)$ with Cy balancer gene, resulting in female UAS-CLU drosophila (C) male APP695/BACE + Tb drosophila crossed with female CLU + Cy drosophila. Selected for pupae and Cy wing adults (wild type eyes) (D) Male APP695/BACE/CLU + Tb and Cy drosophila crossed with female APP695/BACE/CLU + Tb and Cy drosophila. Need to select Non $\mathrm{Cy}$ and Non $\mathrm{Tb}$ - all should be homozygous. N.B. Full genotype of APP695/BACE flies is ' $w[1118] ; P\{w[+m C]=U A S-A P P 695-N-m y c\} T W 6, P\{w[+m C]=U A S-$ BACE1\}1 b' 
winged flies, respectively. In addition, balancer genes were specifically used to identify female progeny, as the male ' $Y$ ' chromosome does not undergo recombination ${ }^{102}$. When these crosses were carried out; since selection was based on eye colour, a small proportion of flies may not have CLU and only have the APP695/BACE genotype (since APP695/BACE have dark eyes), therefore several single pair matings were required. After the progeny were collected, PCR amplification of corrected CLU construct in pUAST-attB/CLU vector that were microinjected into flies(using primers from correction of pDNR-Dual + CLU) was conducted to confirm all three mutations were indeed present. 


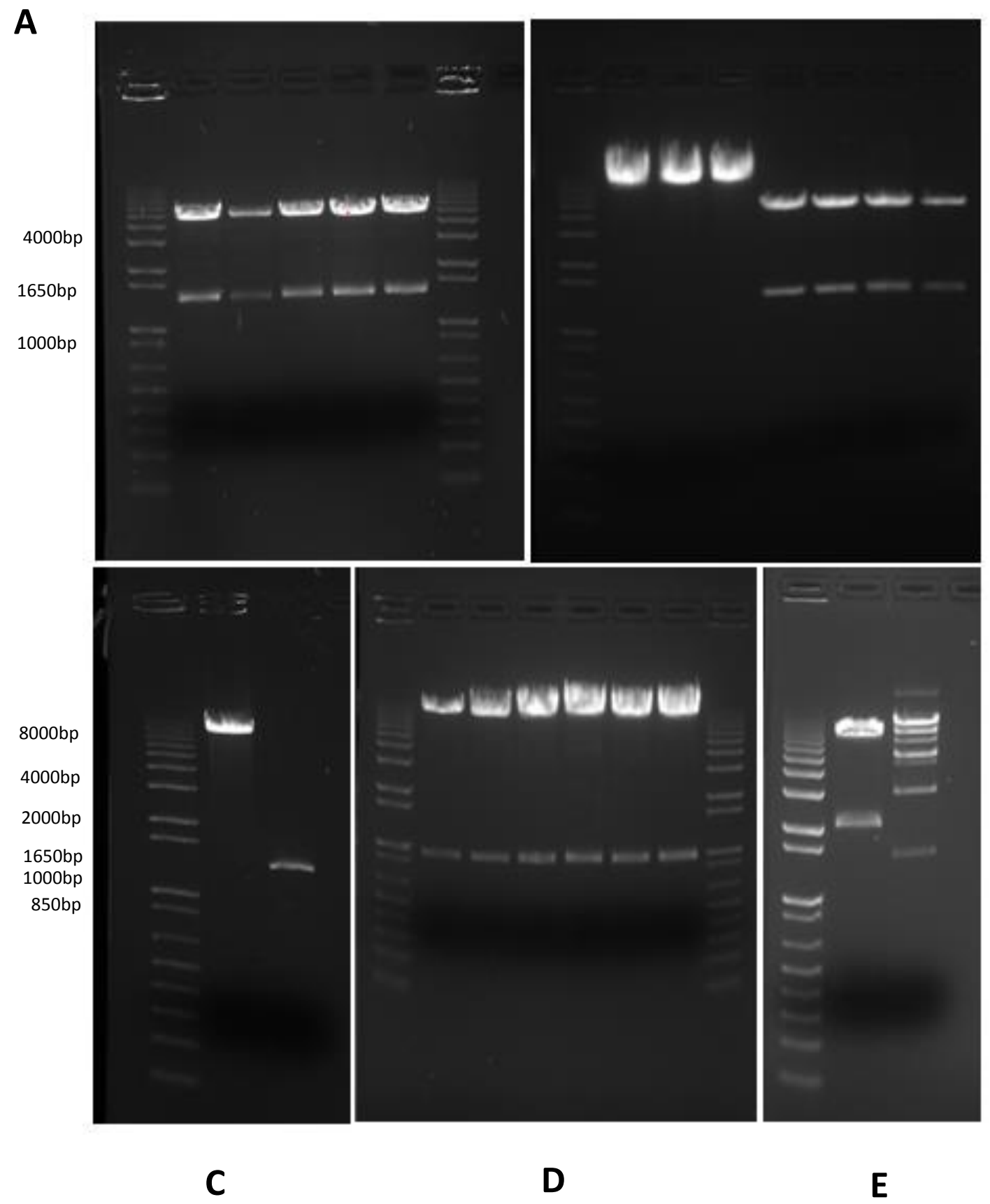

Figure 2.4: Creation of pUAST-attB + CLU vector

(A). Restriction Digests of corrected version of pDNR-Dual/CLU construct (BamHI/Xbal); Lane 1: $8 \mu \mathrm{L}$ ladder, lanes 2-6: $5 \mu \mathrm{L}$ pDNR-Dual CLU minipreps (1-5), lane 7: $8 \mu \mathrm{L}$ ladder (B) Restriction Digests of pUAST-attB (Xhol/Xbal) and pDNR-Dual + CLU (Sall/Xbal); lane 1: $8 \mu \mathrm{L}$ ladder, lane 2-4: $25 \mu \mathrm{L}$ vector (pUAST-attB) (bands $8477 \mathrm{bp}$ and 12bp), lanes 5-8: $25 \mu \mathrm{L}$ Insert (pDNR-Dual/CLU construct) (bands 4882bp and 1365bp) (C) Diagnostic PAGE; lane 1: $8 \mu \mathrm{L}$ ladder, lane 2: $5 \mu \mathrm{L}$ vector (pUAST-attB) (8477bp), lane 3: insert (pDNR-Dual/CLU) (1365bp) (D) Restriction digests using BgllI on pUASTattB hCLU plasmid; lane 1: $8 \mu \mathrm{L}$ ladder, lane 2-7: $5 \mu \mathrm{L}$ digests on pUAST-attB/CLU (1-6) (8899bp and 943bp), lane 8: $8 \mu \mathrm{L}$ ladder (E) Restriction Digest on pUAST-attB/CLU construct; lane 1: $8 \mu \mathrm{L}$ ladder, lane 2: $5 \mu \mathrm{L}$ Bgll digest (7358bp and 2484bp), lane 3: $5 \mu \mathrm{L}$ Smal digest (8299bp, 6796bp, 5253bp, 4589bp, 3046bp and 1543bp) 


\subsubsection{Generation of CLU in SH-SY5Y neuroblastoma cells}

\subsubsection{Cloning}

To express human CLU within SH-SY5Y and SH-SY5Y+SWAPP AD neuroblastoma cell lines, CLU was inserted into a vector specific for a mammalian system. For this to be achieved, two experimental reagents and one experimental optimization were required. First, the CLU fragment cloned into the fly vector pUAST-attB was excised and placed into the mammalian vector pcDNA3.1(+) between restriction sites Notl and Xbal (Appendix, Figure A4). Second, a reporter protein was added to the mammalian vector in order to detect positive transfection of CLU into SH-SY5Y cells. To do this, I cloned the GFP portion of RG203629 vector and ligated it into the pcDNA3.1(+)/CLU construct between restriction sites $\mathrm{Xbal}$ and Pmel. Third, optimisation of transfection was required in order to obtain the highest percentage of successful CLU overexpressing cells. This was determined through administering a range of concentrations of the transfection reagent Lipofectamine 2000 and CLU DNA, and determining the optimal ratios of Lipofectamine 2000 to DNA that achieved the highest yield of SH-SY5Y positive CLU expressing cells.

\subsubsection{Creation of pcDNA3.1(+) + clusterin construct}

To insert clusterin into a mammalian vector that is compatible with the SH-SY5Y cell line, I selected the vector pcDNA3.1(+) since this vector is used in common lab practice due to its ability to enable high expression levels within a variety of mammalian cell lines. The vector pcDNA3.1(+) and insert pUAST-attB + CLU was linearized with a restriction digest using Notl and Xbal and the vector CIP treated resulting in $5416 \mathrm{bp}$ and $1373 \mathrm{bp}$ fragments (Figure 2.5A, lane 45).Using Geneaid Gel/PCR DNA Fragments Extrtaction Kit, vector and insert bands were purified (Figure 2.4A, lane 2-3, Figure B). Using T4 DNA ligase, pcDNA3.1(+) and CLU were ligated with a $4 \mathrm{hr}$ incubation at $37^{\circ} \mathrm{C}$ and a diagnostic gel was run to confirm the presence of a successful ligated product. 


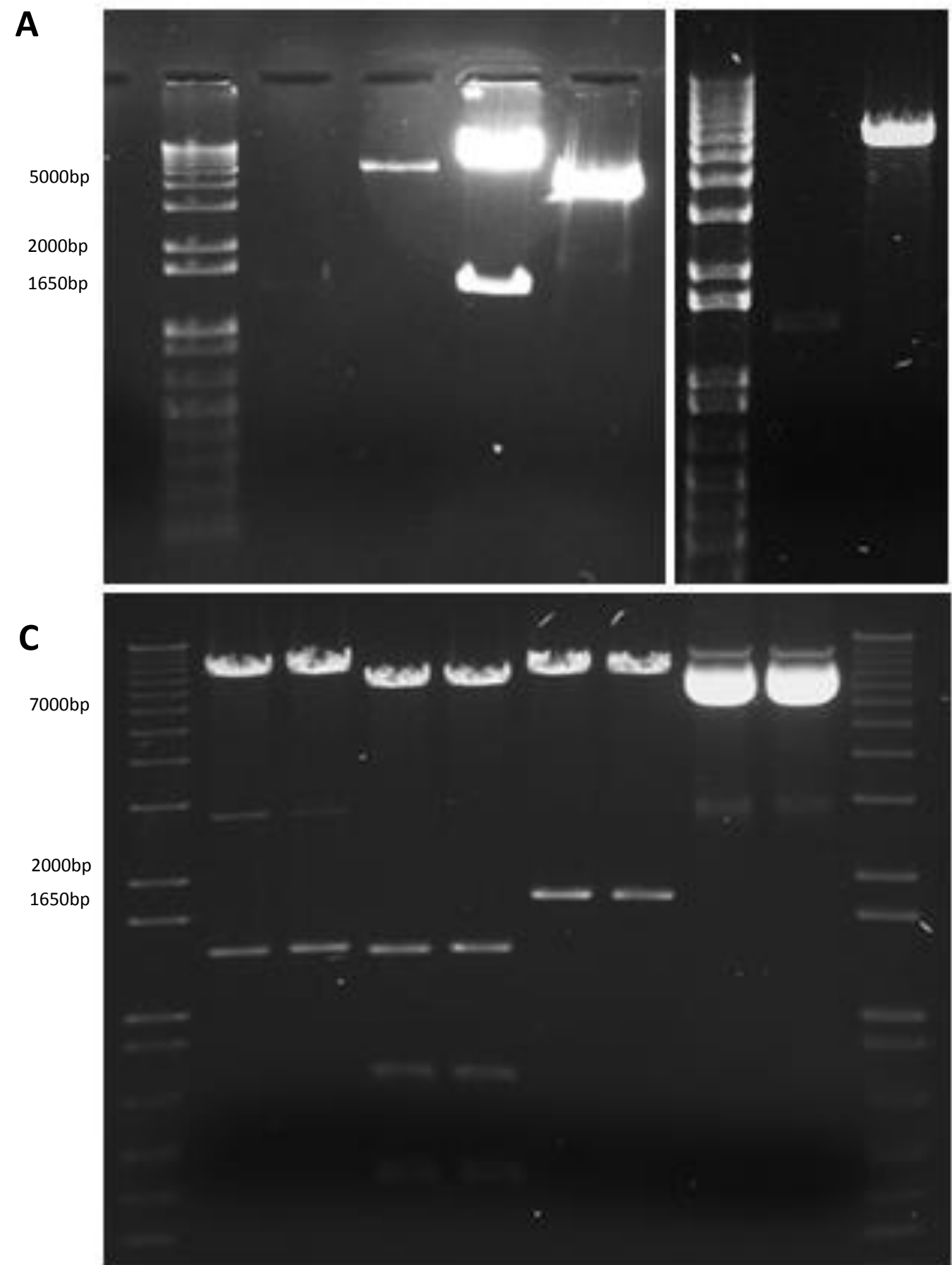

Figure 2.5: Creation of pcDNA3.1(+) + CLU construct

(A) Gel purification and restriction digests of pcDNA3.1(+) (vector) and insert (pUASTattB + CLU).Lane 1: ladder; lane2: gel purification of insert; lane 3: gel purification of vector; lane 4: restriction digest with Notl and Xbal on insert (pUAST-attB + $\mathrm{CLU})(1846 \mathrm{bp}$ and $1373 \mathrm{bp})$, lane 5: restriction digest with Notl and Xbal on vector (pcDNA3.1(+)) (7546bp and 12bp). (B) Confirmation of gel purified products lane 1: ladder; lane 2: Insert (CLU) (1373bp), lane 3: vector ( pcDNA3.1(+)) (5416bp). (C) Diagnostic restriction digests on ligated product. Lane 1: ladder, lane 2-3:Notl/Xbal digest (5416bp and 1373bp), lane 4-5BamHI/Xbal digest (5354bp and 1435bp); lane 67:HindIII/XBal digest (5336bp and 1453bp); lane 8-9:undigested ligated product (pcDNA3.1(+) + CLU) (6789bp); lane 10: ladder. 
However, since the presence of background bands in the gel suggested the ligation was partial; two different ligation methods were carried out at $26^{\circ} \mathrm{C}$ for 4 $\mathrm{hr}$ in addition to another ligation carried out at $4^{\circ} \mathrm{C}$ for $16 \mathrm{hr}$. The diagnostic gel to test out ligation products proved successful for both ligation conditions (Figure 2.5C, lane 7-8), demonstrating that the initial $4 \mathrm{hr}$ ligation at $37^{\circ} \mathrm{C}$ was inadequate for this particular ligation. The confirmed ligations were then transformed in competent E. coli cells, selected on ampicillin-containing media, miniprepped, and digested to confirm successful incorporation of CLU into pcDNA3.1(+) (Figure 2.5C, lanes 2-6).

\subsubsection{Integration of GFP in pcDNA3.1(+)/CLU construct}

Once the mammalian CLU plasmid was constructed, there was a requirement for a reporter gene to be present within the CLU mammalian vector. This is to determine successful incorporation of CLU in SH-SY5Y cells through the transfection technique with Lipofectamine 2000 as well as determining optimum transfection efficiencies within cells. The plasmid RG203629 was obtained (Appendix, Figure A5) and the GFP sequence from this particular vector was PCR amplified, resulting in a 705 bp fragment (Figure 2.6A). PCR products were gel purified, and digested along with pcDNA3.1 (+) + CLU (the mammalian construct created in section 2.4.2) with Xbal and Pmel restriction enzymes (Figure 2.6B). After incubation for $1 \mathrm{~h}$, the vector was CIP treated, and the required bands were gel purified and ligated. Ligation products were transformed in E. coli, selected on ampicillin-containing media, and plasmids were purified from transformants. Diagnostic restriction digests of plasmids were conducted to confirm successful ligation of GFP sequence and pcDNA3.1(+)/CLU (Figure 2.6C). 


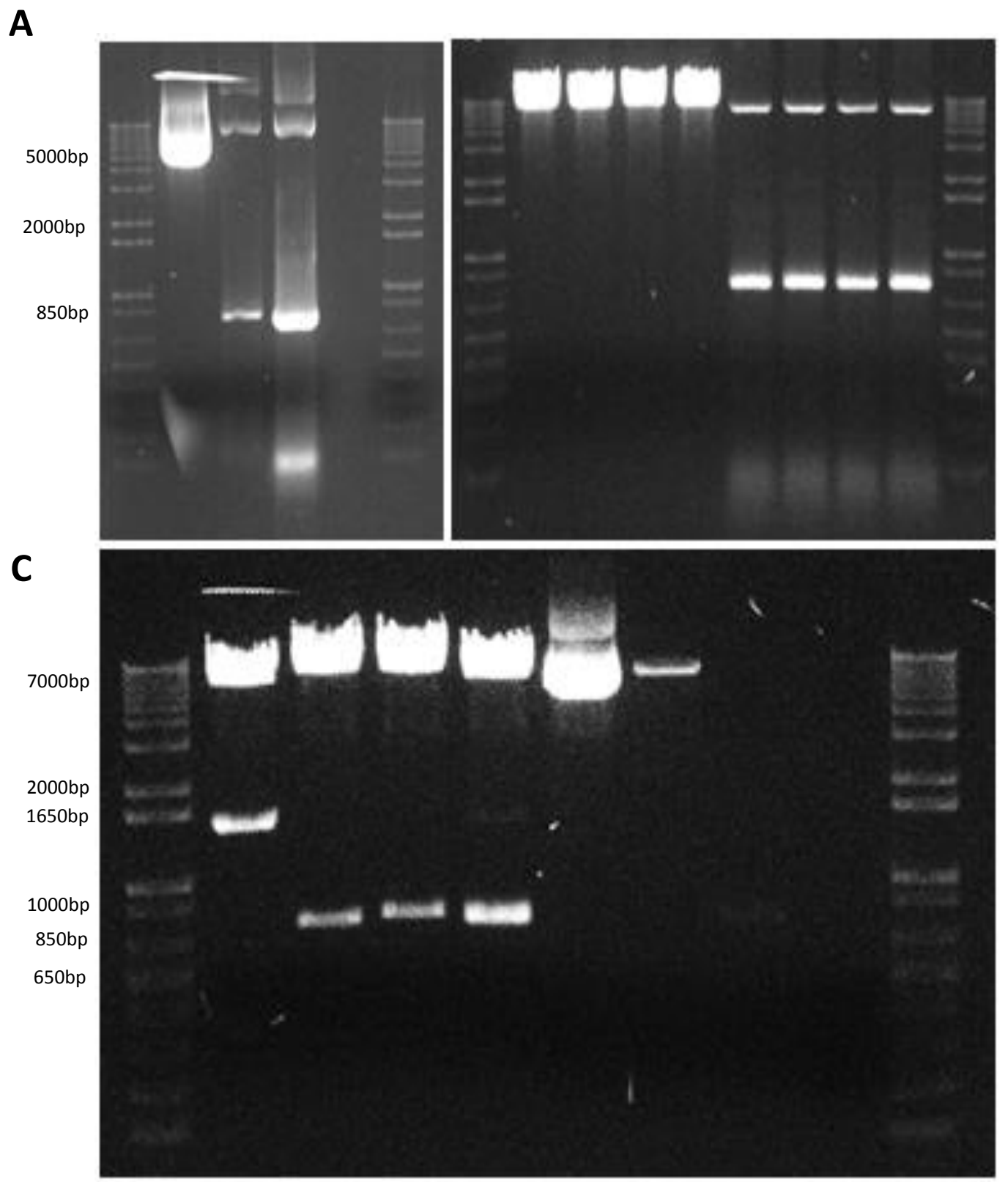

Figure 2.6: Creation of GFP + pcDNA3.1(+) CLU construct

(A) PCR gel of RG203629. Lane 1: Ladder, lane 2:undigested RG203629 (7900bp), lane 3:RG203629 GFP PCR product 1 (1ul); lane 4: RG203699 GFP PCR product 2 (5ul); lane 5: dye; lane 6: ladder ( 705bp) (B) Restriction digests of vector (pcDNA3.1(+) + CLU) and insert (RF=G203629) by Xbal and Pmel. Lane 1: Ladder, lane 2-5: restriction digests of vector (pcDNA3.1 $(+)+$ CLU), lane 6-9: restriction digest of insert (RG203629), lane 10: ladder. (C) Diagnostic restriction digests of ligated product (pcDNA3.1(+) + CLU + GFP). Lane 1:ladder; lane 2: Pmel/Notl digest ( 2078bp and 6789bp); lane 3: Notl/Xbal digest (7494bp and 1373bp); lane 4: Xbal/Pmel ( 705bp), lane 5: Xhol/Pmel ( 675bp); lane 6: complete plasmid uncut ( 8867bp), lane 7: gel purification of vector (8162bp), lane 8: gel purification of insert ( 705bp); lane 9: dye; lane 10: ladder. 


\subsubsection{Transfection optimisation of pcDNA3.1(+)/CLU/GFP in SH-SY5Y cell}

lines

The transfection reagent Lipofectamine 2000 was used to express the pcDNA3.1(+)/CLU/GFP construct into SH-SY5Y neuroblastoma cells. Lipofectamine 2000 transfects cells through a lipid-based mechanism in which the catatonic liposome formulation consists of positively charged lipids that have high binding affinity for negatively charged DNA. Therefore, Lipofectamine forms positively charged vesicles around the DNA of interest for cell uptake, as cell membranes also exhibit a negatively charged environment. This fusion across the cell membrane is mediated by the addition of the neutral lipid in Lipofectamine.

Given that previous literature indicates that SH-SY5Y cells are particularly hard to transfect with maximal transfection efficiencies at $30-40 \%$, it was critical that I optimise transfection conditions. Based from the manufacturer's instructions, SH-SY5Y cells were optimised using 3-6 $\mu$ L Lipofectamine 2000, 0.41.8 $\mu \mathrm{g}$ plasmid DNA (Figure 2.7-2.8). As optimal transfection efficiencies occur over a period of $24-48 \mathrm{~h}$, these parameters were adopted for wild type (Figures 2.9-2.12) and swAPP cells (Figures 2.13).

The best transfection efficiencies were achieved using $3 / 4,{ }_{8}$ and $1 / 2$ ratios with $24 \mathrm{~h}$ incubation. Further investigations were carried out by using different concentrations of 1:2 (DNA: Lipofectamine) transfection mix in order to optimise use of Lipofectamine product. (Ranges $3 / 4$ and ${ }^{9 / 8}$ ratios were investigated but proved to be inconsistent in transfection efficiencies produced). Further investigations of $1 / 2$ ratio proved successful with $1.6 \mu \mathrm{g}: 3.2 \mu \mathrm{L}$ and $2 \mu \mathrm{g}: 4 \mu \mathrm{L}$ diluted in $500 \mu \mathrm{L}: 100 \mu \mathrm{L}$ RPMI media (Figures 2.15-2.16). Transfection ratios incubated for $4 \mathrm{hr}$ and left to grow for $24 \mathrm{hr}$ at $2 \mu \mathrm{g}: 4 \mu \mathrm{L}$ (DNA/Lipofectamine) ratio proved best results for swAPP cells as cells can become $~ 90-100$ confluent at $48 \mathrm{hr}$ (Figure $2.15 \mathrm{C}$ ), whereas $48 \mathrm{hr}$ proved best results for WT SH-SY5Y cells (Figure 2.14D). 
A

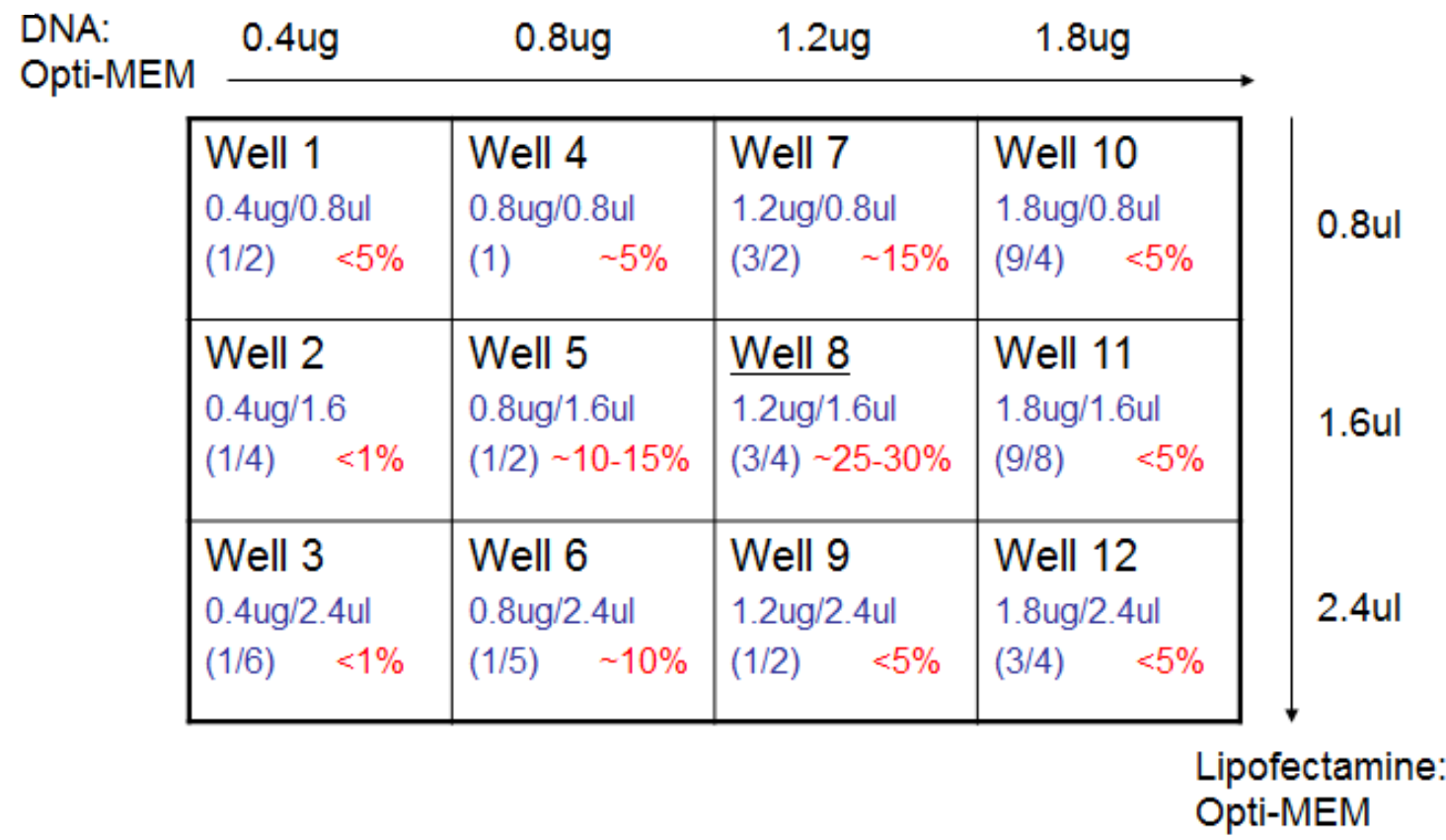

B

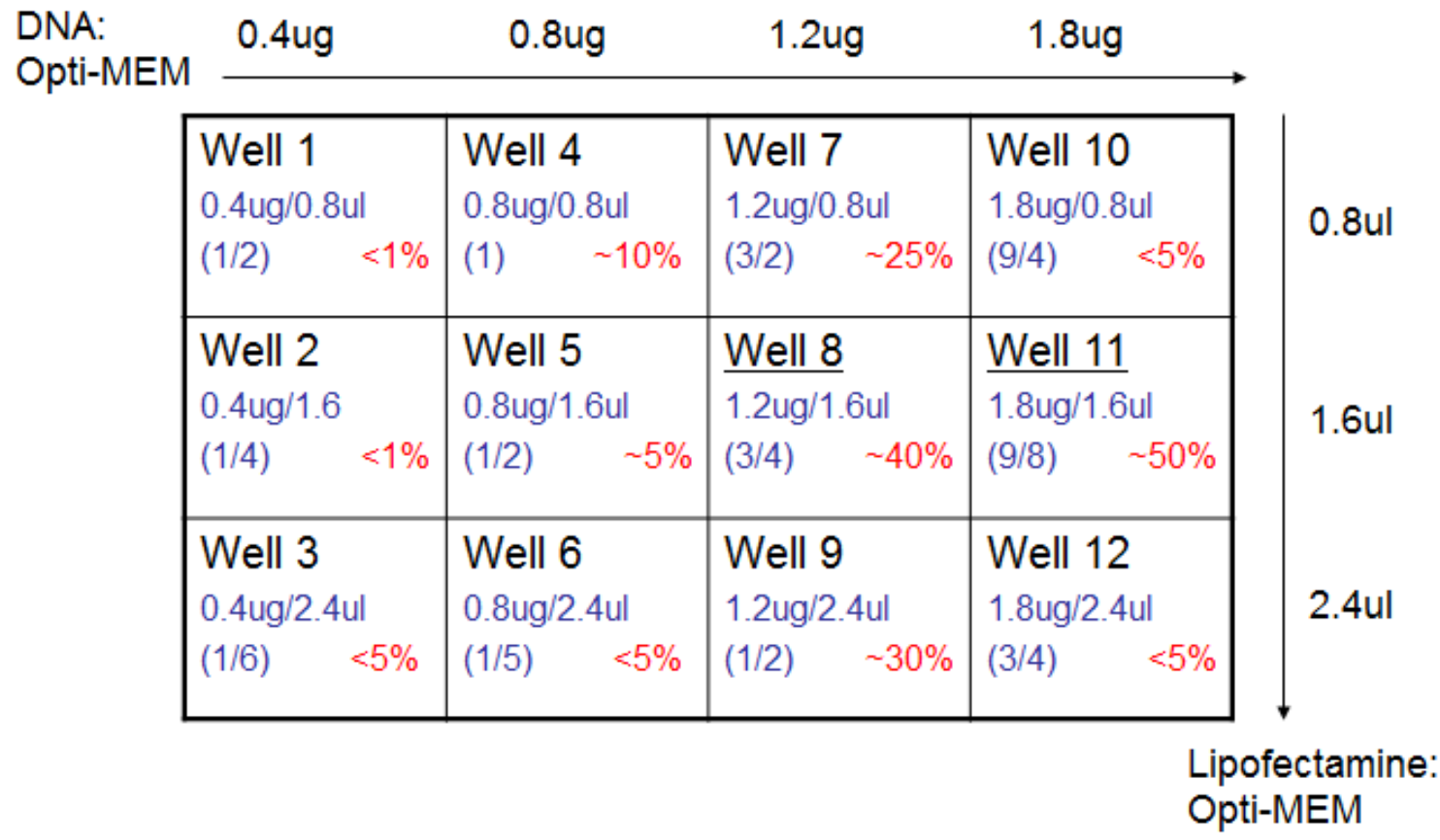

Figure 2.7: Experimental method for WT-SHSY5Y transfection optimisation

(A) Experimental procedure for examining WT SH-SY5Y transfection efficiency levels under different DNA:Lipofectamine 2000 conditions for 24hrs. (B) Experimental procedure for examining WT SH-SY5Y transfection efficiency levels under different DNA:Lipofectamine 2000 conditions under $48 \mathrm{hrs}$. 


\begin{tabular}{|c|c|c|c|c|c|}
\hline DNA: & 0.4 ug & 0.8 ug & $1.2 \mathrm{ug}$ & $1.8 \mathrm{ug}$ & \\
\hline & $\begin{array}{l}\text { Well } 1 \\
0.4 \mathrm{ug} / 0.8 \mathrm{ul} \\
(1 / 2)<1 \%\end{array}$ & $\begin{array}{l}\text { Well } 4 \\
0.8 \mathrm{ug} / 0.8 \mathrm{ul} \\
\text { (1) } \sim 5 \%\end{array}$ & $\begin{array}{l}\text { Well } 7 \\
1.2 \mathrm{ug} / 0.8 \mathrm{ul} \\
(3 / 2) \sim 10 \%\end{array}$ & $\begin{array}{l}\text { Well } 10 \\
1.8 \mathrm{ug} / 0.8 \mathrm{ul} \\
(9 / 4) \sim 5 \%\end{array}$ & $0.8 \mathrm{ul}$ \\
\hline & $\begin{array}{l}\text { Well } 2 \\
0.4 \mathrm{ug} / 1.6 \\
(1 / 4) \quad \sim 5 \%\end{array}$ & $\begin{array}{l}\text { Well } 5 \\
0.8 \mathrm{ug} / 1.6 \mathrm{ul} \\
(1 / 2) \sim 10 \%\end{array}$ & $\begin{array}{l}\text { Well } 8 \\
1.2 \mathrm{ug} / 1.6 \mathrm{ul} \\
(3 / 4) \sim 10 \%\end{array}$ & $\begin{array}{l}\text { Well } 11 \\
1.8 \mathrm{ug} / 1.6 \mathrm{ul} \\
(9 / 8) \sim 10 \%\end{array}$ & $1.6 \mathrm{ul}$ \\
\hline & $\begin{array}{l}\text { Well } 3 \\
0.4 \mathrm{ug} / 2.4 \mathrm{ul} \\
(1 / 6)<1 \%\end{array}$ & $\begin{array}{l}\text { Well } 6 \\
0.8 \mathrm{ug} / 2.4 \mathrm{ul} \\
(1 / 5) \sim 5 \%\end{array}$ & $\begin{array}{l}\text { Well } 9 \\
1.2 \mathrm{ug} / 2.4 \mathrm{ul} \\
(1 / 2) \sim 10 \%\end{array}$ & $\begin{array}{l}\text { Well } 12 \\
1.8 \mathrm{ug} / 2.4 \mathrm{ul} \\
(3 / 4) \sim 5 \%\end{array}$ & $2.4 \mathrm{ul}$ \\
\hline
\end{tabular}

Figure 2.8: Experimental method for SWAPP SH-SY5Y transfection optimisation Experimental procedure for examining WT SH-SY5Y transfection efficiency levels under different DNA:Lipofectamine 2000 conditions for $24 \mathrm{hrs}$. 
Weill 1

$0.4 u g / 0.8 u$

(1/2)

Well 2

$0.4 u g / 1.6 u$

(1/4)

Well 3

$0.4 u g / 2.4 u l$

$\langle 1 / 6\}$

Well 4

$0.8 \mathrm{Lg} / 0.8 \mathrm{u}$

(1)

Well 5

$0.8 u g / 1.6 u$

(1/2)

Well 6

$0.8 \mathrm{ug} / 2.4 \mathrm{ul}$

(1/3)

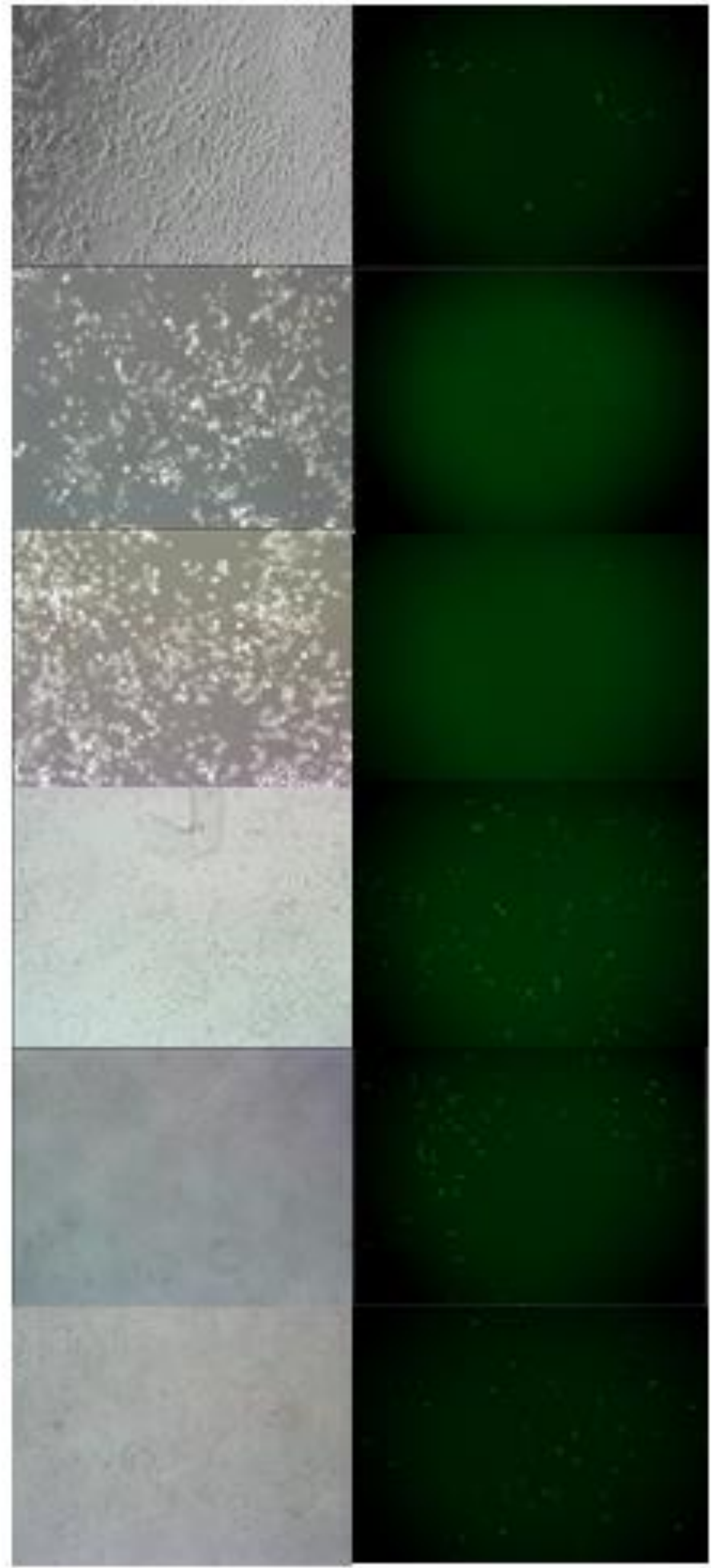

Figure 2.9: Transfection trial of WT-SHSY5Y cells incubated for $24 \mathrm{~h}$ (well 1-6)

Transfection media on WT SH-SY5Y cells incubated for a $4 \mathrm{~h}$ time period before replaced with RPMI $+10 \%$ FCS media. Ratios given beside each well is the amount of DNA to Lipofectamine 2000 amounts. 


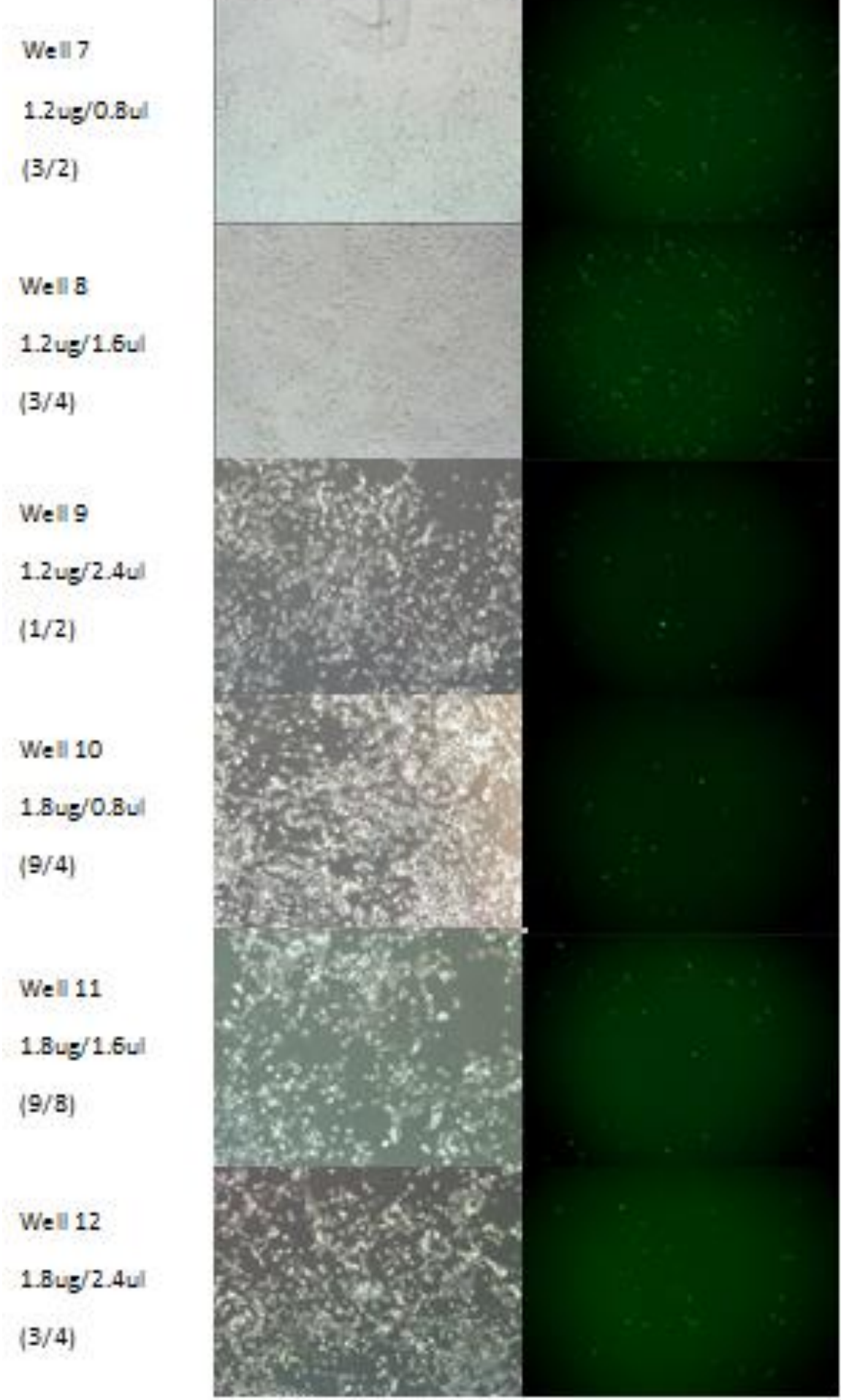

Figure 2.10: Transfection trials for WT SHSY5Y cells incubated for $24 \mathrm{~h}$ (well 7-12) Transfection media on WT SH-SY5Y cells incubated for a $4 \mathrm{~h}$ time period before replaced with RPMI $+10 \%$ FCS media. Ratios given beside each well is the amount of DNA to Lipofectamine 2000 amounts. 


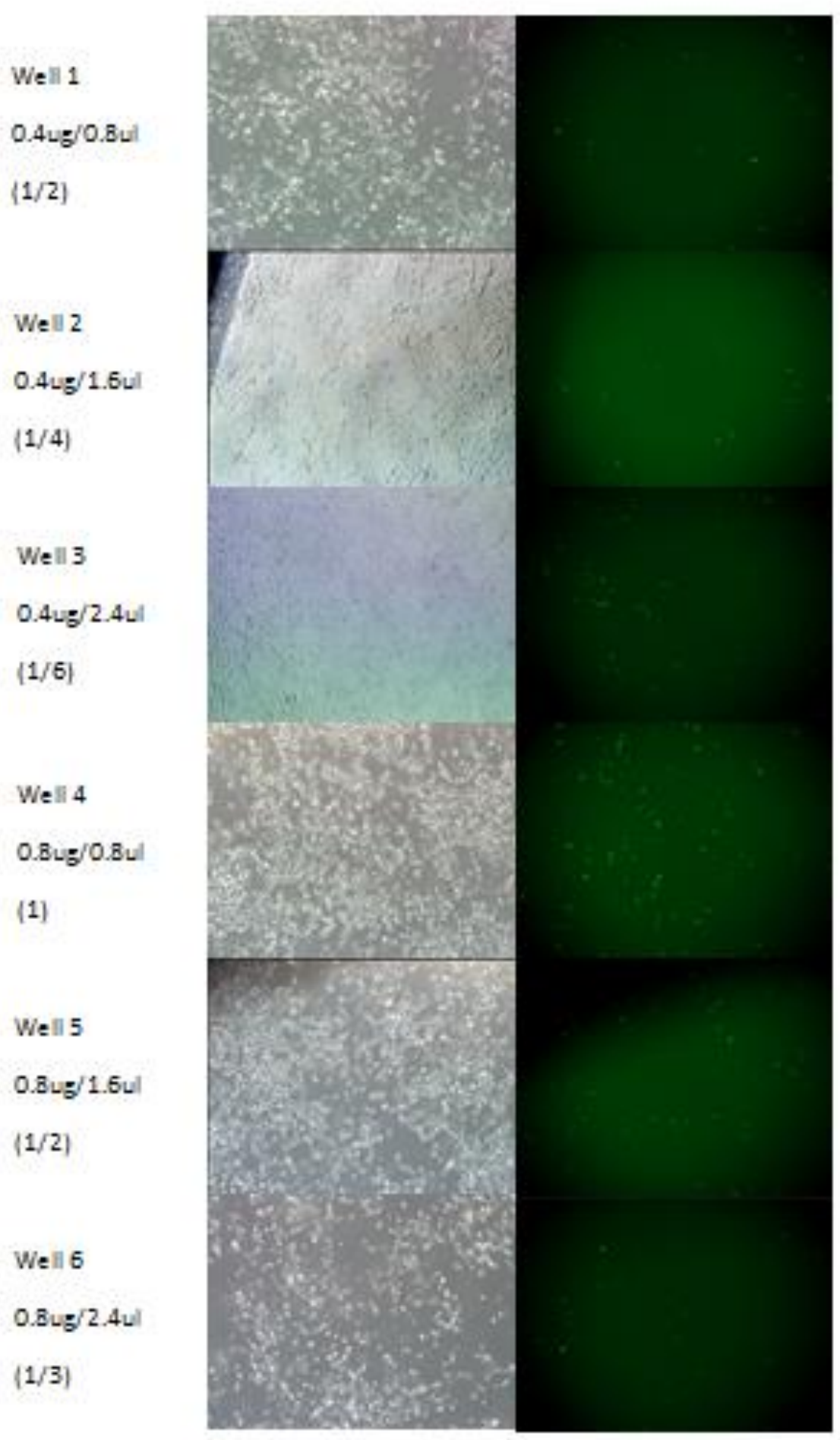

Figure 2.11: Transfection trials of WT SHSY5Y cells incubated for $48 \mathrm{~h}$ (well 1-6) Transfection media on WT SH-SY5Y cells incubated for a $4 \mathrm{~h}$ time period before replaced with RPMI $+10 \%$ FCS media. Ratios given beside each well is the amount of DNA to Lipofectamine 2000 amounts. 


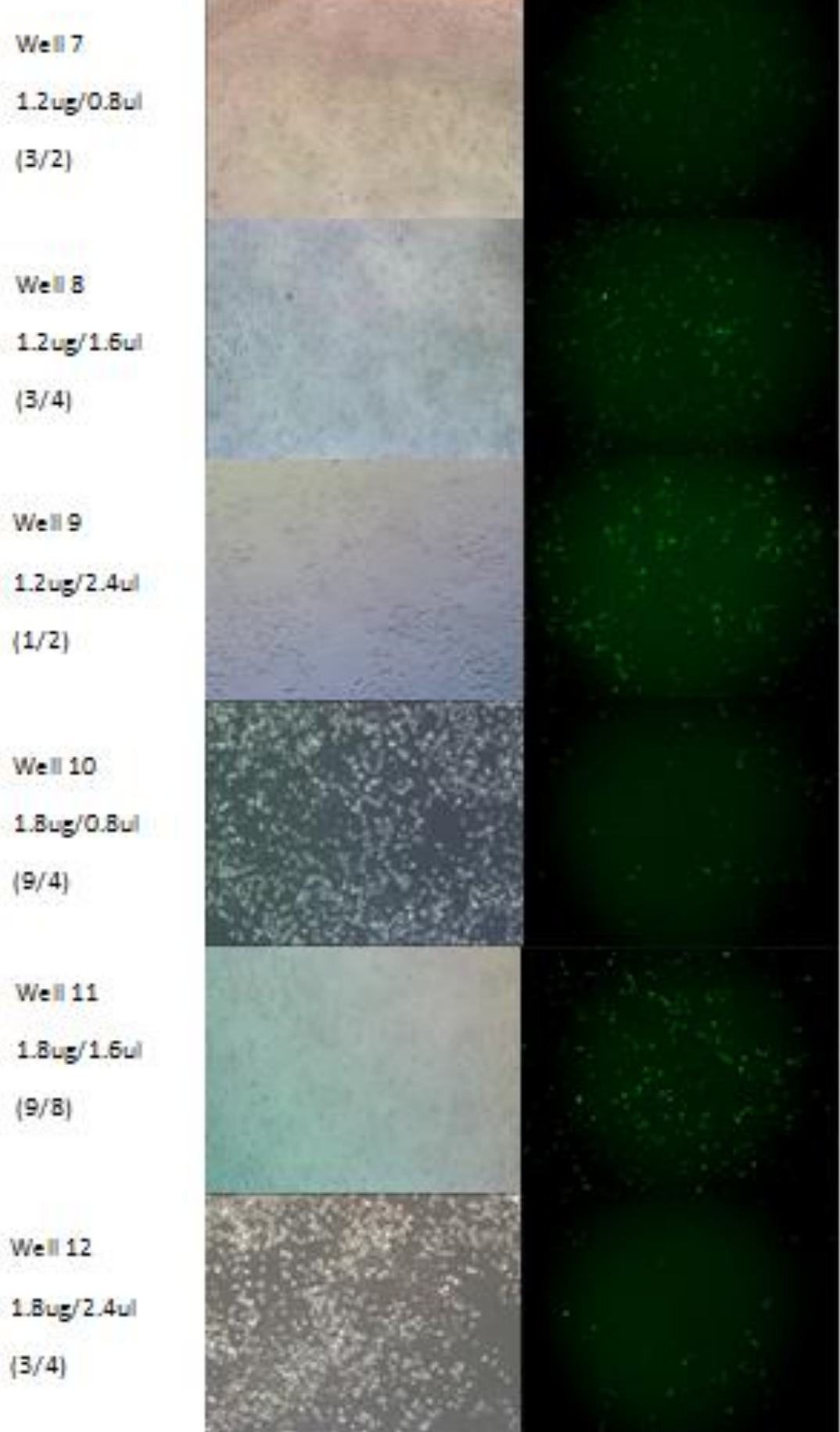

Figure 2.12: Transfection trials of WT SHSY5Y cells incubated for 48h (well 7-12) Transfection media on WT SH-SY5Y cells incubated for a $4 \mathrm{~h}$ time period before replaced with RPMI + 10\% FCS media. Ratios given beside each well is the amount of DNA to Lipofectamine 2000 amounts. 
Well 1

$0.4 \mathrm{~g} / 0.8 \mathrm{ul}$

(1/2)

Well 2

0.4 เg/1.6ul

Well 3

$0.4 u g / 2.4 u$

(1/6)

Well 4

$0.84 \mathrm{~g} / 0.8 u$

(1)

Well 5

$0.84 \mathrm{~g} / 1.6 u$

[1/2]

Well 6

$0.84 \mathrm{~g} / 2.4 u$

(1/3)

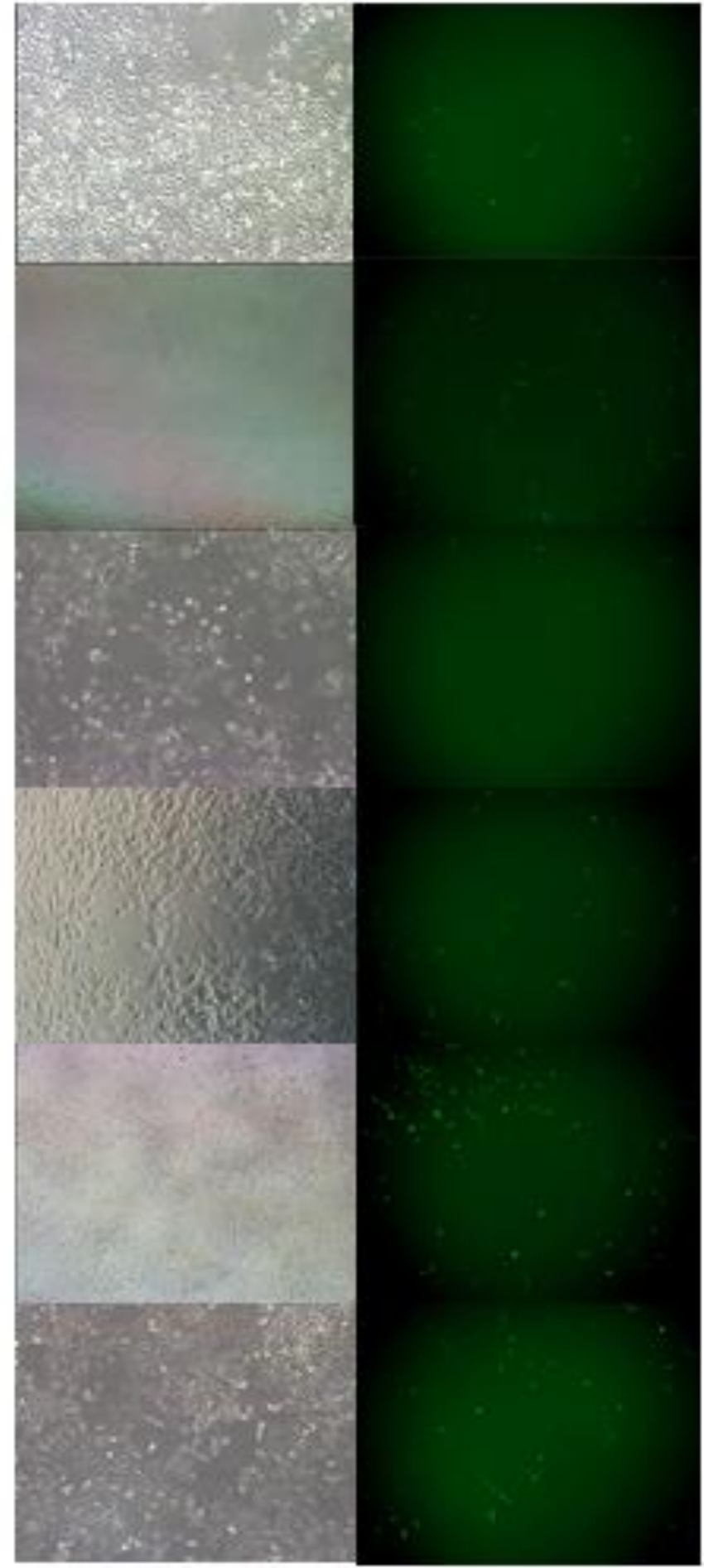

Figure 2.13: Transfection trials of swAPP SH-SY5Y cells incubated for $24 \mathrm{~h}$ (well 1-6) Transfection media on swAPP SH-SY5Y cells incubated for a $4 \mathrm{~h}$ time period before replaced with RPMI $+10 \%$ FCS media. Ratios given beside each well is the amount of DNA to Lipofectamine 2000 amounts. 


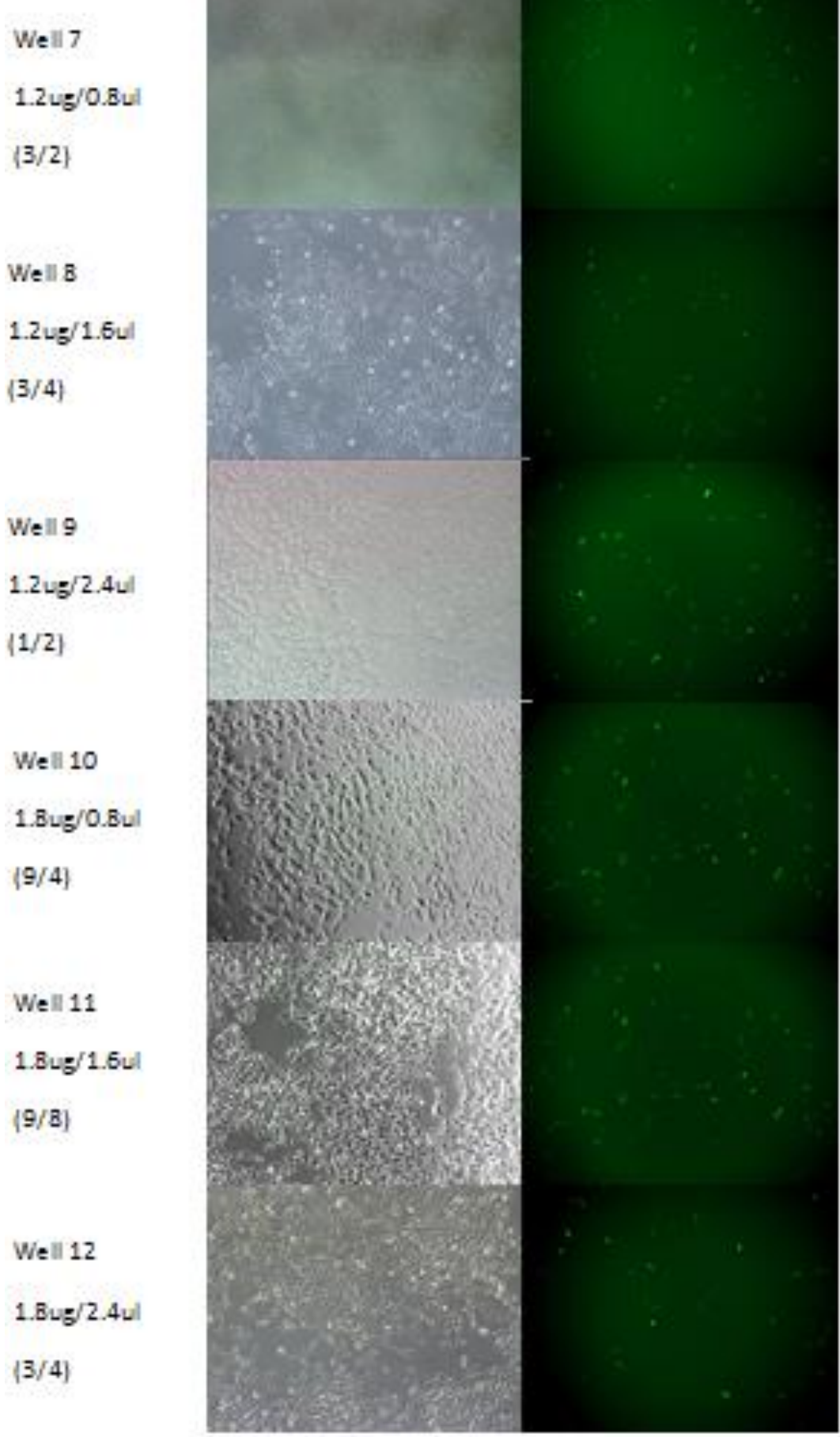

Figure 2.14: Transfection trials of swAPP SH-SY5Y cells incubated for $24 \mathrm{~h}$ (well 7-12) Transfection media on SWAPP SH-SY5Y cells incubated for a $4 \mathrm{~h}$ time period before replaced with RPMI $+10 \%$ FCS media. Ratios given beside each well is the amount of DNA to Lipofectamine 2000 amounts. 

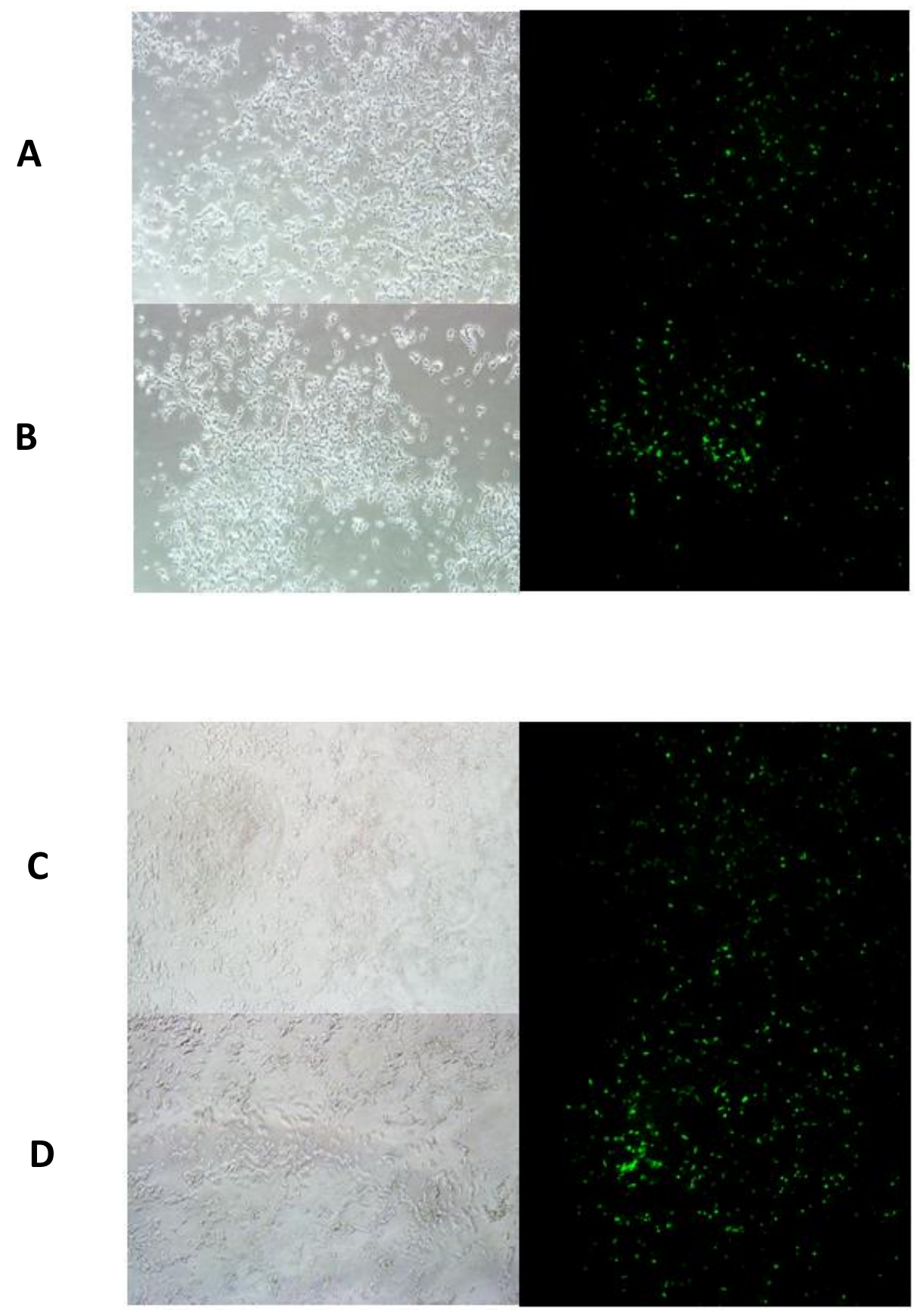

Figure 2.15: Transfection optimisation (1:2 DNA:Lipofectamine) in WT SH-SY5Y cells (A) Wild type cells treated with 1.6ug DNA: 3.2uL Lipofectamine over a $24 \mathrm{~h}$ period with a $\sim 10 \%$ efficiency (B) Wild type cells treated with 1.6ug DNA: 3.2uL Lipofectamine over a $48 \mathrm{~h}$ period with $\sim 15-20 \%$ efficiency (C) Wild type cells treated with 2ug DNA: 4uL Lipofectamine over a $24 \mathrm{hr}$ period with a $20 \%$ efficiency (D) Wild type cells treated with 2 ug DNA: 4 uL Lipofectamine over a $24 \mathrm{hr}$ period with a $40 \%$ efficiency. 

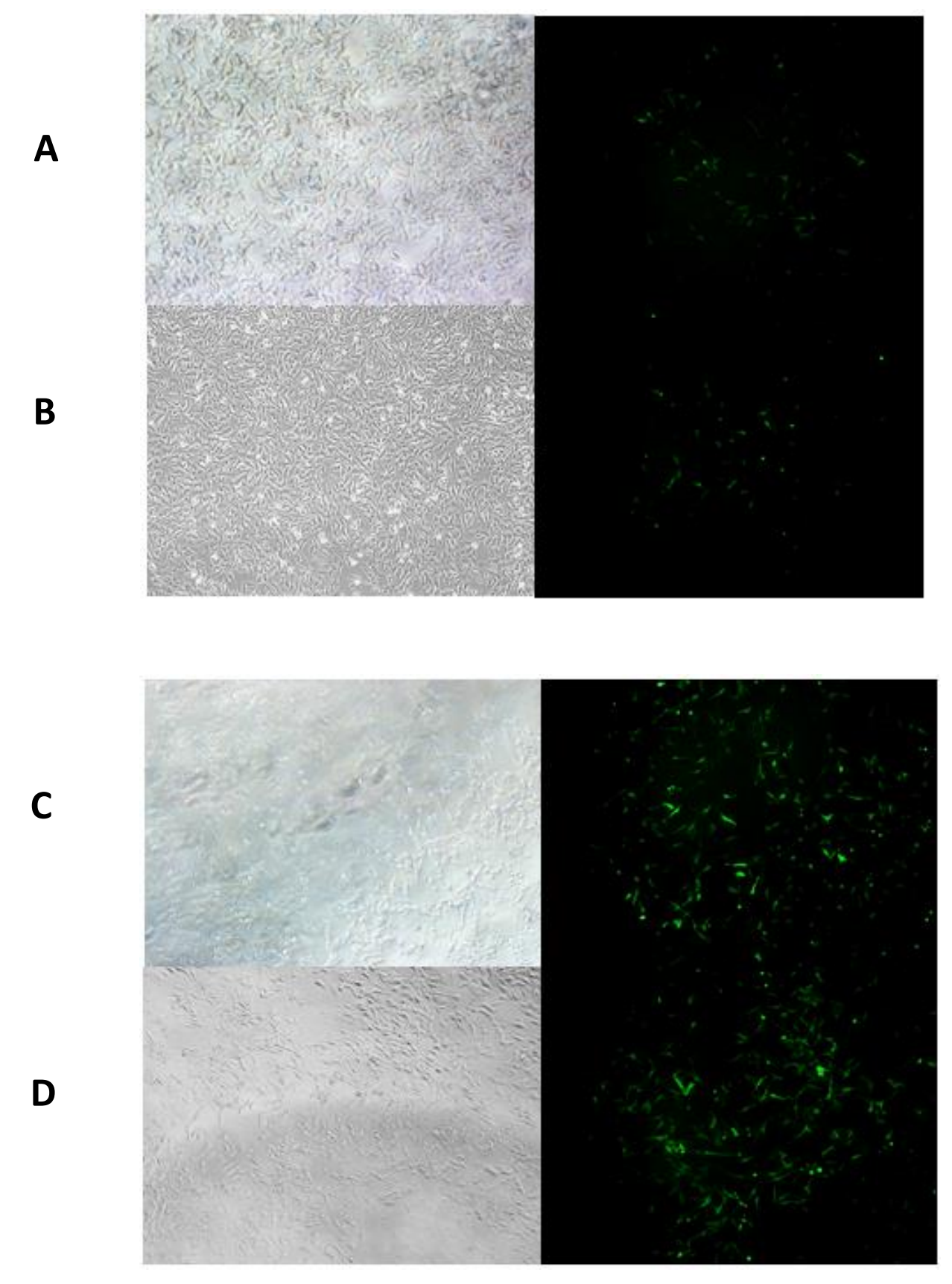

Figure 2.16: Transfection optimisation (1:2 DNA: Lipofectamine) in SWAPP SH-SY5Y cells Transfection optimisation (1:2 ratio;DNA:Lipofectamine) in SWAPP SH-SY5Y cells (A) swAPP cells treated with 1.6ug DNA: 3.2uL Lipofectamine over a $24 \mathrm{~h}$ period with a $<5 \%$ efficiency (B) swAPP cells treated with 1.6ug DNA: 3.2uL Lipofectamine over a $48 \mathrm{~h}$ period with $\sim 5-10 \%$ efficiency (C) swAPP cells treated with 2ug DNA: 4uL Lipofectamine over a $24 \mathrm{hr}$ period with a $20 \%$ efficiency (D) swAPP cells treated with $2 \mathrm{ug}$ DNA: $4 \mathrm{uL}$ Lipofectamine over a $24 \mathrm{hr}$ period with a $40 \%$ efficiency. 


\subsection{Clusterin Enhances Survival Rates in the APP/BACE Fruit fly Model of}

Alzheimer's disease

In $A D$, gross motor skills such as balancing and head movements as well as fine motor skills (e.g., coordination of body parts) are affected as the pathology of the disease increases over time. Upon diagnosis, the life expectancy after $A D$ is also decreased, with a shortened life expectancy roughly around 10 years. Due to these symptoms in humans, it was of particular interest to see if AD fruit flies exhibit similar patterns, as well as any other pathological symptoms during development with the APP/BACE expressed in flies. If APP/BACE fruit flies do exhibit this pattern, then deterioration of motor function would therefore be due to defects found within the central complex of the fruit fly brain; the region which provides motor output after the completion of sensory integration from other regions of the brain.

Commercially purchased APP/BACE flies were initially grown at $25^{\circ} \mathrm{C}$; however the survival rate of the flies was severely affected, as mortality rate was extremely high resulting in insignificant amounts of progeny generated for following experiments. In order to improve number of surviving progeny, APP/BACE flies were grown at $18^{\circ} \mathrm{C}$ and $22^{\circ} \mathrm{C}$ which greatly increased the number of offspring produced. This was most likely due to GAL4 functionality as it is more active at higher temperatures; therefore more APP695 and BACE were produced at $25^{\circ} \mathrm{C}$, which may have resulted in increased $A \beta 1-42$ toxicity. The resulting survival rates between male and female were the following: (WT) $=1: 02: 1$, $($ ELAV/CLU $)=1: 0.4: 1,($ APP/BACE $)=3: 74: 1,($ APP/BACE $/ C L U)=1: 52: 1$ 
2.5 Clusterin Ameliorates the Crumpled Wing Phenotype in the APP/BACE Fruit fly Model of Alzheimer's disease

Chakraborty and colleagues reported the crumpled wing phenotype portrayed in the APP/BACE AD fly (Figure 2.17B). The crumpled wing phenotype was also demonstrated within APP flies, however this phenotype was increased 10 -fold in APP/BACE flies ${ }^{98}$, which supports the notion that morphological defects are dependent on the processing of APP by BACE as well as the production of amyloid beta plaques. Indeed, the gamma secretase inhibitor L685/458 reduced the occurrence the crumpled wing phenotype in APP/BACE flies $^{98,105}$. Based on these reports, I predict that CLU overexpression should decrease the morphological defect of crumpled wings due to the protective properties of CLU.

Flies grown at $22^{\circ} \mathrm{C}$ that were collected a week apart were characterised for the crumpled wing phenotype. APP/BACE mutants did indeed contain crumpled wings with $96.9 \%$ of the male population and $31.7 \%$ female population exhibiting this pathological morphology (Figure 2.17C). However there seemed to be only a certain portion of APP/BACE progeny that had this phenotype. The other phenotype exhibited was no crumpled wings within the AD fly, however these flies should theoretically still have the APP695 genotype. This is interesting as pathological defects within the fly wing would be expected to occur in all APP/BACE genotypes. CLU expression within the APP/BACE flies decreased the crumpled wing phenotype in the male population by $31.4 \%$ (from $96.9 \%$ to $31.7 \%$ ) and by $17.7 \%$ (from $31.7 \%$ to $14 \%$ ) in the female population (Figure 2.17C). Therefore, the phenotype of crumpled wings seems to be more prominent within males compared to females. To support my observations, I monitored the phenotype in additional genotypes, and as expected, wild type and ELAV/CLU controls portrayed negative symptoms towards the crumpled wing phenotype (wild type males having $0.8 \%$ and females $0 \%$ crumpled wing phenotype and ELAV/CLU males having $0.6 \%$ and females $1 \%$ crumpled wing phenotype). 


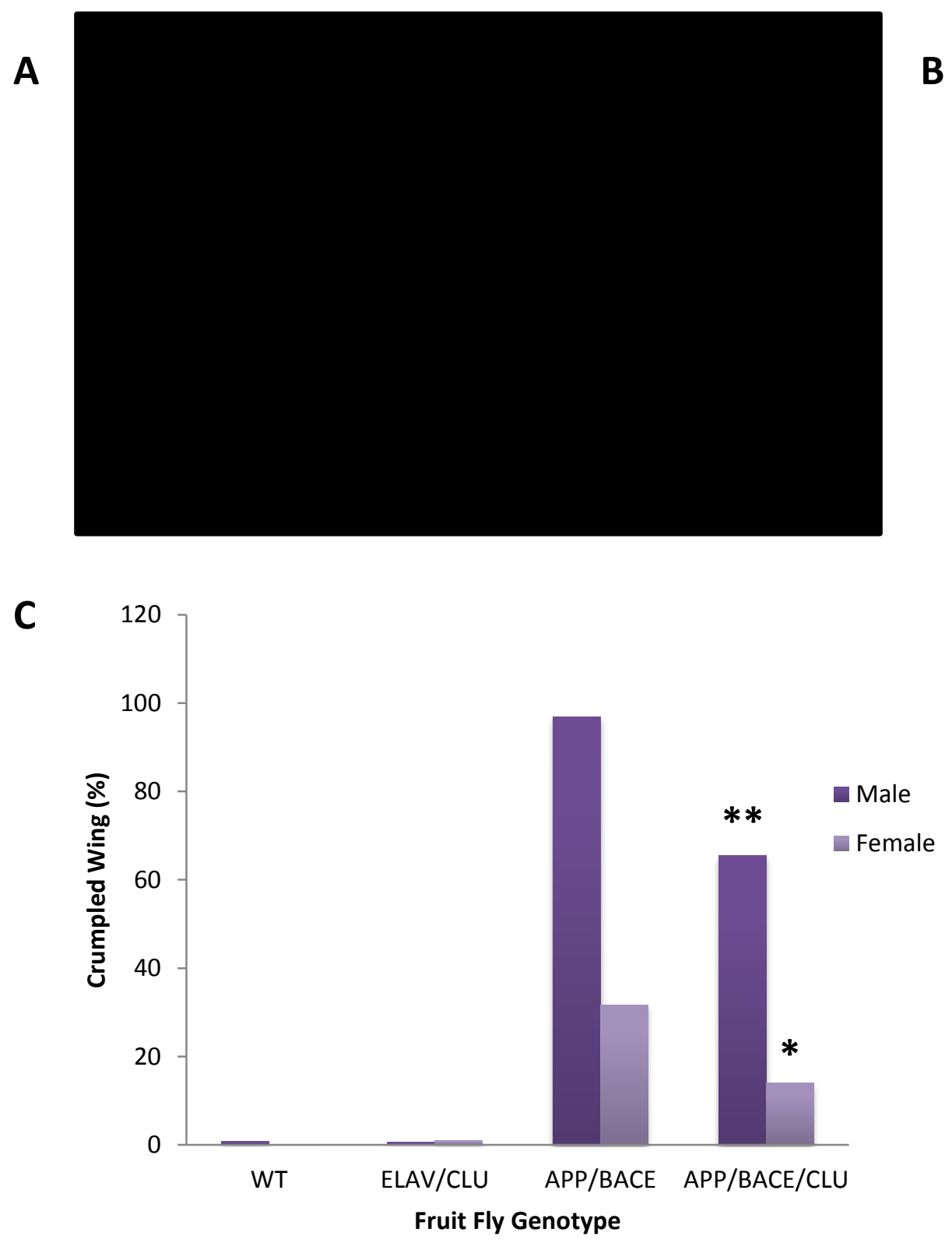

Figure 2.17: Clusterin rescues morphological crumpled wing defect in APP/BACE AD flies (A) Representative wild type fly with fully developed straight wings with absence of melanotic lesions. (B) Representative APP/BACE fly portraying crumpled wing phenotype and melanotic lesions located on the abdomen and proboscis. Fly images taken from Chakraborty et al, 2011. (C) Graphical representation of crumpled wing phenotype within males and females. (WT) $n=500$ males, 508 females, (ELAV/CLU) $n=524$ males, 546 females, (APP/BACE) $n=97$ males, 363 females, (APP/BACE/CLU) $n=226$ males, 343 females. * indicates significant difference (decrease in crumpled wing phenotype by $17.7 \%)$ between CLU treated female AD flies compared to untreated APP/BACE flies. ** indicate significant difference (decrease in crumpled wing phenotype by $31.4 \%$ ) between CLU treated male AD flies to untreated APP/BACE flies. 
2.6 Clusterin Reduces Full Length APP and A ${ }_{42}$ levels in SwAPP SH-SY5Y cells but not in the APP/BACE fly

One of the key pathological characteristics of $A D$ is how the full length APP protein (FL-APP) is processed by beta secretase (encoded by the BACE gene) and gamma secretase. Normal regulation of the APP protein in healthy individuals result in FL-APP cleaved by alpha and gamma secretase, resulting in non-toxic levels of APP processed fragments which are rapidly cleared from the body. However in Alzheimer's disease, APP is incorrectly processed by BACE which results in beta $C$ terminal fragments $(\beta-C T F)$ that are further processed by gamma secretase resulting in amyloid beta fragments $A \beta_{1-42}$ with the most prevalent detrimental isoform being $A \beta_{42}$. To examine the effect of CLU on Alzheimer's disease pathology, I measured the levels of $\beta-C T F$, FL-APP and $A \beta_{42}$ in the APP/BACE fly and the SH-SY5Y+swAPP models of Alzheimer's disease. As clusterin is a stress-induced protein, the overexpression of CLU in these models should either reduce the cleavage processes on FL-APP or increase the clearance of $A \beta_{42}$.

Using Western blot analysis of SH-SY5Y cells, there is a $48.79 \%$ higher abundance of FL-APP present $(p<0.001)$ in the SWAPP/CLU ${ }^{+/+}$model (Figure 2.18A-B). This result suggests that there is a decrease in processing of the FL-APP protein by BACE and gamma secretase. This inference is supported in my measurements of $A \beta_{42}$ using ELISA, which indicated a decrease of $~ 30 \%$ between sWAPP $/ \mathrm{CLU}^{-/-}$and sWAPP/CLU ${ }^{+/+}$models $(p<0.001$ ) (Figure $2.18 \mathrm{E}$ ). $\beta$-CTF levels were also examined using Western blot analysis, however since this protein is an intermediate step before cleaved by either gamma secretase or simply cleared through other mechanisms, levels were too low to be detected. This would serve as in indirect link as to how much amyloid accumulation and processing is occurring. Preliminary experiments show that when the SWAPP SH-SY5Y cell line is treated with 10 uM gamma secretase (Compound E) inhibitor $\beta C T F$ levels are detected in strong levels (Figure 2.19). WT levels were not assessed as there isn't any presence of APP within control SH-SY5Y cell lines. 
However upon examining FL-APP levels in the fly model, I did not detect a difference in FL-APP in APP/BACE compared to APP/BACE/CLU (Figure 2.18C-D). In contrast, a dramatic change was detected in a comparison of WT and APP/BACE, wherein there was increase of $94.15 \%$ in APP/BACE compared to WT (Figure 2.18C-D). FL-APP also seems to be detected as a doublet, except at a slightly different size in comparison to SH-SY5Y cells (110-115 kDa compared to $105 \mathrm{kDa})$. This could be due to detection of the fly's natural orthologue of APP (APPL) that is not by BACE due to the absence of the required cleavage domains. Using the commercial ELISA kit that detects human $A \beta_{42}$, levels of $A \beta_{42}$ in the APP/BACE and APP/BACE/CLU flies were below detectable levels (i.e., below the first spot in the standard curve). As APP/BACE genes are of human nature and have been inserted into the genome of the fly, $A \beta_{42}$ should theoretically have been detected. Several extraction methods were trialled with all resulting in a negative outcome. This suggests that BACE has not been sufficiently incorporated into the fly genome. Alternatively, this result may be due to more than BACE considering that models that only overexpress APP (e.g., the SHSY5Y+swAPP cells) still represent AD pathology, albeit weaker than with the additional BACE mutation. 
A

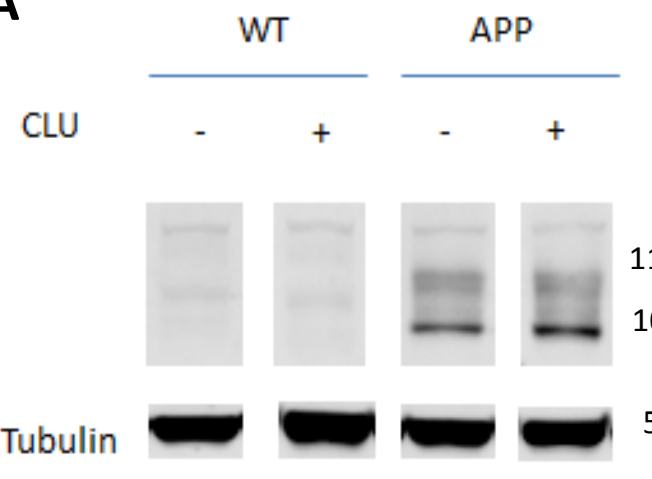

C

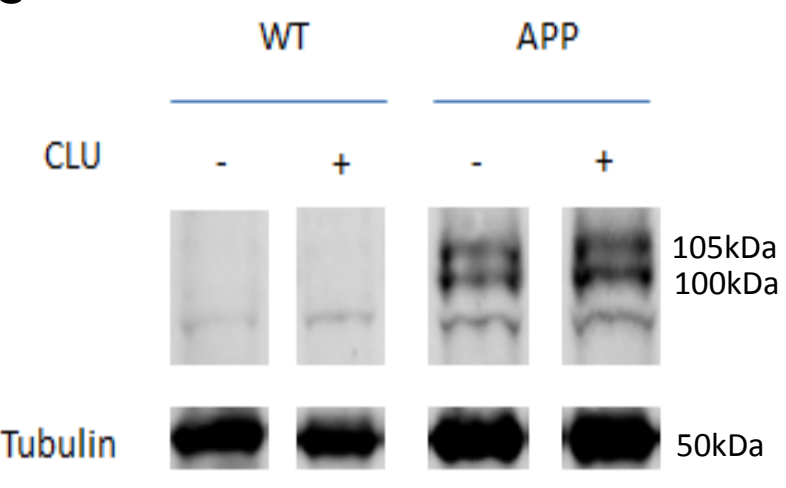

E

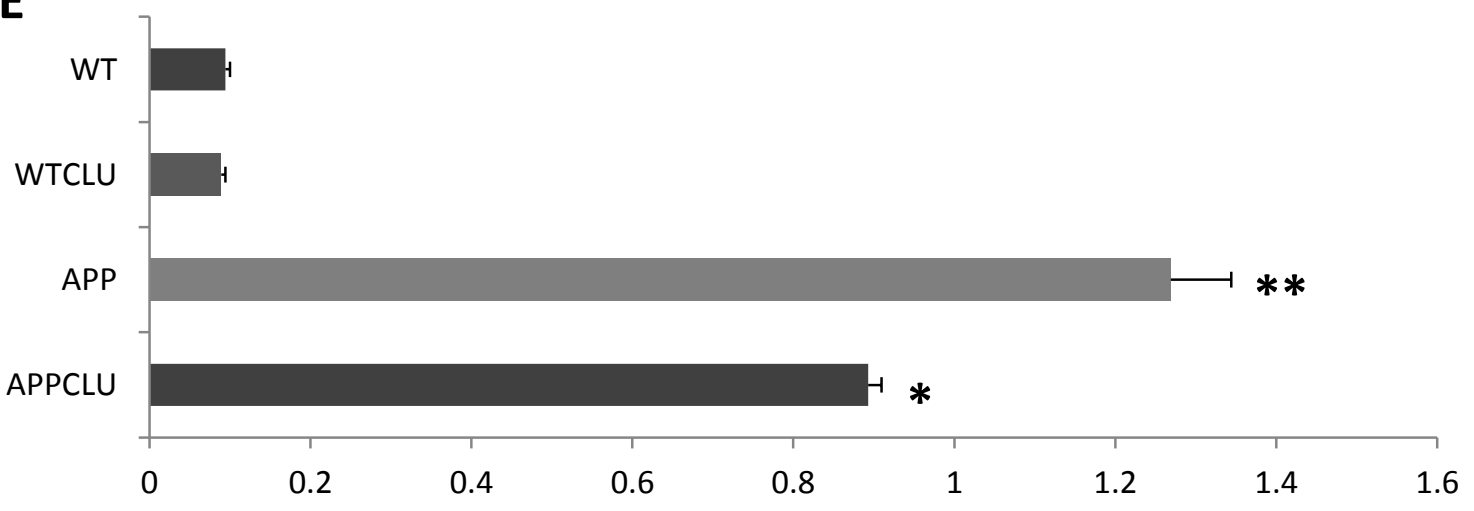

B
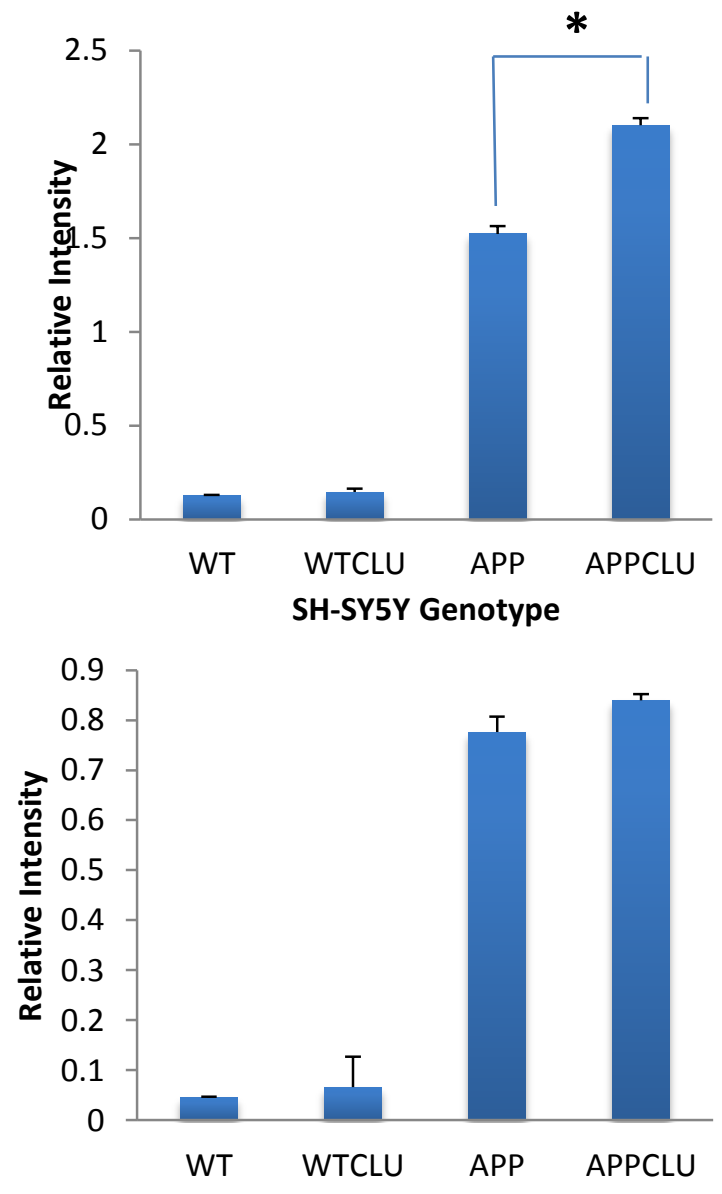

Drosophila Melanogaster Genotype

Figure 2.18: CLU prevents cleavage of FL-APP and $A \beta_{42}$ levels in $A D$ cells and no effect in $A D$ D. melanogaster lines

(A) Representative Western blot analysis showing the effect of CLU overexpression on FL-APP processing in SWAPP SH-SY5Y cell line. $20 \mu \mathrm{g}$ protein loaded $\left(3 \times 10^{\wedge} 5\right.$ cells harvested and protein loaded accordingly). * indicates significant increase of FL-APP in CLU treated AD cells compared to untreated AD cells $(p<0.001)$ (B) Densitometry analysis of $(A)$ showing a $38 \%$ increase in FL-APP protein. (C) Representative Western blot analysis showing the effect of CLU overexpression in APP/BACE drosophila line. $20 \mu \mathrm{g}$ protein loaded (D) Densitometry analysis of (C) showing an absence of FL-APP processing in APP/BACE flies. (E) A ${ }_{42}$ ELISA analysis of SHSY5Y/sWAPP media i.e. excreted $A \beta_{42}$ levels. * indicates significant change between CLU treated $A D$ cells compared to $A D$ cell $A \beta_{42}$ level $(p<0.0001)$. ${ }^{* *}$ indicates significance between $W T$ and AD cells $(p<0.001)$. Statistics calculated by Student $t$-test and standard error used. All experiments repeated in triplicate. 
A

SWAPP SH-SY5Y

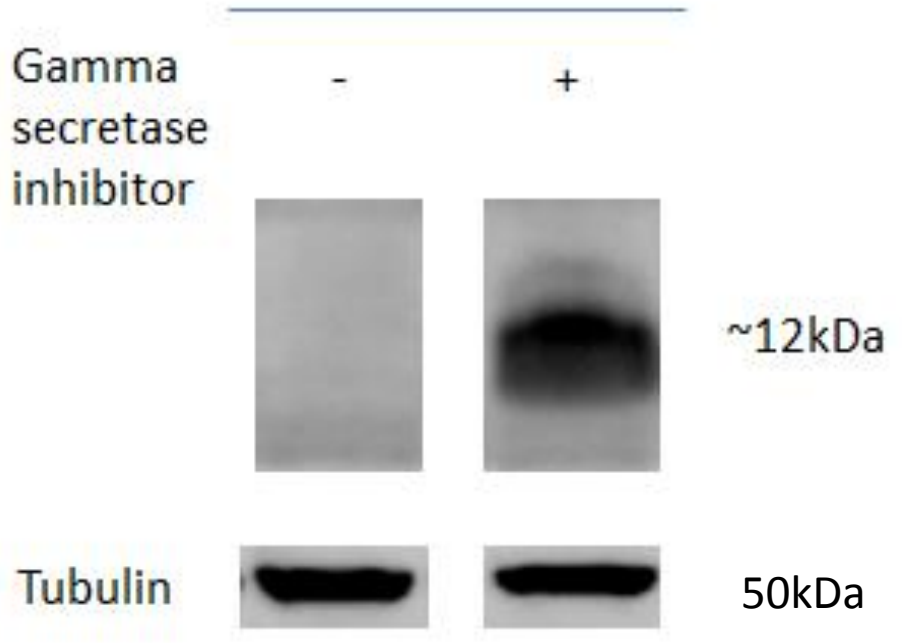

B

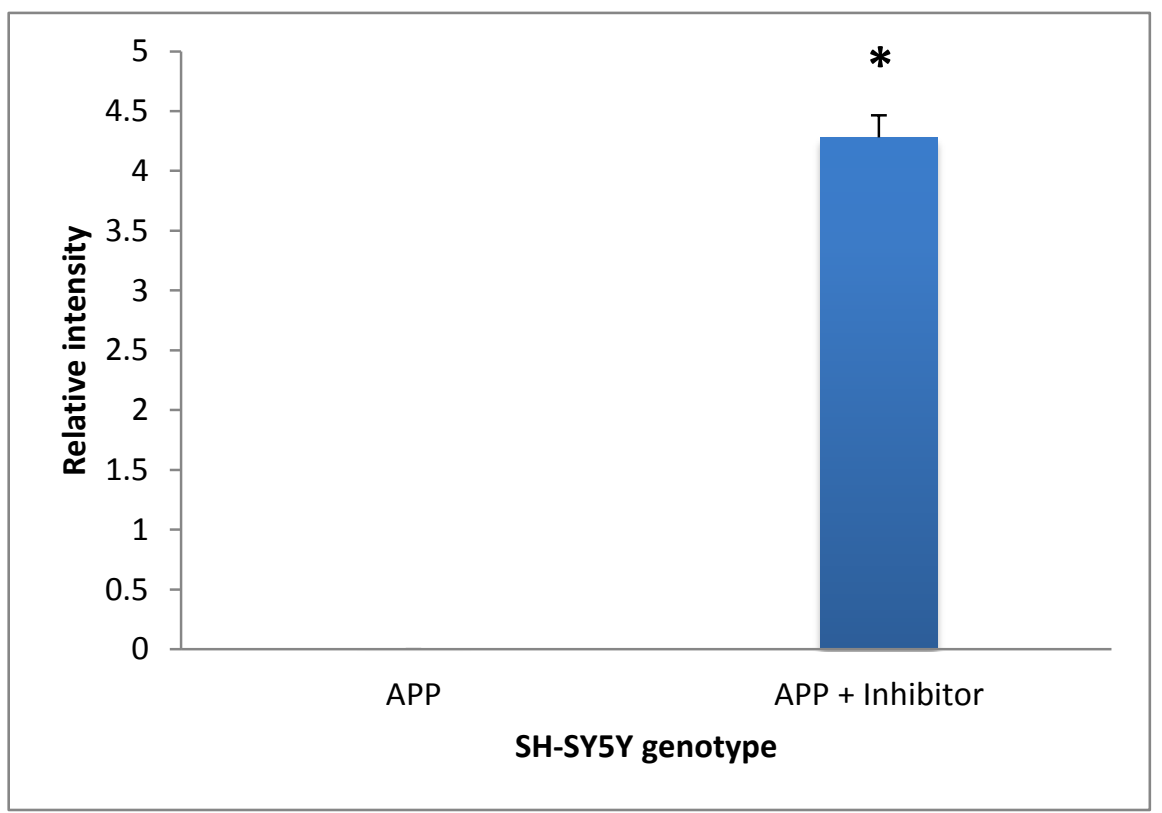

Figure 2.19: Treatment with gamma secretase on SWAPP SH-SY5Y cells improves detection on $\beta C T F$ fragment

(A) Representative Western blot analysis showing the identification of $\beta C T F$ when swAPP SH-SY5Y cells are treated with 10uM gamma secretase inhibitor 20 ug protein loaded. Experiments done in triplicate, $3 \times 10^{\wedge} 5$ cells harvested and protein loaded as indicated. (B). Densitometry analysis of (A) showing significant increase in BCTF detection. Standard error used. * indicates significant difference between gamma secretase treated AD cells compared to untreated $A D$ cells. Student t-test and standard error used, $p<0.0001$. 
2.7 Clusterin Mobilizes Cholesterol Aggregates in the APP/BACE Fly Model of Alzheimer's Disease

A large reservoir of free cholesterol is found within the brain and is one of the key lipids that modify the onset and progression of $A D$. Cholesterol accumulation within brains of patients with $A D$ correlates with amyloid beta plaque formation. Attention was further drawn to changes in cholesterol after it was found that levels of $A P O E$, the number one genetic risk factor for $A D$, is implicated in the transport of free cholesterol through the blood brain barrier and therefore influences cholesterol levels in the brain. Further investigations of APOE and cholesterol also identified a role for CLU in the mediation of cholesterol clearance. With CLU being previously reported having apolipoproteolitic activity as a chaperone protein that also influences cholesterol efflux; I predict that CLU will reduce cholesterol levels in the APP/BACE flies and SWAPP SH-SY5Y cells. I used filipin, a fluorescent stain that binds unesterified cholesterol, to localize free cholesterol in the fly brains (Figure2.20) and human cells (Figure 2.21).

Filipin staining in the APP/BACE fly brain compared to the wild-type brain revealed a $75.1 \%$ increase $(p<0.01)$ in the APP/BACE fly with an increase in cholesterol aggregates with localisation found around the medial middle antennocerebral tract (mACT). The mACT projects directly from the antennal lobe (the first order olfactory neutrophil) to the lateral horn with a subset of fibres directed towards the pedunculus, with synapses projecting towards certain parts of the mushroom body. Filipin aggregates also seem to be consistently found within the medulla. Other images captured seem to lie within these two regions of the brain, however there is widespread detection in which few images show detection of cholesterol detected in the alpha-alpha' region of the mushroom body, the learning and memory centre of the brain. In contrast, the cholesterol aggregates in the APP/BACE/CLU fly reflect the dull staining observed in the wild type fly, with $70.52 \%$ reduction $(p<0.01)$ of cholesterol in APP/BACE/CLU flies compared to the APP/BACE flies. The observation of a 
particular loss of cholesterol aggregation was variable and hard to pinpoint, however the medulla region of the brain seemed to still contain low levels of cholesterol aggregation, whereas towards the centre of the brain, staining was more diffuse. It should be noted that the sample size is relatively small for all $(n=$ 8), thus these results should be taken with caution as experimental repeats would be recommended to have full confidence in these findings.

In analyses of human cells, filipin fluorescence was increased $(p<0.01)$ in sWAPP SH-SY5Y cells compared to WT SH-SY5Y cells (Figure 2.21A-B top right panels). To link autophagy and cholesterol homeostasis, I treated cells with bafilomycin, a vATPase inhibitor that disrupts autophagic flux, and I observed a further increase in cholesterol levels (Figure 2.21A-B bottom panels). Due to time constraints, SH-SY5Y cells transfected with $\mathrm{CLU}$ were not visualised. Characterisation of these cells is crucial for characterizing the effect of CLU on cholesterol homeostasis and further testing the results described above in the fruit fly. 
A
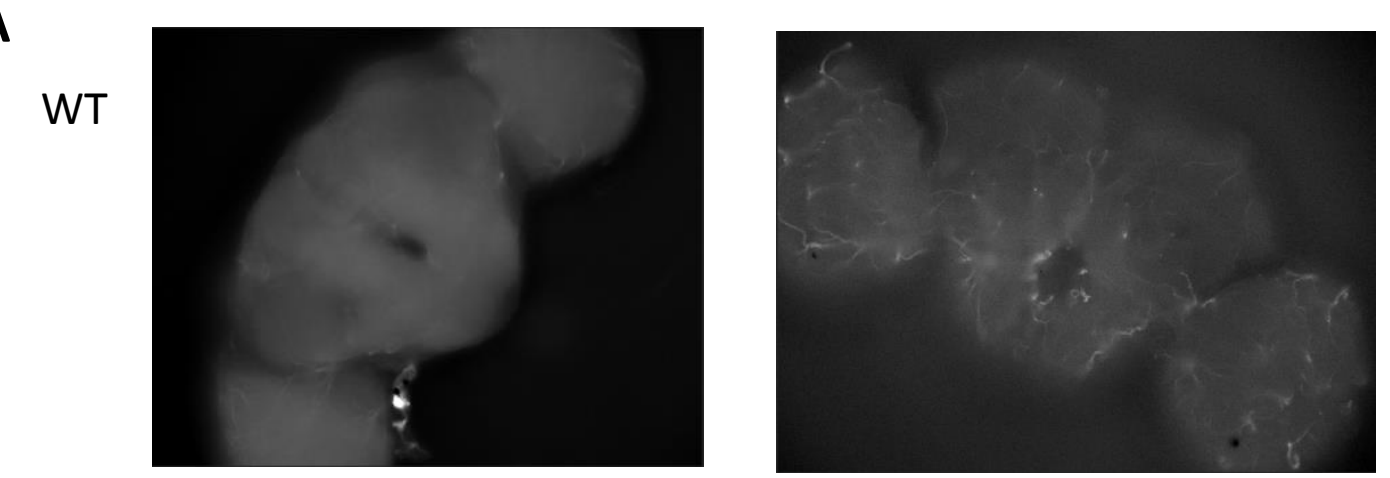

\section{ELAV/}

CLU
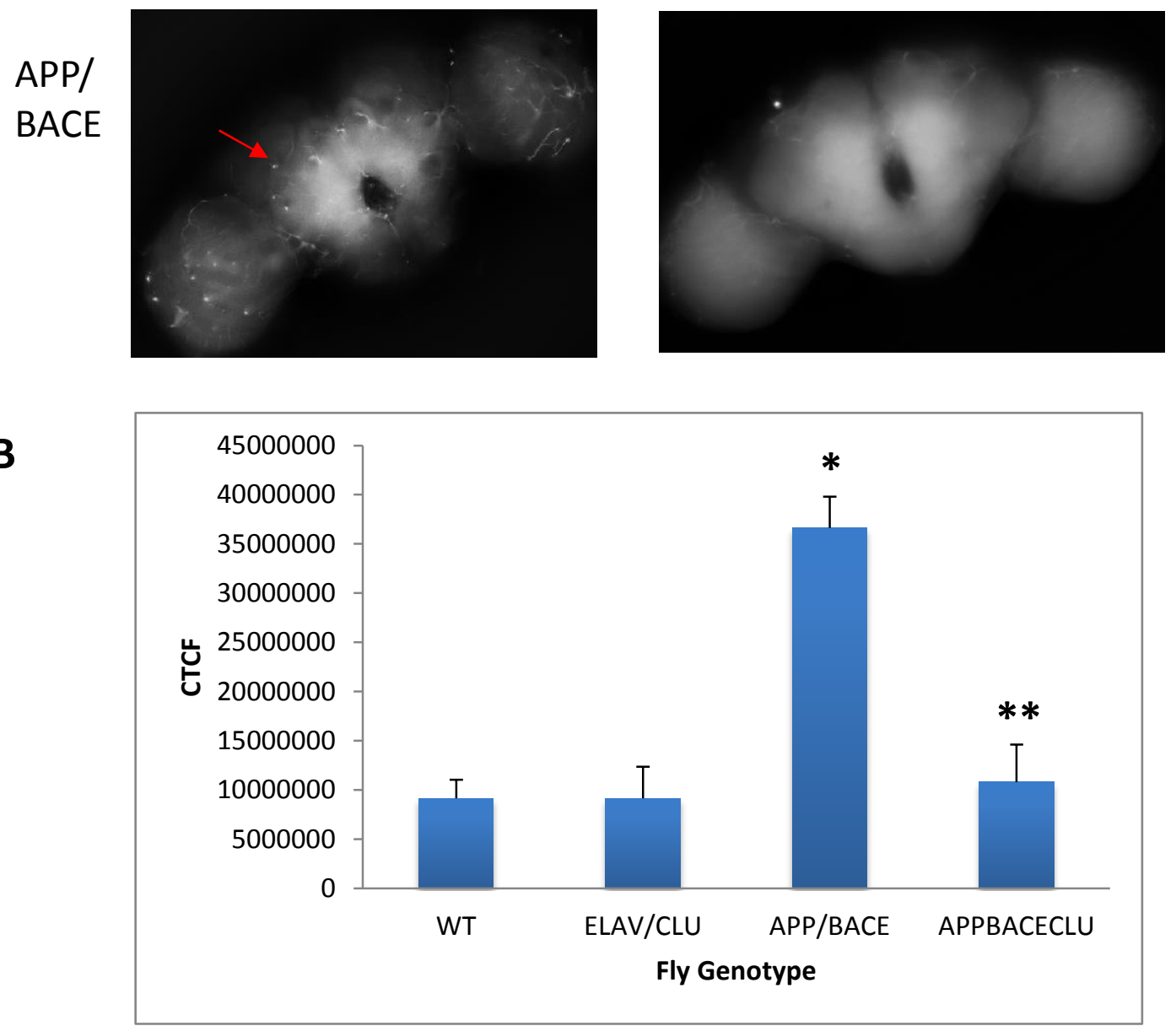

Figure 2.20: Filipin detection reveals decreased free cholesterol content within APP/BACE/CLU fly brains

(A) Filipin staining observations using Olympus BX63 microscope operated to examine WT, APP/BACE (AD fly), APP/BACE/CLU, and ELAV/CLU flies. Cholesterol aggregates found in APP/BACE in comparison to WT fly, localised mainly at the $\mathrm{MACT}$ region (red arrow) whereas clusterin treated flies have a significant reduction in overall cholesterol content. (B) Graphical representation of cholesterol levels (all $n=8$ ). ${ }^{*}, p<0.01$, student $\mathrm{t}$-test, comparison between WT and AD flies. ${ }^{* *}, \mathrm{p}<0.01$, Student $\mathrm{t}$-test, comparison between $A D$ flies and $A D$ flies with $C L U$ overexpression. Standard error is used. (B) Statistical analysis (Image J software) of fluorescent levels of (A). Standard error is used. Analysis conducted by measuring Corrected Total Cell Fluorescence (CTCF) by Image J software. CTCF $=$ Integrated density - (Total area of cell $\mathrm{x}$ mean fluorescence of background). 

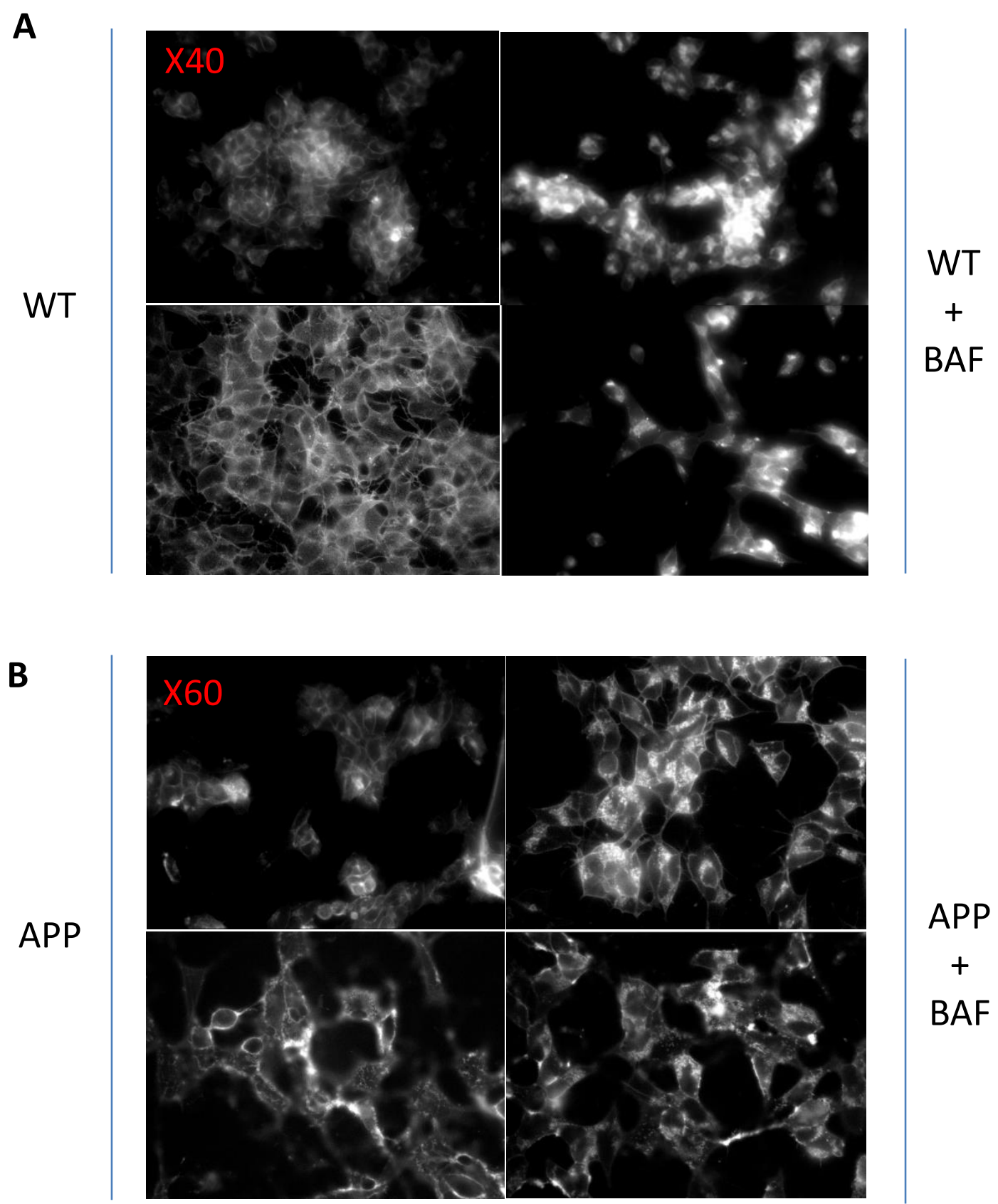

Figure 2.21: Filipin detection reveals increased cholesterol content within SWAPP SH-SY5Y cells

(A-B) Filipin staining on Olympus FV-1000 confocal microscope within WT and APP/BACE fly with and without bafilomycin under (A) 40x and (B) 60x magnification. Visual analysis portrays cholesterol aggregates being more dominant in SWAPP cells in comparison to WT cells, as well as cholesterol content is further accumulated in bafilomycin treated cells; $n=10$. 
2.8 Clusterin May Reduce Cholesterol levels in the Brains of the APP/BACE Fly Model of Alzheimer's Disease

To further investigate the effect of CLU on cholesterol metabolism, I measured cholesterol in fly brains. MALDI-TOF mass spectrometry was carried out on lipids extracted from $~ 50$ fly heads (per each genotype). All samples were analysed in triplicate and normalised from background readings detected with solely the 2,5-Dihydroxybenzoic acid (2,5 DHB) matrix. Although there are several isotypes of cholesterol detected by mass spectrometry, the most prevalent peak was peak $369 \mathrm{~m} / \mathrm{z}$. Preliminary results found at $369 \mathrm{~m} / \mathrm{z}$ peak (Figure 2.22) reinforce results shown in the filipin experiments, wherein there is reduced cholesterol in APP/BACE/CLU compared to APP/BACE flies. However, in order to fully validate these results, an $\mathrm{ms} / \mathrm{ms}$ analysis or a cholesterol standard is required to confirm this peak is indeed cholesterol.

It was my intention to examine the effect of CLU in WT SH-SY5Y and SWAPP SH-SY5Y cells; however my optimal transfection efficiency of around $40 \%$ was not enough to account for variability in mass spectrometry readings between samples. Therefore, in order to increase the percentage of CLU positive cells, FACS sorting was trialled in swAPP/CLU ${ }^{+/+}$cells by using the BD Influx FACS sorter to sort transfected cells (GFP positive) from non-transfected cells (GFP negative)(2.24A). Gating strategies were designed to exclude doublets (GFP positive closely followed by GFP negative cell) (Figure 2.23A), detection of GFP positive cells (Figure 2.23B), detection of viability stain (DAPI) of cells (DAPI penetrates dead cell walls more easily than live cells which will exclude the stain) for percentage of live to dead cell count in sample (Figure 2.23C), and overall percentage of positive GFP/CLU cells in sample (Figure 2.23D). Post sort 1 result procedure resulted in $64 \%$ recovery of total amount of cells (Figure 2.23B) with a total of $1 \times 10^{5}$ cells $/ \mathrm{mL}$. However a second sort was trialled in order to achieve higher purity results. Post sort 2 results increased the purity of the sample from $75.5 \%$ to $85.5 \%$, however only $3.5 \times 10^{4}$ cells were recovered (Figure C). This is less than ideal to achieve a sufficient signal from the MALDI-TOF mass spectrometer, even though the purity level of the swAPP/CLU ${ }^{+/+}$is acceptable. Due to low cell 
numbers collected, SH-SY5Y cells were not analysed, however future directions should point towards transfecting a higher amount of cells and only running cells through one sort run.

A

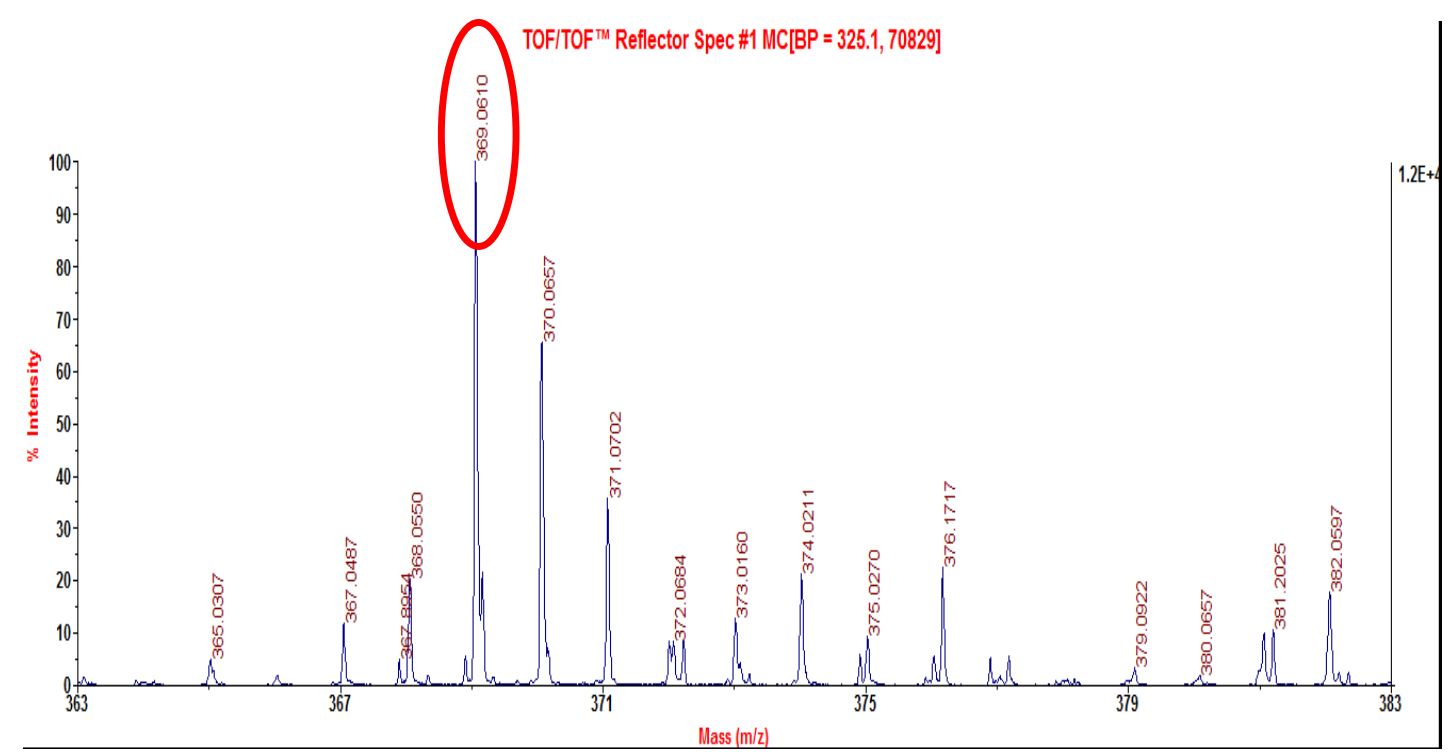

B

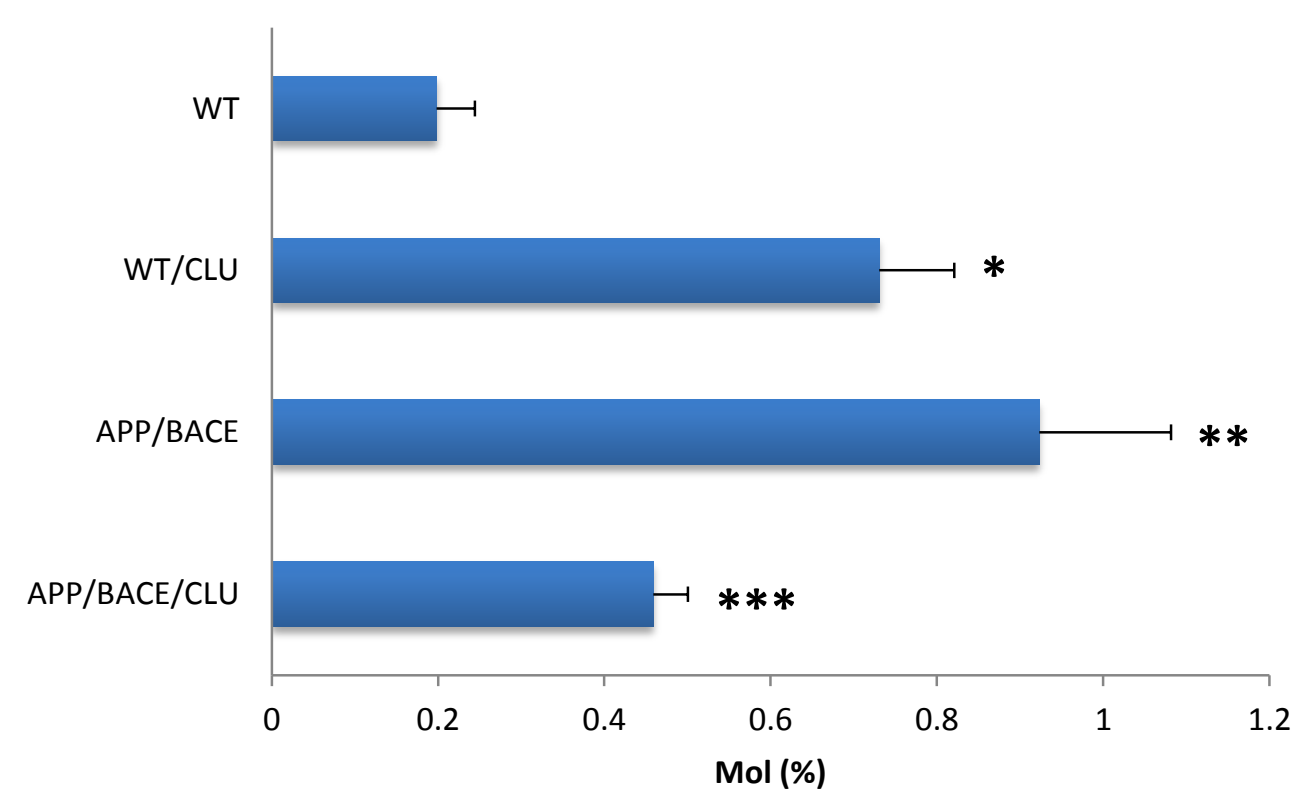

Figure 2.22: MALDI-TOF analysis reveals a decrease in overall cholesterol content when CLU is upregulated in AD flies

(A) Example of peak intensity found in APP/BACE fly $(369.35 \mathrm{~m} / \mathrm{z})$ with intensity of $1.2 \times 10^{4}$. Image analysis observed on DataExplorer ${ }^{\oplus}$ software. (B) Statistical analysis of cholesterol levels found through MALDI-TOF analysis. ${ }^{*}, \mathrm{P}<0.01$, comparison between WT and ELAV/CLU show a 72.89\% increase in cholesterol. ${ }^{* *}, \mathrm{p}<0.05$, comparison between WT and APP/BACE show a 70.82\% increase of cholesterol levels. ***, $\mathrm{p}<0.005$, comparison between APP/BACE and APP/BACE/CLU show a $49.7 \%$ reduction in cholesterol levels. Z-test was used, with standard error. 

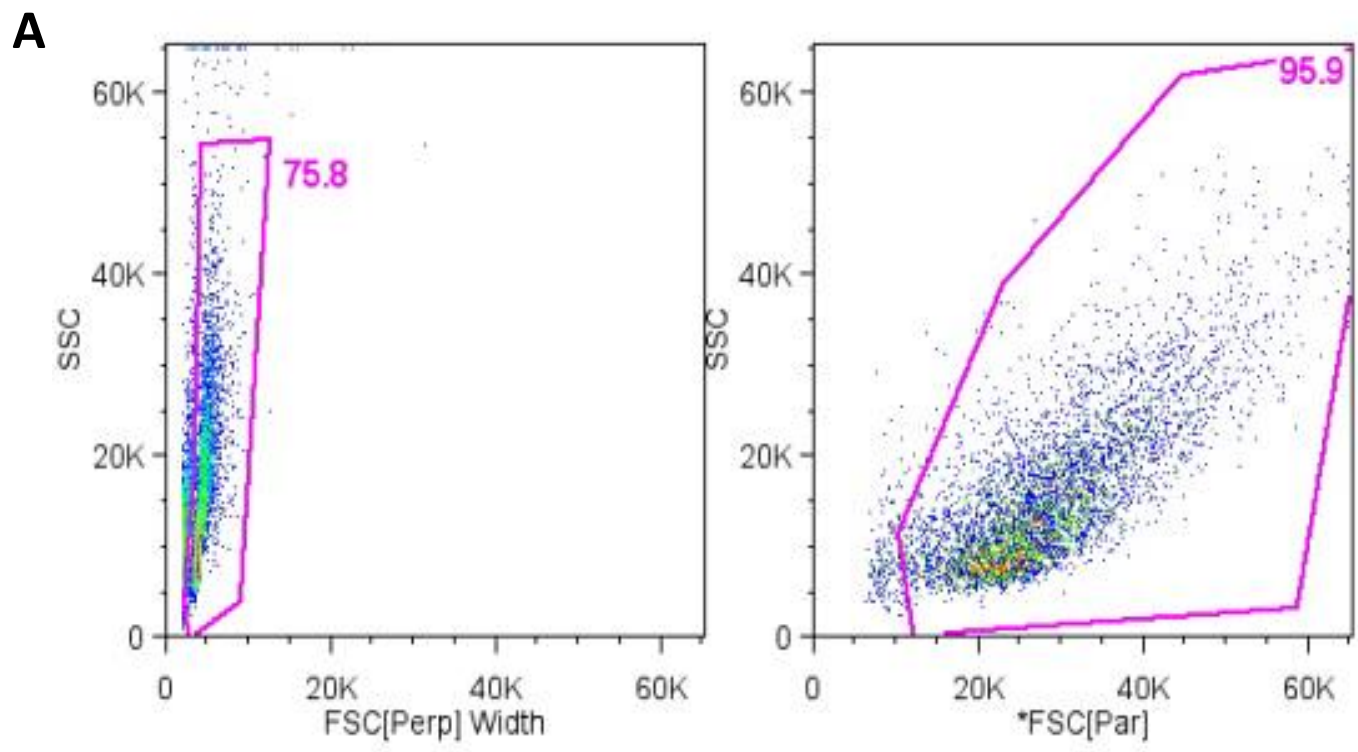

B
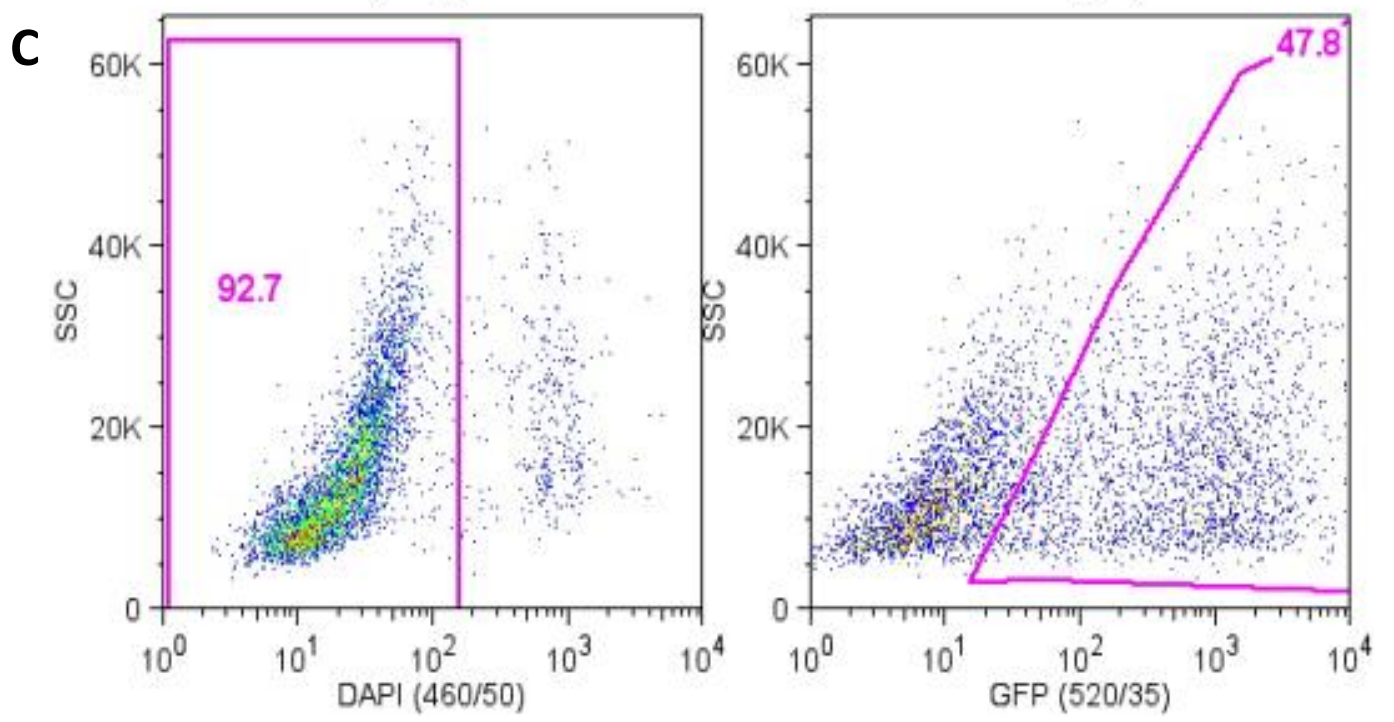

Figure 2.23: Gating strategy for FACS sorting of GFP/(swAPP/CLU $\left.{ }^{+/+}\right)$positive cells (A) Doublet discrimination of cells which are joined together (positive closely followed by a negative GFP cell). $75 \%$ cells recovered from population for single cell detection.(B) Detection of GFP CLU cells (cells of interest). 95.9\% cells detected for GFP signal (C) Detection of viable cells through DAPI negative cells. $92.7 \%$ of cells were viable within GFP positive CLU cell sample (D) Detection of total GFP/CLU positive events in sample. $47.8 \% \mathrm{GFP} / \mathrm{CLU}$ cells were detected (estimated transfection efficiency was $\sim 40 \%$ ). 

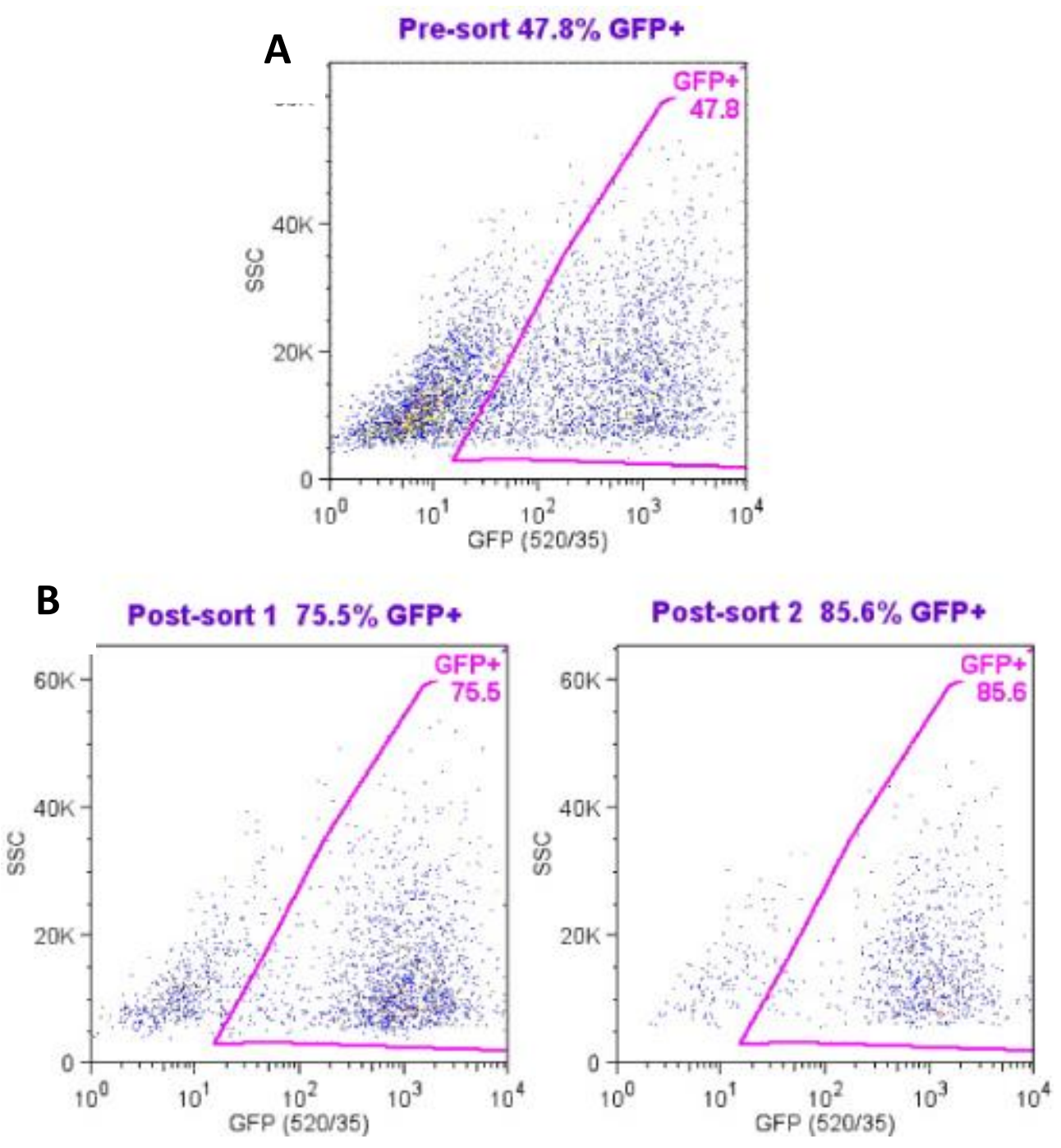

Figure 2.24: Purity check for FACS sorting of GFP/(swAPP/CLU $\left.{ }^{+/+}\right)$cells

(A) Presort results of GFP/CLU positive containing cells. Initial sample of cells contained $47.8 \%$ purity of GFP/ (swAPP/CLU $\left./{ }^{++}\right)$cells. (B)Post-sort 1 of GFP/CLU cells. FACS sorting increased purity of GFP/CLU cells from $47.8 \%$ to $75.5 \%$ (difference of $27.7 \%$ ). (C) Post sort 2 of GFP/CLU containing cells. Post sort \#2 resulted in increased purity from $75.5 \%$ to $85.6 \%$ (difference of $10.1 \%$ ). However significant loss of cells resulted from second FACS sort.

\subsection{Clusterin Disrupts Autophagic Flux in SH-SY5Y+ swAPP Cells}

It was recently reported that CLU enhances cell survival through enhancing lipidation interactions with LC3, allowing it to be conjugated to phosphatidylethanolamide (PE), as well as stabilising LC3-Atg3 interactions that correct dysfunctional autophagy mechanisms ${ }^{1}$. Although this is detrimental to cancer cells, this mechanism would prove beneficial to survival of neurons and prevent degradation of essential memory regions in the brain. In $A D$, 
dysfunctional lysosomal digestion within the autophagic pathway is prominent. As CLU has been shown to aid autophagy, I hypothesise that the overexpression of CLU will correct autophagic processes and aid the degradative processes of defective cargo such as $A \beta 42$ monomers prior to aggregation into senile plaques. To examine the effect of CLU on autophagy, I used Western blot analyses to measure the levels of LC3-II, a protein that is localised on autophagosomal membrane and thus a marker for autophagy. As there is an increase of LC3I-II protein in $A D$ compared to control populations (due to an upregulation of autophagy) we expect increased levels of LC3I-II in SWAPP cells in comparison to WT SH-SY5Y cells. As CLU increases the lipidation of LC3II as well as stabilising the LC3-Atg3 complex to correct dysfunctional autophagic activity, sWAPP/CLU ${ }^{+/+}$ cells should downregulate the overall induction of autophagy, as correction of autophagic processes would downregulate substrate overload in autophagolysosomes.

My densitometry analysis of LC3I-II levels between wild type and SWAPP cells showed there was a significant decrease of total LC3 (LC3I-II) protein levels by $44.65 \%$ in swAPP/CLU ${ }^{+/+}(p=0.01)$ when compared to swAPP/CLU ${ }^{-/-}$(Figure 2.25A-B). Bands detected from western blot were normalised to alpha-tubulin. To further decipher and potentially enhance this result, I treated all cells with bafilomycin, an inhibitor of the VATPase- $\mathrm{H}^{+}$pump in the autophagy pathway that controls lysosomal acidification levels. The bafilomycin treatment resulted in increased detection of LC3-II protein within all cell lines (WT SH-SY5Y, SWAPP SHSY5Y and CLU treated cells) (Figure $2.25 \mathrm{C}$ ), as the degradative process by autophagolysosomes was hindered. This enhanced signal increased the signal to confidently decipher the effect of CLU in that bafilomycin-treated SH-SY5Y+CLU cells had 23\% ( $p<0.01$ ) less LC3-II than bafilomycin-treated swAPP SH-SY5Y cells (Figure 2.25E). Results from this assay reflect differences shown between swAPP/CLU ${ }^{-/-}$and swAPP/CLU ${ }^{+/+}$with a decrease in $21.34 \%(p=0.01)$ (Figure 2.25E). As previous studies recommend that to correctly extrapolate data for autophagy mechanisms through LC3 detection, inhibitors such as bafilomycin need to be considered alongside LC3 levels without inhibitors ${ }^{155}$, results from our Western blot analyses indicate CLU is regulating autophagy in SH-SY5Y cells. 
A

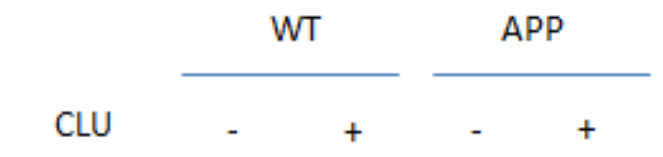

Bafilomycin

LC3I
LC3II
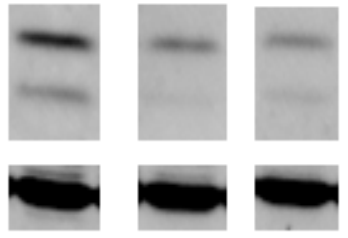

$16 \mathrm{kDa}$

Tubulin
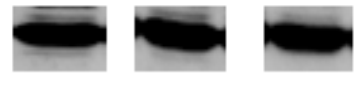

$14 \mathrm{kDa}$

$50 \mathrm{kDa}$

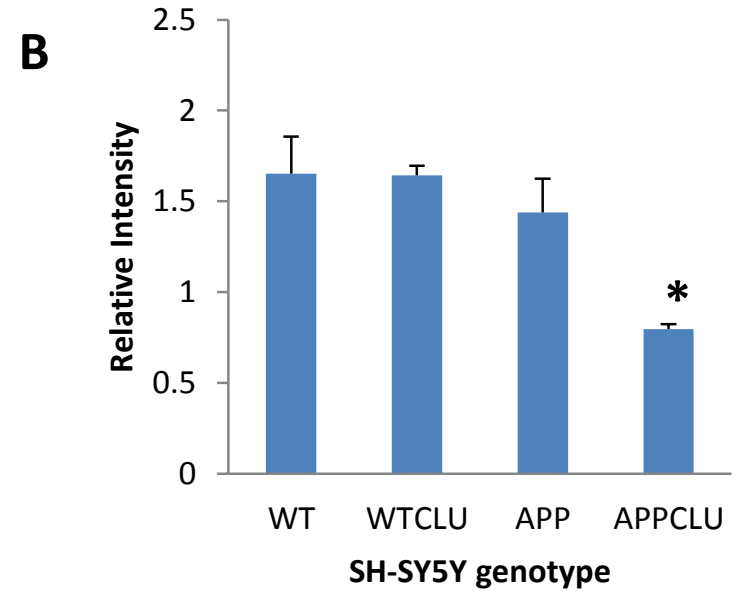

C

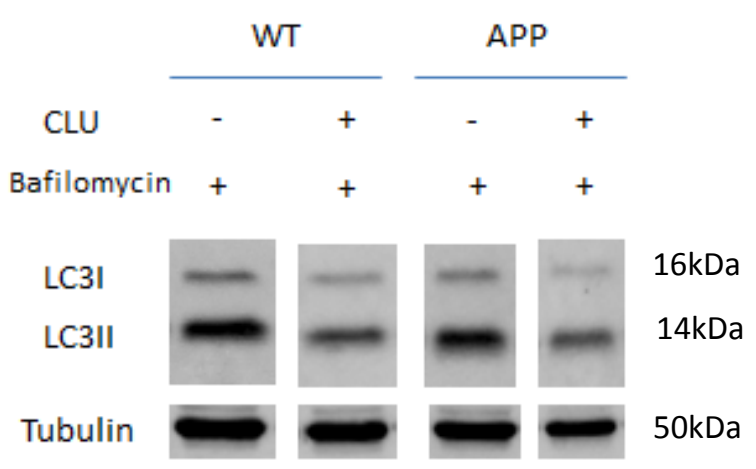

D

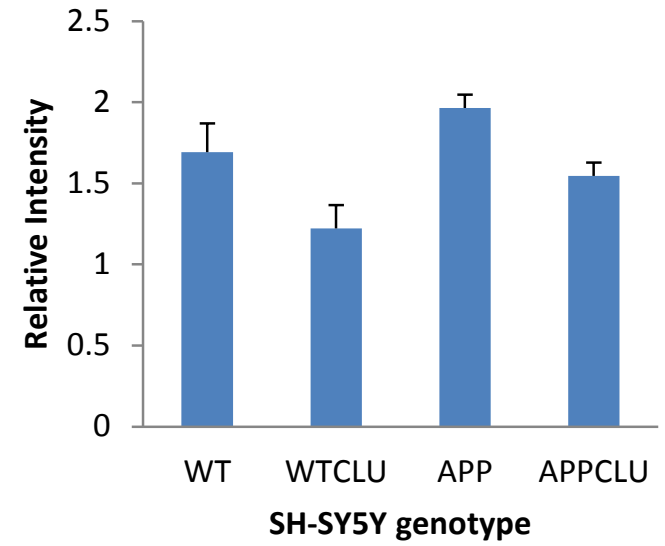

E

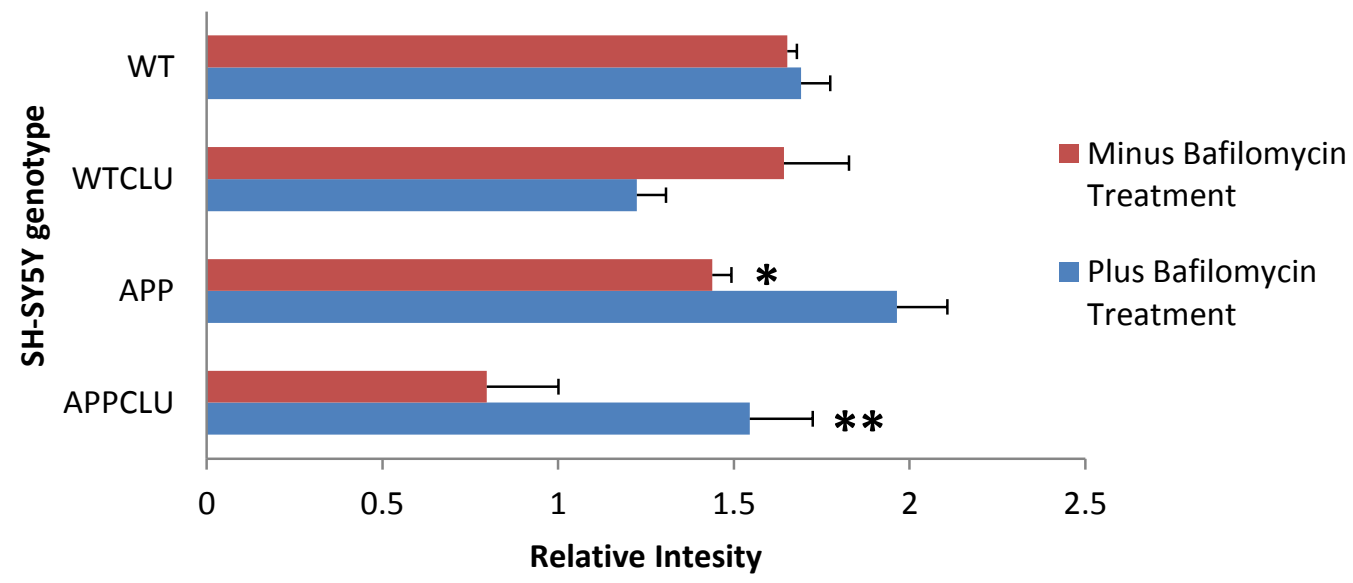

Figure 2.25: Clusterin aids Iysosomal digestion and decreases autophagosome accumulation in AD SH-SY5Y neuroblastoma cells

(A) Representative Western blot analysis showing the effect of CLU on LC3I-II processing in SH-SY5Y/ swAPP SH-SY5Y cell line. (B)Densitometry analysis of (A) showing CLU exhibiting a $44.65 \%$ decrease in overall LC3 expression within SWAPP SH-SY5Y cells, *, p < 0.01, Student's t-test, standard deviation used (C) Representative Western blot analysis showing the effect of CLU on LC3I-II processing in SH-SY5Y/swAPP SH-SY5Y cell line treated with bafilomycin for 24hrs. (D) Densitometry analysis of (C) showing CLU exhibiting an effect on WT and swAPP SH-SY5Y cells by reducing LC3I-II by $23 \%, *, p=0.01$, Student's t-test, standard error used. (E) Comparison of LC3I-II levels between cells treated with and without bafilomycin. 
2.10 Lysotracker red staining reveals overexpression of clusterin results in visual change but no statistical change lysosomal acidification levels within SWAPP SH-SY5Y Alzheimer's disease cell model

To further test my finding that CLU restores autophagy, I used Lysotracker Red, a fluorescent dye that stains acidified organelles. This particular dye at neutral $\mathrm{pH}$ is only partially protonated due to being conjugated to a weak base. This results in the dye permeating cell membranes. Using this probe to visualise lysosomal acidification, this technique is both qualitative and quantitative analyses of the autophagic process. Utilizing Lysotracker Red in live cell image analysis, SWAPP SH-SY5Y cells show that there is a definite upregulation of lysosomal components by $80.1 \%(p<0.01$ ) (Figure 2.28) shown by red punctate within the cell (Figure 2.26B) in comparison to wild type SH-SY5Y cells (Figure 2.26A). This phenotype is further established when cells are administered bafilomycin for $24 \mathrm{hr}$ within swAPP cells (Figure 2.27), with a $27.2 \%$ increase of Lysotracker red defection in comparison to APP untreated with bafilomycin. Upon examination of the swAPP/CLU ${ }^{+/+}$cell line in the absence of bafilomycin treatment, therapeutic effects are not particularly evident, however after administration of bafilomycin, effects of lowering overall autophagy rates in the sWAPP $/ \mathrm{CLU}^{+/+}$cell line are accentuated (Figure 2.28). As bafilomycin increases the stress levels of the cell, this results in higher lysosomal acidification in untransfected cells and lower levels of acidification in overexpressing CLU cells. However, upon statistical analysis of Lysotracker red images, differences between SWAPP $/ \mathrm{CLU}^{-/}$and SWAPP/CLU ${ }^{+/+}$cell lines proved visual observations to be insignificant, as well as bafilomycin treated samples $(p>0.05)$ (Figure 2.28). This is most likely due to the low number of cells counted ( 100 cells). 


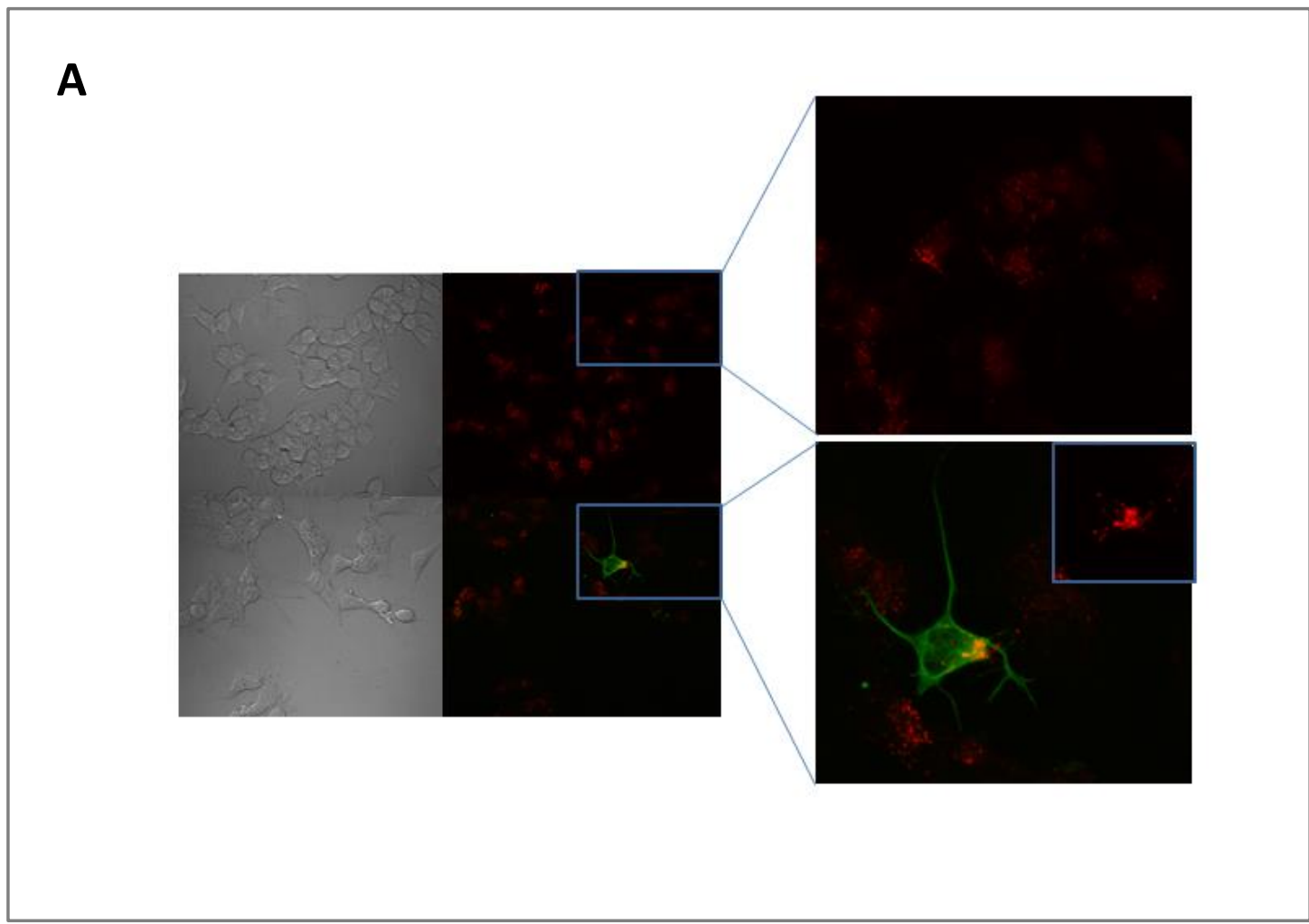

B

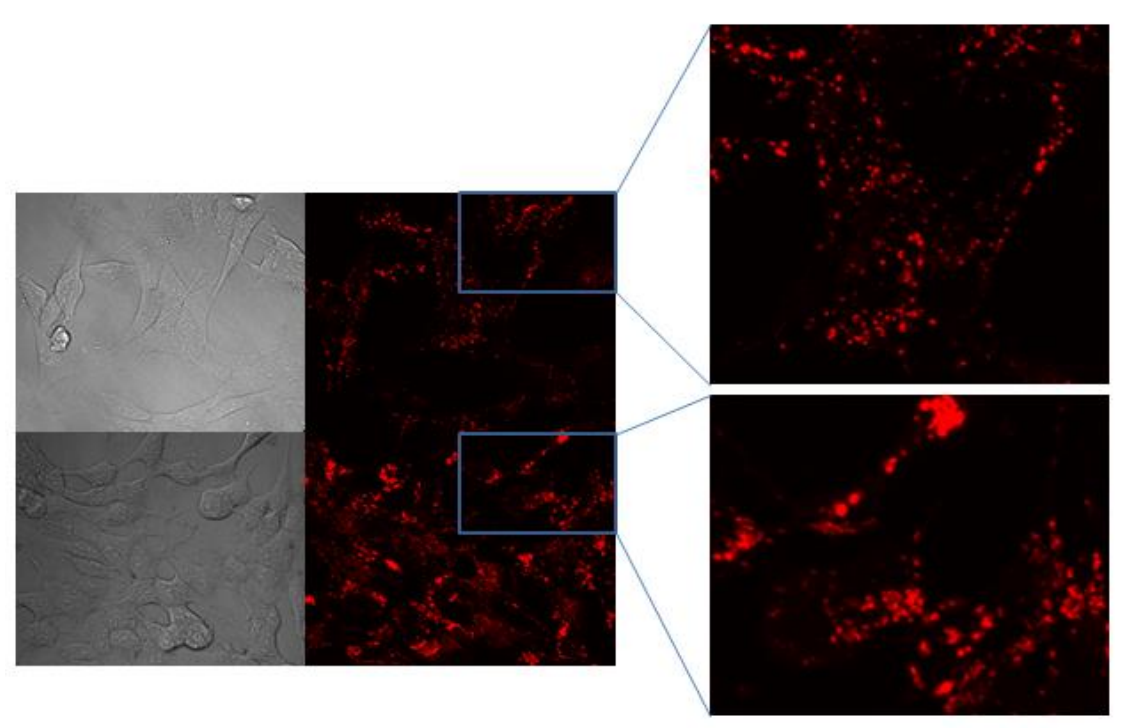

Figure 2.26: Acidification levels are upregulated within SWAPP SH-SY5Y cells in comparison to WT SH-SY5Y cells

(A) Wild type cells (top panels) are dully stained by Lysotracker Red detection of acidified organelles, showing low and effective autophagic processes, where visually WT/CLU expressing cells (bottom panels) suggest upregulation of acidification in autophagosomes. (B) APP cells (top panels) show increased Lysotracker red staining, with APP/BAF cells further increasing this phenotype (bottom panels). 
A

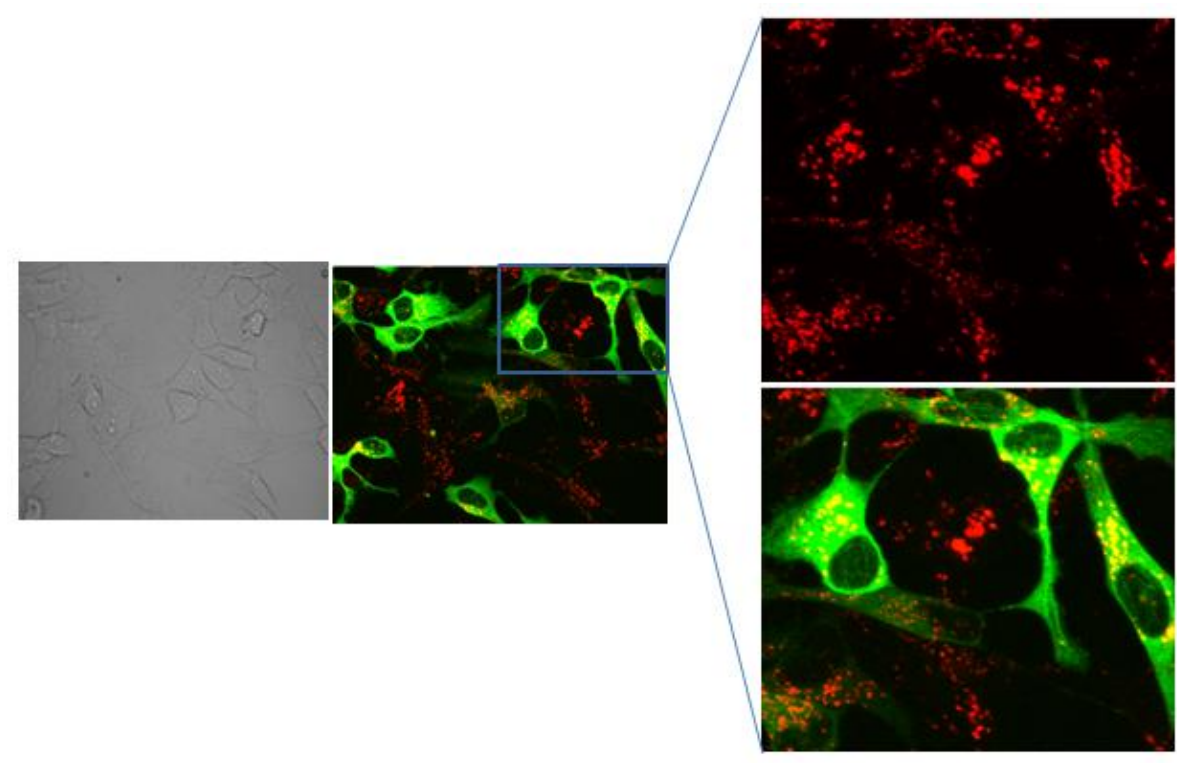

B

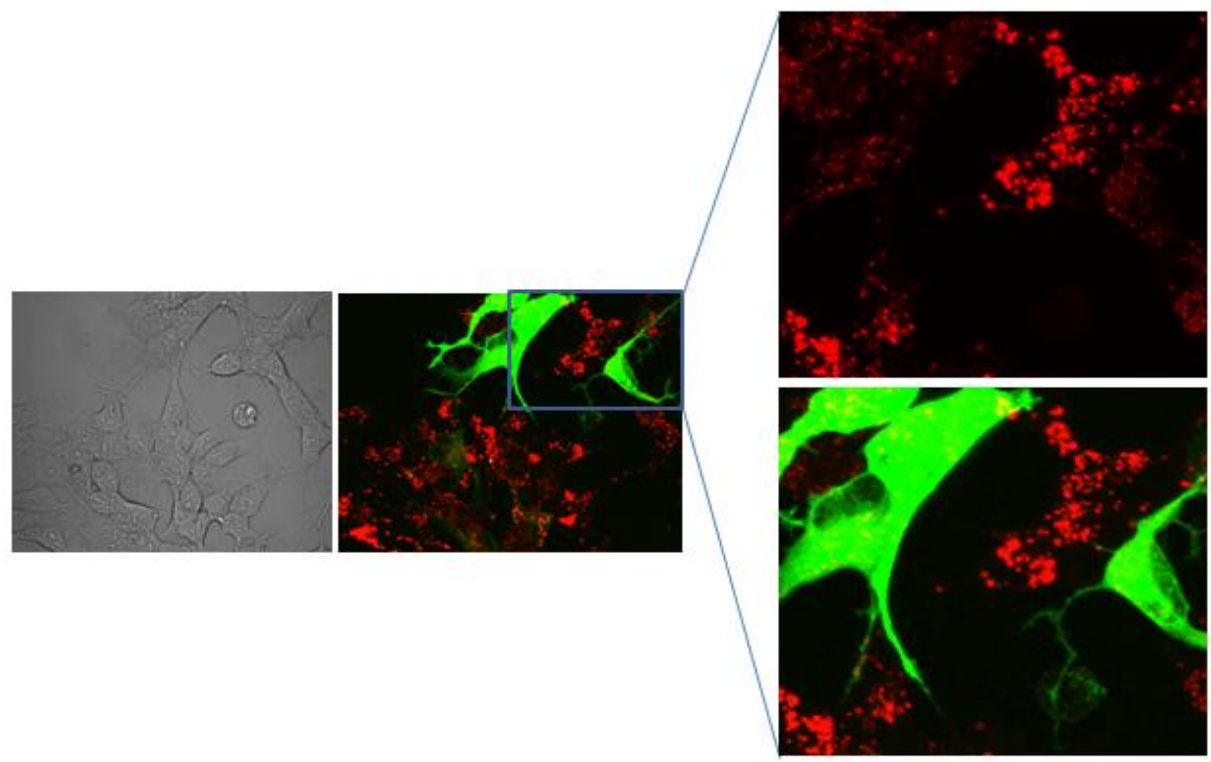

Figure 2.27: Overexpression of clusterin reduces lysosomal acidification levels in a swAPP SH-SY5Y cell line

(A) APP/CLU expressing cells shown inconclusive result as to whether acidification levels are increased or decreased. (B) Bafilomycin treated APP/CLU cells enhance the protective role of $C L U$, as Lysotracker staining is significantly reduced in the APP/CLU transfected cells. 


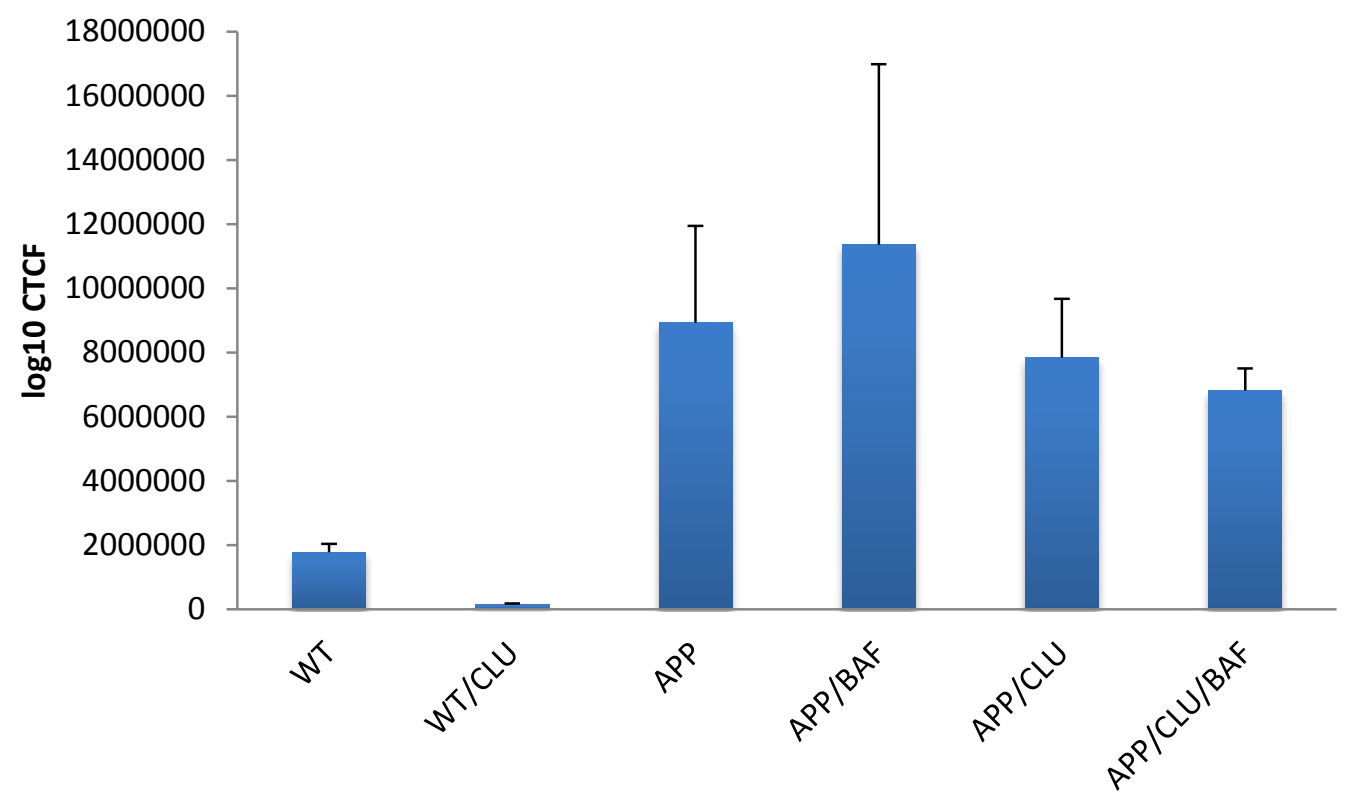

Fly Genotype

Figure 2.28: Quantification of Lysotracker red SH-SY5Y samples

WT-APP genotypes show significant changes whereas APP-APPCLU results prove unchanged. Bafilomycin doesn't show increase of acidification levels. Analysis conducted by measuring Corrected Total Cell Fluorescence (CTCF) by Image J software. CTCF $=$ Integrated density - (Total area of cell $x$ mean fluorescence of background).

Finally, it appears that when CLU is expressed within wild type cells, autophagy levels seem to increase (Figure 2.26A, bottom panel). However statistical validations show that this observation is indeed the opposite, with a decrease in overall autophagy levels by $91.7 \%$ in comparison to wild type untreated $(p=$ 0.01) (Figure 2.28). Student's t-test, standard error used; $n=6$. 


\subsection{DISCUSSION}

\subsubsection{Comparison of in vitro and in vivo models of Alzheimer's disease}

There are various $A D$ models available that have been experimentally utilized for targeting different pathways in order to develop a therapeutic cure for this disorder ${ }^{98,106,107}$. In this thesis, in vitro human neuroblastoma cells (sWAPP SH-SY5Y) and in vivo D. melanogaster (APP/BACE) models have been adopted to investigate the effect of CLU on Alzheimer's disease pathology. For the in vitro model, the swAPP genotype has a double mutation located near the BACE cleavage site on the FL-APP protein ${ }^{108,109}$. For the in vivo model, human APP/BACE genes have been overexpressed within the fly. Mutations in both models lead to increased amyloid beta production ${ }^{105,106}$. Theoretically, the fly would be predicted to be the more powerful model given it contains two mutations that confer $A D$ pathology, while the cells contain just one mutation. Here I used ELISA analyses to demonstrate that $A \beta_{42}$ levels were increased in APP cells compared to WT cells, and subsequently reduced with the expression of CLU (Figure 2.18E). This result provided direct evidence to support the therapeutic potential of activating CLU, as compared to inhibiting CLU that has been previously suggested ${ }^{68,110,111}$. However, the APP/BACE fly model did not corroborate the human cell data in that $A \beta_{42}$ was not detected in the ELISA analyses. As the ELISA kit was designed to quantify human $A \beta_{42}$, combined with my attempts at several extractions that have worked previously with other $A \beta$ ELISA kits ${ }^{112,113}$; this leads us to question whether BACE has been incorporated into the genotype of the fly. The capability of the ELISA kit for detection of $A \beta_{42}$ levels does not appear to be at fault as $A \beta_{42}$ levels were successfully recovered from the supernatant of SWAPP SH-SY5Y cell lines. As the ELISA kit reacts with human products, theoretically any $A \beta_{42}$ levels present within the APP/BACE fly should be detected. Even though the model itself isn't human of nature, the genes expressed in the CNS of the fly are human. Fruit fly models in the past have managed to recover $A \beta_{42}$ levels without encountering this issue, ${ }^{98,114}$ which further supports the idea that BACE has not been successfully incorporated, as $\beta C T F s$ were also not recovered. 
However it was demonstrated within swAPP SH-SY5Y cells that $\beta C T F s$ seem to be present in minute levels that immunoblotting only managed to recover after inhibiting gamma secretase with Compound $E$ which enabled strong detection of $\beta C T F$ levels and showed that $\beta C T F$ was indeed present compared to untreated SWAPP SH-SY5Y cells. Therefore it is possible that if BACE has been successfully incorporated, that $A \beta_{42}$ plaques are being lost through downstream processing, or downstream processing changes the nature of amyloid beta in a form which the $A \beta_{42}$ ELISA kit can't detect. Scientists who have used this Alzheimer's disease model haven't seemed to use this kit before, so perhaps the Invitrogen $A \beta_{42}$ kit works in a manner which can only be used specifically with human samples.

Aside from the difficulty of measuring $A \beta$ in the APP/BACE fly, additional pathology was observed in the development of cholesterol aggregation (Figure 2.20-2.21), decreased survival rates (section 2.5), and defective phenotypical traits such as crumpled wing formation in APP/BACE flies (Figure 2.17A-B). The crumpled wing phenotype was also previously observed in APP/BACE fly ${ }^{98}$. Likewise, the SWAPP SH-SY5Y neuroblastoma cell model was also powerful in demonstrating amyloid metabolism (Figure 2.18A-B), cholesterol metabolism (Figure 2.20-2.22) and autophagy (Figure 2.25-2.28) which were all defective.

\subsubsection{Effects of clusterin on Alzheimer's disease pathology}

CLU's protective roles have also been demonstrated in this thesis within SWAPP SH-SY5Y and APP/BACE AD models. In sWAPP/CLU ${ }^{+/+}$cells, $\mathrm{CLU}$ downregulates FL-APP protein cleavage by $48.79 \%$ ( $<0.01$ ) (Figure 2.18 A-B) as well as decreasing $A \beta_{42}$ levels by $30 \%(p<0.01$ ) (Figure $2.18 \mathrm{E}$ ) which elucidates to CLU exhibiting protective properties upon BACE and gamma secretase cleavage abilities upon FL-APP. As both FL-APP and $A \beta_{42}$ levels have both been affected within swAPP/CLU ${ }^{-/}$and swAPP/CLU ${ }^{+/+}$cells, this suggests that $\mathrm{CLU}$ is not only primarily aiding clearance of $A \beta$ proteins as highlighted in previous reports ${ }^{71,72,115}$, but CLU also exhibits other functionalities which are linked to the protection of FL-APP from being cleaved by BACE and gamma secretase. Note that if observations in SWAPP/CLU ${ }^{-/-}$and swAPP/CLU ${ }^{+/+}$cells resulted in 
insignificant changes in FL-APP protein, but decreased levels in $A \beta_{42}$, the primary role of $A \beta_{42}$ clearance would contribute to previous reports. Further investigations are needed to determine the effect of CLU upon BACE and gamma secretase regulation.

Chaperone properties of CLU are also seen through the decreased levels of overall free cholesterol found in APP/BACE/CLU fly brains by $70.72 \%(p<0.01)$ (Figure 2.20). Administration of CLU in APP/BACE flies also enhanced survival rates as well as reversed morphological defects of the crumpled wing phenotype by $31.4 \%$ in males and $17.7 \%$ in females (Figure 2.17 ). Enhanced survival rates support findings that CLU is implicated in the reversal of ageing processes ${ }^{100}$, having a significant effect more in males than females which suggests that mechanisms underlying ageing are slightly different between sexes. The high survival rate of females in comparison to males could be attributed to different lipid and hormonal regulation between sexes ${ }^{116}$. As males have the prominent role of inducing courtship behaviour, energy resources (such as lipids) are directed towards locomotor activity i.e. distribution of lipids directed to arms, legs and wings of the fly. Therefore as resources are concentrated in these areas, the pathology of APP/BACE is also concentrated to these areas, resulting in a higher mortality rate as there is a defect within transportation of cholesterol and other lipids. Females however play a different role in comparison to males, as energy resources are directed towards reproductive organs ${ }^{116}$. Therefore pathology may affect reproductive organs in comparison to locomotor limbs of the fly which results in a lower mortality rate. CLU acting as a chaperone protein would aid redistribution of lipid to the correct areas and ameliorate defects in APP/BACE flies. There could also be a protective mechanism within the $X$ chromosome itself that aids in survival rates. As flies have two $\mathrm{X}$ chromosomes, female flies would have higher defence mechanisms against pathological defects in comparison to male files which only have one $X$ chromosome.

As CLU is a human protein, flies do not naturally express CLU. However upon induction of CLU within a wild type fly (wcs10), CLU extends the lifespan of the fly by increasing stress resistance against ROS (reactive oxidative species), heat shock, wet starvation and oxidative stress ${ }^{100}$. Within the fly, CLU also 
prevents inactivation of glutamine synthetase (GS) via metal-catalysed oxidation (MCO). GS is an enzyme which (among other areas in the body) is expressed by astrocytes in the brain which production of GS is essential for brain homeostasis $^{117}$. Astrocytes are critical for synaptic plasticity, neuronal functionality by providing metabolites such as lactase and glucose, controls ion environment such as $\mathrm{K}^{+118}$. GS regulation (specifically glutamine-glutamate regulation) in Alzheimer's disease is hindered, as GS expression is significantly lowered, especially around where senile plaques are localised ${ }^{119}$. Impairments of the system in the brain lead to confusion and reduced awareness, as well as changes in behaviour and mood ${ }^{120}$. However upon administration of a sulfhydryl group modifying compound ( $\mathrm{N}$-ethylmaleimide) which blocks the sulfhydral groups of cysteines in CLU, protective anti-oxidant properties against GS inactivation were eliminated ${ }^{100}$.

Oxidised proteins (e.g. MCO) and overall oxidative stress levels occur at a higher rate during the ageing process ${ }^{121} . \mathrm{CLU}$ is also found to be upregulated within normal ageing processes which has led to the discovery of CLU having antioxidant properties ${ }^{122}$ and protein induction is induced to play a protective role against ageing rather than being increased as a consequence of ageing ${ }^{100}$ CLU has previously been described having properties that act in a similar fashion to heat shock proteins (HSPs) ${ }^{123}$ as it acts as a chaperone protein which binds and stabilises misfolded proteins and organelles under environmental oxidative stress $^{124}$. Other similarities between HSPs and CLU include actively operating chaperone mechanisms without the aid for hydrolysis of ATP ${ }^{125}$ as well as both being induced within various diseases which also increase oxidative stress within the body such as Parkinson's disease and Creutzfeldt-Jakob syndrome ${ }^{123}$. In sWAPP/CLU ${ }^{+/+}$cells, $A \beta_{42}$ levels are reduced by $30 \%$ in comparison to sWAPP/CLUI- flies. Just as heat shock proteins operate, $C L U$ binds $A \beta_{42}$ proteins to stabilise the protein and prevent aggregation of $A \beta_{42}$ in senile plaques. Effectively $A \beta_{42}$ will be in a dormant state until other mechanisms such as the autophagy pathway is recruited to recycle and degrade misfolded proteins and organelles. 


\subsubsection{Effects of clusterin on autophagy}

From results obtained (Figures 2.25), we can see through LC3 protein analysis (section 2.10) that CLU downregulates defective autophagic processes in bafilomycin treated SWAPP SH-SY5Y cell line by $21.34 \%(p=0.01)$ in comparison to untreated SWAPP cells. Interpretation of results could lead to suggest CLU would decreases free cholesterol load in the cytosol from being packaged into autophagosomes by transporting them out of the BBB, so there is less stress and misfolded proteins to be packaged and degraded by the autophagy system. To determine if CLU acted upstream or downstream of the autophagy pathway, Zhang and colleagues demonstrated that treatment of rapamycin (an mTOR pathway inhibitor which increases the rate of autophagy) within CLU expressing cancer cells significantly enhanced autophagy rate compared to CLU depleted cells $^{79}$. As SH-SY5Y cells are also cancerous in nature (neuroblastoma cells), findings by Zhang et al suggests that within swAPP SH-SY5Y cells, CLU is first recruited in response to oxidative stress and ROS levels, which downstream effects recruits an upregulation of autophagy once misfolded proteins such as $A \beta_{42}$ have been stabilized by CLU. This in turn aids the lipidation of LC3-PE and stabilizes the LC3-Atg3 complex by shielding $A \beta_{42}$ from directly interacting with autophagosomes, which enables correct autophagy acidification and lysosomal processing. It is important to note that findings by Zhang et al were not replicated in control WT SH-SY5Y cells. However the autophagy mechanism could be cell-cell dependant, as PC3 cells (prostate cancer cells) were examined, whereas cells used in this study are neuroblastoma cells. Other factors that could contribute to differences in findings is the increased passage number of WT SHSY5Y cells used (>P20) when cells were treated for mycoplasma over a course of 2 months.

Interest in the mTOR pathway and the ability of rapamycin to inhibit this pathway is of interest as this pathway is greatly affected in ageing processes ${ }^{126,127}$. Increased lifespan through administration of rapamycin has been shown in Drosophila ${ }^{128}$, C. elegans ${ }^{129}$ and mice ${ }^{130}$. Anti-ageing properties of rapamycin include anti-inflammatory effects exhibited in atherosclerosis ${ }^{131,132}$. 
Alzheimer's disease experiments using rapamycin also showed potential therapeutic effects as administration of rapamycin inhibited the degradation of cognition and memory ${ }^{133,134}$. It was also discovered that rapamycin treatment in 3XAD-Tg mice lowers the accumulation of Tau levels, amyloid beta as well as amyloid beta fibrillary aggregates by $\sim 40-50 \%{ }^{135,136}$ with similar symptoms shown in hAPP (J20) mice after treatment with rapamycin over several months ${ }^{133}$. Clinical trials of drugs that upregulate autophagy such as Latrepirdine ${ }^{137}$ also inhibit the mTOR pathway, whereas polyphenols such as Reveratrol ${ }^{138}$ and Metformin ${ }^{139}$ act to upregulate autophagy through alternative processes such as AMPK activation ${ }^{1}$. As age-related problems include neurodegeneration (e.g. dementia), it can be said that through upregulating autophagy, the ageing process is attenuated.

Studies have not linked how CLU acts in response to these drugs, however it can by hypothesised that since all these drugs directly act upon the mTOR pathway, CLU will behave in the same manner as it does in response to rapamycin, and will recruit to sites of oxidative stress and recruit the necessary amount of autophagy needed to process misfolded proteins and organelles. If this statement holds true and is activated by mTOR drug inhibitors, the finding that CLU decreases abnormal autophagic processes within an AD model would support therapeutic effects exhibited by CLU.

\subsubsection{Effects of clusterin on cholesterol metabolism}

The cholesterol efflux property of CLU was hypothesised to decrease abnormal cholesterol build up in the brain. I demonstrated this with filipin and mass spectrometry analyses within APP/BACE fly brains. APP/BACE fly brain revealed a $75.1 \%$ increase $(p<0.01)$ in cholesterol in comparison to control WT fly brains (Figure 2.20A, left panel), which is supported by mass spectrometry analysis, showing an increase of cholesterol in APP/BACE fly brains by $78.6 \%$ $(p<0.01)$ in comparison to control WT fly brains (Figure 2.21B). Overexpression of $\mathrm{CLU}$ in $\mathrm{AD}$ flies (APP/BACE/CLU) in turn decreased overall cholesterol levels by $70.52 \%(p<0.01)$ (Figure $2.20 \mathrm{~A}$ bottom panel). Mass spectrometry analysis also supports this finding with APP/BACE/CLU cholesterol levels reduced by $50.3 \%$ ( $p$ 
$<0.05$ ) (Figure 2.21). Even though comparing statistical analysis from both techniques reveal different levels of reduction in cholesterol, both findings are statistically significant, with a reduction of cholesterol in APP/BACE/CLU flies at least $50 \%$ when compared to APP/BACE flies. As there is a drastic reduction in cholesterol aggregates in APP/BACE/CLU fly brains, it is important to step back and examine the preferential regions of the brain where cholesterol localises at within the APP/BACE fly in order to observe which regions of the brain is impaired due to cholesterol aggregation.

Observations show that cholesterol levels are highly abundant in the APP/BACE fly brain, with cholesterol being widespread throughout the brain apart from the specific localisation towards the mACT. mACT feeds neurosynaptic information from the antennal lobe (primary information site) and connects to the lateral horn with small subset of fibres directed towards the pedunculus and small region of the mushroom body ${ }^{140,141}$. The pedunculus is a region of the fly brain in which Kenyon cell neuron projections are densely found ${ }^{142}$, whereas the lateral horn is a region of the brain that is part of the olfactory system that is responsible for distinguishing and quantifying odours $^{143,144}$. From these odours, the fly can make decisions based on what stimulant is being received ${ }^{143,145}$. As cholesterol aggregates span across the mACT region, it could suggest that signals delivered from the antennal lobe to the lateral horn is hindered or the signal is completely absent. Damage to this region of the brain would result in abnormal courtship behaviour and other survival instincts. The lateral horn has been suggested to reflect the amygdala within vertebrate brains. The amygdala is a part of the human limbic system and abuts the hippocampus through a structure called the uncus ${ }^{143}$. As the amygdala is responsible for emotional awareness, evaluation as well as risk assessment, damage to this region of the brain would result in emotional cues not being registered $^{146}$. Examples include the lack of flight-or-fight mechanism, as well as not registering aggression or fear. Scientists have hypothesised that if the lateral horn is knocked out then this observation would be replicated ${ }^{143}$. However troubles in selectively inhibiting LH function to test this hypothesis still proves to be an obstacle that needs to be overcome to gain confidence in this theory. 
Cholesterol aggregation is also observed in the medulla region of APP/BACE flies. The medulla is involved in interpretation of visual information from the lamina. Defects in the medulla are consistent with findings from Greeves et al, $2004^{147}$ which report defects in the retina in Alzheimer's disease flies. Greeves postulated that this defect is due to putative $\delta$-secretase expression occurring higher in the retina opposed to other brain regions. $\delta$ secretase acts on APP12 residues on the N-terminal where BACE acts upon, which processing results in longer $A \beta_{42}$ fragments ${ }^{98,147}$. The nature of these fragments would therefore have a bigger role in photodegeneration in the retina than other neurodegenerative characteristics affecting memory and other functionalities $^{98,147}$.

In providing a direct connection between cholesterol, CLU and AD pathology, my data further supports the significant efforts in targeting cholesterol to treat $A D$. In order to address cholesterol build-up in $A D$, statins have been employed to inhibit HMG-CoA to reduce cholesterol levels as well as reduction in LDL levels ${ }^{148}$. HMG-CoA is essential for the production of cholesterol in the liver (around 70\%). As well as lowering cholesterol, statins have antiinflammatory effects by the inhibition of inflammatory matrix metalloproteinases, chemokines and cytokines, as well as having anti-oxidant effects $^{149}$. In an AD ABPPswe/PS1dE9 mouse model, it was found that simvastatin increased synaptic plasticity by decreasing amyloid plaque build-up ${ }^{150}$. Atrovastatin and pitavastatin was found to reduce oxidative stress within an $A D$ APP-Tg mouse model ${ }^{151}$. The Rotteerdam observational study followed 6992 participants for nine years whom took statins and results were correlated to a lower risk of Alzheimer's disease compared to controls ${ }^{152}$. These are many examples showing the promising therapeutic benefits that statins implement within AD patients. The role of CLU was not characterised in these studies, though it would be interesting to elucidate whether CLU is upregulated in response to statin treatments.

It is important to note that the usage of statins not only lowers cholesterol but also affects the autophagy pathway. In macrophages, simvastatin induces therapeutic affects by inducing autophagy through increasing oxidised 
low-density lipoprotein, increasing conversion of LC3I to LC3II (LC3-PE lipidation) and lowering cholesterol accumulation ${ }^{29}$. In $A D$, the presence of abnormally high amounts of cholesterol (as well as mutant beta-APP) upregulates Rab5 and Rab7; two proteins which are implicated in autophagy and are located to early endosomes which downstream effects lead to the overloading of substrates such as lipids and other cargo within late endosomes, as endocytic activation events are accelerated. This results in increased trafficking of lysosomes towards early endosomes, resulting in dysfunctional late endocytic clearance. ${ }^{105}$ Upregulation of Rab5 and Rab7 also transports the vATPase to endocytic vesicles which increases acidification levels of lysosomes.

\subsubsection{Proposed mechanism of action of CLU in Alzheimer's disease}

Based in my results, I propose that within an AD model (Figure 3.1A); free CLU is not present in sufficient levels to counteract the accumulation of misfolded proteins and free cholesterol which are packaged in autophagolysosomes. Reduced CLU levels result in insufficient levels of lipidated CLU to bind and stabilize $A \beta_{42}$ aggregates and transport excess cholesterol past the blood-brain barrier (BBB). Due to the inability of CLU to process $A \beta_{42}$ protein, the autophagy system is upregulated and recruited in attempts to recycle and degrade $A \beta_{42}$. However interactions with $A \beta_{42}$ plaques affect the ability of autophagolysosmal degradative processes. As autophagolysosomes have packaged $A \beta_{42}$ plaques as well as packaging excess cholesterol present within the environment, autophagolysosomes increase and contribute to $A \beta_{42}$ pathology, as well as increasing $A \beta_{42}$ acidification.

However, when CLU is overexpressed in an Alzheimer's disease model (Figure 3.1B), free CLU becomes lipidated which upon binding of excess cholesterol, it interacts through the lipoprotein receptor-related protein (LRP) receptor and enables the transport of free cholesterol past the BBB. CLU is expressed in sufficient levels to bind $A \beta_{42}$ monomers and stabilise increased misfolding of the protein. This will prevent $A \beta_{42}$ aggregation and senile plaque development. As CLU has stabilised $A \beta_{42}$ monomers, this enables the autophagic 

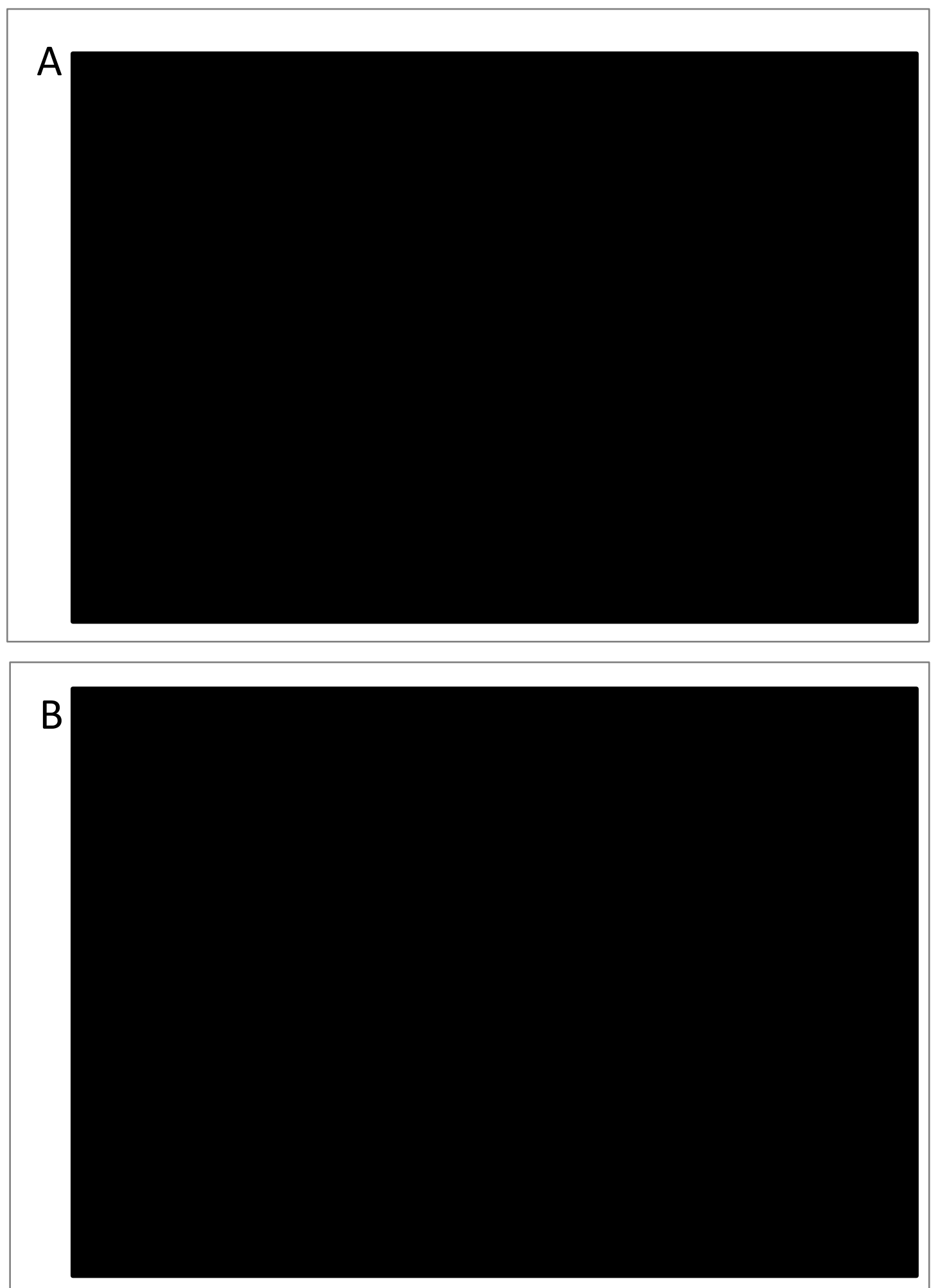

Figure 3.1: Schematic diagram for processes occurring in an AD model

(A). In an AD model, excessive accumulation of free cholesterol and amyloid beta hinders several processes within the cell including increasing acidification levels, and insufficient degradation of misfolded proteins, organelles and other cargo etc which leads to an accumulation of free cholesterol and AB42 levels with this mechanism being what causes amyloid plaque accumulation. (B). When overexpression of clusterin is administered, CLU activate in the presence of environmental stress ques. This leads CLU to do two essential processes. 1. Export cholesterol pass the blood brain barrier and aids LC3-PE conjugation. Figure modified from Di Paolo \& Kim, 2011, Nature. 
system to encapsulate $A \beta_{42}$ fragments efficiently. CLU will aid this process in two ways.

Firstly, as CLU has bound $A \beta_{42}$ fragments, CLU will serve as a barrier, as $A \beta_{42}$ can't directly interact with the autophagosomal membrane as it envelopes the $A \beta_{42}$ monomers for degradation. Secondly, CLU will aid the formation of autophagosome by stabilizing the LC3-Atg3 complex as well as aiding in LC3-PE lipidation. This partially restores normal lysosomal acidification abilities to autolysosomes, allowing the vesicles to degrade the cargo to be retaken up by cells. Through these mechanisms, autophagy is efficient in degrading $A \beta_{42}$ monomers. As excess cholesterol has been lowered through increased cholesterol efflux mechanism by CLU, the substrate overload has been reduced. Oxidative stress will therefore be lowered which in turn will lower the recruitment of autophagosomes. 


\section{CHAPTER 3}

\subsection{FUTURE DIRECTIONS}

In this thesis, I have investigated the effects of CLU on the Alzheimer's disease pathology through the evaluation of APP processing, $A \beta_{42}$ levels, cholesterol metabolism and autophagy regulation within the SWAPP SH-SY5Y cell line and in APP/BACE flies. Even though therapeutic results have been concluded within this thesis, additional experiments are necessary to confirm and provide further insights into these findings.

3.1.1 Further Characterisation of the Effect of CLU on Alzheimer's disease Pathology

Within this thesis, the effect of CLU in the in vitro swAPP SH-SY5Y cell model was characterised by detection of FL-APP protein, $A \beta_{42}$ levels and $\beta C T F$ detection. To complement the in vitro analysis, the in vivo APP/BACE model of $D$. melanogaster was characterised for the effect of CLU on phenotypic changes such as the crumpled wing phenotype, survival rates and alterations in cholesterol metabolism. It is essential that further characterisation of the APP/BACE fly is conducted due to the uncertainty of the BACE gene being incorporated into the CNS of the fly. BACE and gamma secretase levels should be quantified by immunoblotting to confirm sufficient expression of both genes within the fly. Utilisation of a gamma secretase inhibitor such as L-685,458 should be implemented in order to measure $\beta C T F$ in the fly through western blotting as well as assay has already been optimised with SWAPP SH-SY5Y cells. In order to incorporate the inhibitor within the fly, L-685,458 needs to be integrated into fly feed. Once all of the above have been achieved, FL-APP protein can once again be assessed within APP/BACE/CLU flies as the assay has already been optimised for FL-APP detection within the fly. It would be of interest if CLU also changed the expression of activities of BACE and gamma secretase within APP/BACE flies. This could also be examined within SWAPP $/ \mathrm{CLU}^{+/+}$to complement findings in flies. 
In addition to the above, it is important to also determine the role of CLU within a model where homeostatic processes are normal (WT genotype model organism). From data gathered from this thesis (section 2.8 (figure 2.22) and section 2.10 (figure 2.26)), it can be hypothesised that clusterin acts in a detrimental manner, where overexpression of clusterin would register normal functionalities in e.g. free cholesterol, as negative stress sensors. Therefore CLU would decrease active cholesterol through two mechanisms. First binding of free cholesterol with CLU will prevent cholesterol from being involved in normal homeostatic processes. Secondly, the binding of CLU to cholesterol will not emit stress sensors as CLU lowers normal levels of oxidative stress required for normal autophagic processes. Therefore there is a reduction in the autophagy system which leads to the accumulation of cholesterol plaques within the brain. Based on current findings within this thesis, there is a lack of information in this regard to draw any positive conclusions; however beta and gamma secretase levels in future experiments should be examined in whether effects in activity do change upon induction of clusterin in an AD model. However if BACE was indeed not incorporated into APP/BACE flies, this means that clusterin would inhibit cleavage activity of beta secretase and not gamma secretase, as FL-APP levels weren't changed in the fly model, whereas they were changed within the swAPP SHSY5Y AD model.

$A \beta_{42}$ levels should then once again be reassessed in APP/BACE/CLU flies, by using the Invitrogen ELISA kit as well as comparing results to previous ELISA kits which have had successful detection of $A \beta_{42}$ using the APP/BACE fly model. Alongside the crumpled wing phenotype, Chakraborty et al also identified the development of melanotic lesions in APP/BACE flies (Figure 2.7A). Preliminary experiments in this thesis also observed melanotic lesions occurring on the abdomen and proboscis of APP/BACE flies. The presence of melanotic lesions is due to in immune response within the body of the fly. It would be of interest to examine whether CLU ameliorates this defect, as CLU has anti-inflammatory properties.

Finally, even though CLU levels are shown to be present through restriction digests during the creation of CLU viable plasmids transfection in SH-SY5Y cells, 
and phenotypic eye colour in flies, it would be beneficial to show CLU expression through other techniques through either a proteomic approach using mass spectrometry or immunoblotting approach using western blotting. Preliminary experiments for detection of CLU involved immunoblotting using WT-SHSY5Y and ELAV/CLU samples as a control for optimisation experiments using goat anticlusterin/ APOJ antibody (Everest Biotech, Cat\# EB06929). Transfer conditions of $1 \mathrm{hr} 40 \mathrm{~min}, 2.15 \mathrm{~h}$ at room temperature and overnight $(16 \mathrm{~h})$ at $4^{\circ} \mathrm{C}$ were trialled with negative results. $0.2 \mu \mathrm{M}$ and $0.4 \mu \mathrm{M}$ PVDF membrane was also trialled. However CLU wasn't able to be detected in either sample. Therefore future experiments would be to either further optimise the western blot protocol is required or antibody replacement is need. As the ELAV/CLU fly is positive for CLU integration within the CNS (section 2.3.1), at least this sample would be expected to contain high levels of CLU which western blotting would detect CLU band around $35-37 \mathrm{kDa}$. CLU protein control should also be included to have full confidence that the purchased antibody is still functional.

\subsubsection{Further Investigation into the Effect of CLU on Cholesterol Metabolism}

Cholesterol analysis within in vitro and in vivo models was carried out using MALDI-TOF mass spectrometry and filipin staining. MALDI-TOF mass spectrometry proved successful for WT, APP/BACE, APP/BACE/CLU and ELAV/CLU fly lines; however, repeating these analyses with lipid standards would be necessary to insure cholesterol is indeed the lipid being measured. The MALDI-TOF was not useful for WT SH-SY5Y, sWAPP SH-SY5Y, and CLU expressing human cells given the low transfection efficiency and/or recovery of CLU-positive cells during FACS analysis. If sufficient levels of transfected cells was obtained with high purity of at least $80 \%$ (as FACS machine is capable of achieving), then cholesterol and other lipid levels could be considered as significant, as variability in readings produced by the MALDI-TOF machine would not be considered a big issue and will be statistically significant. Expected readings from $\mathrm{SH}-\mathrm{SY} 5 \mathrm{Y}$ cell lines would ideally reflect observations made within flies, as swAPP/CLU ${ }^{+/+}$levels should be high in comparison to SWAPP/CLU ${ }^{-/}$cells. If cell data does reflect fly 
cholesterol levels, then WT/CLU cells will have an increase of cholesterol in comparison to WT alone.

During Filipin staining on APP/BACE fly brains, a subset of staining was localised to the junction between the dorsal and medial lobes of the mushroom body. The mushroom body is responsible for learning and memory within the fly. This structure is important as it reflects anatomy involved in the limbic system in humans (including the hippocampus) which is affected within AD patients. To confirm findings, APP/BACE and APP/BACE/CLU brains will need to be stained for different regions of the brain alongside with Filipin staining to confirm where cholesterol aggregates lie. This will enable a brain map to be developed and will act as a reference as to which brain regions are specifically affected by cholesterol aggregation detrimental defects.

Preliminary experiments evaluating the structural integrity of the mushroom body (the learning and memory centre of the fly) were conducted using FASII staining within APP/BACE flies (Figure 4.1). The results suggested that there were defects within this structure, with the dorsal lobe primarily affected with either thinning or complete absence of the dorsal lobe (Figure 4.1C-F) in comparison to wild type (Figure 4.1A-B). FASII staining was also prominent in the central lobe where the axon bundles are located (Figure 4.1C, F). In addition, the FASIl staining may be useful for distinguishing the APP/BACE flies with and without crumpled wings. The crumpled wing phenotype does not completely segregate in the APP/BACE fly ${ }^{98}$. Further investigation of FASIl staining will establish a cellular phenotype to help understand the crumpled wing phenotype. Additional investigation of the mushroom body within APP/BACE/CLU and WT/CLU flies would be beneficial to determine whether CLU rescues this defect in APP/BACE. My demonstration that CLU rescues crumpled wing phenotype in the APP/BACE fly would predict that the FASII staining defect would also be rescued with CLU expression. These results would support a hypothesis that that CLU exhibits therapeutic effects by cholesterol efflux mechanisms.

Further investigation of cholesterol metabolism in human cells is required, but in order to do so, a few things must be optimized. First, in order to measure cholesterol using mass spectrometry, it is compulsory to optimize FACS 
analysis. FACS sorting for GFP/CLU positive cells should once again be attempted with the initial cell count within the sample to be processed, to be double of what was trialled out in this thesis, with only one run (compared to processing of the sample twice) to be conducted. This will intensify any changes in cholesterol within samples. Second, and alternatively to the first method, a higher yield of CLU expressing cells could be generated by producing a stable cell line that expresses CLU in WT and SWAPP SH-SY5Y cells. Third, standards for cholesterol and other lipids should be obtained and included in future MALDI-TOF analyses.

A

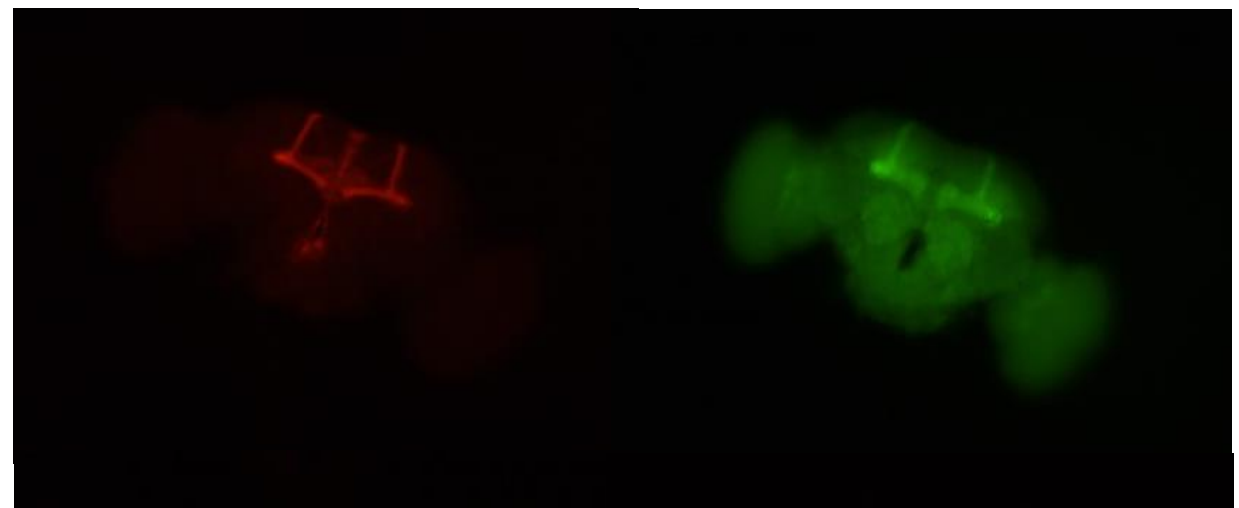

C

E

Figure 4.1: Immunohistochemistry FASII staining in AD flies reveals hindrance in structural integrity of mushroom bodies

(A)Wild type FASIl staining, (B) Wild type ELAV+/GFP, (C)APP695/BACE crumpled wing FASIl staining, d.APP695/BACE crumpled wing FASIl staining, e. APP695/BACE non-crumpled wing FASII staining, f. APP695/BACE non-crumpled wing FASII staining 
In $A D$ brains, it was found that in the prefrontal cortex (area of the brain responsible for decision making); there was an increase of diacylglycerol and sphingolipids. An increase of sphingomyelin, cholesterol esters, lysobisphophatidic acid and the ganglioside GM3 levels were also observed within the entorhinal cortex (area of the brain involved in memory processes) ${ }^{156}$. Therefore once MALDI-TOF experimental parameters have been optimised, cholesterol and other lipids should also be investigated in order to see if CLU has any unreported effects other than cholesterol e.g. sphingolipid and phospholipid content. Investigating other changes in lipid profiles may also elucidate cross-talk between different lipid pathways, and more broadly, help further understand the function of CLU in AD pathology.

\subsubsection{Further Characterization of the Effect of CLU on Autophagy}

Autophagy analysis was investigated within in vitro $A D$ model by the detection of LC3I-II and Lysotracker Red. Within cells, Lysotracker Red data visually proved promising for distinguishing sWAPP/CLU ${ }^{-/}$and SWAPP $/ \mathrm{CLU}^{+/+}$cells, however statistical analysis did not support a difference. Upon examination of datasets obtained from Image J quantification, 1-2 outlier cells out of the 100 quantified cells were present which would enlarge the standard error bars which would affect the true statistical result. In order to address this issue, the sample size of each cell genotype should be increased. It is also essential to complete the dataset of Lysotracker Red images of cells treated with bafilomycin to provide a bigger window to study autophagy and perhaps amplify the effects of CLU on autophagy.

Unfortunately, I was not able to characterise autophagy in the fly. The LC3 western blot assay that was used in cells does not work in flies if a mammalian LC3 antibody is being used, although the mechanisms behind this are unknown ${ }^{157}$. However western blotting for LC3 does work if a LC3 human protein is expressed within flies (GFP-LC3) or if a specific antibody is used for Atg8 $8^{157}$. । did attempt to quantify the levels of Lysotracker Red with respect to DAPI, an assay that is established to investigate autophagy in flies. However, my experiments proved unsuccessful. Suspicions lie in that either brains were stored 
long term in methanol for a long period of time which affected the uptake of the stain in the brain, or concentrations of staining were not optimal. Previous studies have successfully used Lysotracker Green in detection of acidified organelles within fly brains. Therefore in the future, these experiments can be tried again with further optimisation.

Ideally, fluorescent microscopy of filipin and Lysotracker staining would be utilized in the same sample. This technique requires further optimization as my preliminary experiments identified that a nitric acid wash on glass coverslips don't efficiently adhere transfected cells after fixation with paraformaldehyde. Poly-L-lysine adhesive coating was therefore utilized, however this distorts cell morphology, therefore it is questionable whether these results are reliable.

Trials of small amounts of laminin coated on top of polylysine adhesive coating was therefore used and shows promise in that it resolved adherence issues. Therefore, the methodology has been refined, but not yet completely optimised, to simultaneously investigate the effect of CLU on cholesterol and autophagy mechanisms. Alternatively, an indirect approach of visualising crosstalk between autophagy and cholesterol would be to use bafilomycin to inhibit the vATPase $\mathrm{H}^{+}$pump that is require. However, this method would be sensitive to the amount of bafilomycin given that bafilomycin can induce accumulation of cholesterol as detected with filipin. This method will only work using an amount of bafilomycin that disrupts autophagic flux without disrupting cholesterol transport in the autophagy system.

\subsubsection{Additional Mechanisms to Pursue}

An interesting component to test in $A D$ in vivo experiments would be to study how behaviour is influenced by CLU overexpression within APP/BACE flies. This can be achieved through courtship behaviour or assays using the T-maze. These assays would enhance the hypothesis of CLU inducing protective mechanisms to rescue symptoms of memory loss, or more broadly, directly test if CLU affects the onset and progression of neurodegeneration.

Previous studies which have investigated the role of CLU in flies have used stress related experiments to measure responses to ROS levels and oxidative 
stress, a phenomenon that is also dysregulated in AD. These experiments however have not been utilised in any fly model of AD. It would be interesting to investigate oxidative stress in the APP/BACE fly as well as APP/BACE/CLU fly to investigate a role for CLU in oxidative stress. 


\section{REFERENCES}

1 Nixon, R. A. The role of autophagy in neurodegenerative disease. Nature medicine 19, 983-997, doi:10.1038/nm.3232 (2013).

2 Ferri, C. P. et al. Global prevalence of dementia: a Delphi consensus study. Lancet 366, 2112-2117, doi:10.1016/s0140-6736(05)67889-0 (2005).

3 Maulik, M., Westaway, D., Jhamandas, J. H. \& Kar, S. Role of cholesterol in APP metabolism and its significance in Alzheimer's disease pathogenesis. Mol Neurobiol 47, 37-63, doi:10.1007/s12035-012-8337-y (2013).

4 Kim, J. et al. Overexpression of low-density lipoprotein receptor in the brain markedly inhibits amyloid deposition and increases extracellular A beta clearance. Neuron 64, 632-644, doi:10.1016/j.neuron.2009.11.013 (2009).

5 Marks, N. \& Berg, M. J. BACE and gamma-secretase characterization and their sorting as therapeutic targets to reduce amyloidogenesis.

Neurochemical research 35, 181-210, doi:10.1007/s11064-009-0054-1 (2010).

6 Tromp, D., Dufour, A., Lithfous, S., Pebayle, T. \& Despres, O. Episodic memory in normal aging and Alzheimer disease: Insights from imaging and behavioral studies. Ageing research reviews, doi:10.1016/j.arr.2015.08.006 (2015).

7 van Es, M. A. \& van den Berg, L. H. Alzheimer's disease beyond APOE. Nat Genet 41, 1047-1048, doi:10.1038/ng1009-1047 (2009).

8 Bhattacharyya, R. \& Kovacs, D. M. ACAT inhibition and amyloid beta reduction. Biochimica et biophysica acta 1801, 960-965, doi:10.1016/j.bbalip.2010.04.003 (2010).

9 Mori, T. et al. Cholesterol accumulates in senile plaques of Alzheimer disease patients and in transgenic $\operatorname{APP}(\mathrm{SW})$ mice. Journal of neuropathology and experimental neurology 60, 778-785 (2001).

10 Sparks, D. L. et al. Induction of Alzheimer-like beta-amyloid immunoreactivity in the brains of rabbits with dietary cholesterol. Experimental neurology 126, 88-94, doi:10.1006/exnr.1994.1044 (1994). 
11 Fassbender, K. et al. Simvastatin strongly reduces levels of Alzheimer's disease beta -amyloid peptides Abeta 42 and Abeta 40 in vitro and in vivo. Proceedings of the National Academy of Sciences of the United States of America 98, 5856-5861, doi:10.1073/pnas.081620098 (2001).

12 Cibickova, L. et al. The influence of simvastatin, atorvastatin and highcholesterol diet on acetylcholinesterase activity, amyloid beta and cholesterol synthesis in rat brain. Steroids 74, 13-19, doi:10.1016/j.steroids.2008.08.007 (2009).

13 Refolo, L. M. et al. A cholesterol-lowering drug reduces beta-amyloid pathology in a transgenic mouse model of Alzheimer's disease. Neurobiology of disease 8, 890-899, doi:10.1006/nbdi.2001.0422 (2001).

14 Petanceska, S. S. et al. Statin therapy for Alzheimer's disease: will it work? Journal of molecular neuroscience : MN 19, 155-161, doi:10.1007/s12031-002-0026-2 (2002).

15 Park, I. H. et al. Lovastatin enhances Abeta production and senile plaque deposition in female Tg2576 mice. Neurobiology of aging 24, 637-643 (2003).

16 Kim, J., Basak, J. M. \& Holtzman, D. M. The Role of Apolipoprotein E in Alzheimer's Disease. Neuron 63, 287-303, doi:10.1016/j.neuron.2009.06.026 (2009).

17 Zhang, J. \& Liu, Q. Cholesterol metabolism and homeostasis in the brain. Protein \& Cell 6, 254-264, doi:10.1007/s13238-014-0131-3 (2015).

18 Tall, A. R. Cholesterol efflux pathways and other potential mechanisms involved in the athero-protective effect of high density lipoproteins. Journal of internal medicine 263, 256-273, doi:10.1111/j.13652796.2007.01898.x (2008).

19 Hirsch-Reinshagen, V. et al. The absence of ABCA1 decreases soluble ApoE levels but does not diminish amyloid deposition in two murine models of Alzheimer disease. The Journal of biological chemistry 280, 43243-43256, doi:10.1074/jbc.M508781200 (2005).

20 Puglielli, L. et al. Acyl-coenzyme A: cholesterol acyltransferase modulates the generation of the amyloid beta-peptide. Nature cell biology 3, 905-912, doi:10.1038/ncb1001-905 (2001). 
21 Hutter-Paier, B. et al. The ACAT inhibitor CP-113,818 markedly reduces amyloid pathology in a mouse model of Alzheimer's disease. Neuron 44, 227-238, doi:10.1016/j.neuron.2004.08.043 (2004).

22 Grimm, M. O., Grimm, H. S. \& Hartmann, T. Amyloid beta as a regulator of lipid homeostasis. Trends in molecular medicine 13, 337-344, doi:10.1016/j.molmed.2007.06.004 (2007).

23 Vetrivel, K. S. \& Thinakaran, G. Membrane rafts in Alzheimer's disease beta-amyloid production. Biochimica et biophysica acta 1801, 860-867, doi:10.1016/j.bbalip.2010.03.007 (2010).

24 Kuo, Y. M. et al. Water-soluble Abeta (N-40, N-42) oligomers in normal and Alzheimer disease brains. The Journal of biological chemistry 271, 4077-4081 (1996).

25 Petanceska, S. S. et al. Changes in apolipoprotein E expression in response to dietary and pharmacological modulation of cholesterol. Journal of molecular neuroscience : MN 20, 395-406, doi:10.1385/jmn:20:3:395 (2003).

26 Hui, L., Chen, X. \& Geiger, J. D. Endolysosome involvement in LDL cholesterol-induced Alzheimer's disease-like pathology in primary cultured neurons. Life sciences 91, 1159-1168, doi:10.1016/j.lfs.2012.04.039 (2012).

27 Nixon, R. A. \& Yang, D.-S. Autophagy Failure in Alzheimer's Disease Locating the Primary Defect. Neurobiology of disease 43, 38-45, doi:10.1016/j.nbd.2011.01.021 (2011).

28 Jia, Y. et al. Ursolic acid improves lipid and glucose metabolism in highfat-fed C57BL/6J mice by activating peroxisome proliferator-activated receptor alpha and hepatic autophagy. Molecular nutrition \& food research 59, 344-354, doi:10.1002/mnfr.201400399 (2015).

29 Huang, B. et al. Simvastatin enhances oxidizedlow density lipoproteininduced macrophage autophagy and attenuates lipid aggregation. Molecular medicine reports 11, 1093-1098, doi:10.3892/mmr.2014.2790 (2015).

30 Wang, J. et al. Liraglutide protects pancreatic beta-cells against free fatty acids in vitro and affects glucolipid metabolism in apolipoprotein E/ mice 
by activating autophagy. Molecular medicine reports 12, 4210-4218, doi:10.3892/mmr.2015.3944 (2015).

31 Shibuya, Y. et al. Acyl-coenzyme A:cholesterol acyltransferase 1 blockage enhances autophagy in the neurons of triple transgenic Alzheimer's disease mouse and reduces human P301L-tau content at the presymptomatic stage. Neurobiology of aging 36, 2248-2259, doi:10.1016/j.neurobiolaging.2015.04.002 (2015).

32 Yang, D. S. et al. Defective macroautophagic turnover of brain lipids in the TgCRND8 Alzheimer mouse model: prevention by correcting lysosomal proteolytic deficits. Brain : a journal of neurology 137, 33003318, doi:10.1093/brain/awu278 (2014).

33 de Silva, H. V. et al. A 70-kDa apolipoprotein designated ApoJ is a marker for subclasses of human plasma high density lipoproteins. The Journal of biological chemistry 265, 13240-13247 (1990).

34 Kirszbaum, L. et al. Molecular cloning and characterization of the novel, human complement-associated protein, SP-40,40: a link between the complement and reproductive systems. The EMBO journal 8, 711-718 (1989).

35 Jenne, D. E. \& Tschopp, J. Molecular structure and functional characterization of a human complement cytolysis inhibitor found in blood and seminal plasma: identity to sulfated glycoprotein 2, a constituent of rat testis fluid. Proceedings of the National Academy of Sciences of the United States of America 86, 7123-7127 (1989).

36 Miyake, H., Nelson, C., Rennie, P. S. \& Gleave, M. E. Testosteronerepressed prostate message- 2 is an antiapoptotic gene involved in progression to androgen independence in prostate cancer. Cancer research 60, 170-176 (2000).

37 Yang, C. R. et al. Nuclear clusterin/XIP8, an x-ray-induced Ku70-binding protein that signals cell death. Proceedings of the National Academy of Sciences of the United States of America 97, 5907-5912 (2000).

38 Sensibar, J. A. et al. Prevention of cell death induced by tumor necrosis factor alpha in LNCaP cells by overexpression of sulfated glycoprotein-2 (clusterin). Cancer research 55, 2431-2437 (1995). 
39 Jenne, D. E. \& Tschopp, J. Clusterin: the intriguing guises of a widely expressed glycoprotein. Trends in biochemical sciences 17, 154-159 (1992).

40 Fink, T. M. et al. Human clusterin (CLI) maps to 8p21 in proximity to the lipoprotein lipase (LPL) gene. Genomics 16, 526-528, doi:10.1006/geno.1993.1222 (1993).

41 Tsuruta, J. K., Wong, K., Fritz, I. B. \& Griswold, M. D. Structural analysis of sulphated glycoprotein 2 from amino acid sequence. Relationship to clusterin and serum protein 40,40. The Biochemical journal 268, 571-578 (1990).

42 Herault, Y., Chatelain, G., Brun, G. \& Michel, D. V-src-inducedtranscription of the avian clusterin gene. Nucleic acids research $\mathbf{2 0}, 6377$ 6383 (1992).

43 Michel, D., Moyse, E., Trembleau, A., Jourdan, F. \& Brun, G. Clusterin/ApoJ expression is associated with neuronal apoptosis in the olfactory mucosa of the adult mouse. Journal of cell science 110 ( Pt 14), 1635-1645 (1997).

44 Wong, P. et al. Genomic organization and expression of the rat TRPM-2 (clusterin) gene, a gene implicated in apoptosis. The Journal of biological chemistry 268, 5021-5031 (1993).

45 Wong, P. et al. Increased TRPM-2/clusterin mRNA levels during the time of retinal degeneration in mouse models of retinitis pigmentosa.

Biochemistry and cell biology = Biochimie et biologie cellulaire 72, 439446 (1994).

46 Rosenberg, M. E. \& Silkensen, J. Clusterin: physiologic and pathophysiologic considerations. The international journal of biochemistry \& cell biology 27, 633-645 (1995).

47 Jordan-Starck, T. C. et al. Mouse apolipoprotein J: characterization of a gene implicated in atherosclerosis. Journal of lipid research 35, 194-210 (1994).

48 Kelso, G. J. et al. Apolipoprotein J is associated with paraoxonase in human plasma. Biochemistry 33, 832-839 (1994).

49 Aronow, B. J., Lund, S. D., Brown, T. L., Harmony, J. A. \& Witte, D. P. Apolipoprotein $\mathrm{J}$ expression at fluid-tissue interfaces: potential role in 
barrier cytoprotection. Proceedings of the National Academy of Sciences of the United States of America 90, 725-729 (1993).

50 Bailey, R. W., Dunker, A. K., Brown, C. J., Garner, E. C. \& Griswold, M. D. Clusterin, a binding protein with a molten globule-like region.

Biochemistry 40, 11828-11840 (2001).

51 Collard, M. W. \& Griswold, M. D. Biosynthesis and molecular cloning of sulfated glycoprotein 2 secreted by rat Sertoli cells. Biochemistry 26, 3297-3303 (1987).

52 Cyr, D. G. \& Robaire, B. Regulation of sulfated glycoprotein-2 (clusterin) messenger ribonucleic acid in the rat epididymis. Endocrinology 130, 2160-2166, doi:10.1210/endo.130.4.1547732 (1992).

53 Wong, P. et al. Molecular characterization of human TRPM-2/clusterin, a gene associated with sperm maturation, apoptosis and neurodegeneration. European journal of biochemistry / FEBS 221, 917-925 (1994).

54 Choi, N. H., Mazda, T. \& Tomita, M. A serum protein SP40,40 modulates the formation of membrane attack complex of complement on erythrocytes. Molecular immunology 26, 835-840 (1989).

55 Dai, D.-F., Chiao, Y. A., Marcinek, D. J., Szeto, H. H. \& Rabinovitch, P. S. Mitochondrial oxidative stress in aging and healthspan. Longevity \& Healthspan 3, 6-6, doi:10.1186/2046-2395-3-6 (2014).

56 Benz, C. C. \& Yau, C. Ageing, oxidative stress and cancer: paradigms in parallax. Nature reviews. Cancer 8, 875-879, doi:10.1038/nrc2522 (2008).

57 Criswell, T., Klokov, D., Beman, M., Lavik, J. P. \& Boothman, D. A. Repression of IR-inducible clusterin expression by the p53 tumor suppressor protein. Cancer biology \& therapy 2, 372-380 (2003).

58 Klokov, D. et al. IR-inducible clusterin gene expression: a protein with potential roles in ionizing radiation-induced adaptive responses, genomic instability, and bystander effects. Mutation research 568, 97-110, doi:10.1016/j.mrfmmm.2004.06.049 (2004).

59 Danik, M., Chabot, J. G., Hassan-Gonzalez, D., Suh, M. \& Quirion, R. Localization of sulfated glycoprotein-2/clusterin mRNA in the rat brain by in situ hybridization. The Journal of comparative neurology 334, 209-227, doi:10.1002/cne.903340205 (1993). 
60 Garden, G. A., Bothwell, M. \& Rubel, E. W. Lack of correspondence between mRNA expression for a putative cell death molecule (SGP-2) and neuronal cell death in the central nervous system. Journal of neurobiology 22, 590-604, doi:10.1002/neu.480220605 (1991).

61 O'Bryan, M. K., Cheema, S. S., Bartlett, P. F., Murphy, B. F. \& Pearse, M. J. Clusterin levels increase during neuronal development. Journal of neurobiology 24, 421-432, doi:10.1002/neu.480240402 (1993).

62 Pasinetti, G. M., Johnson, S. A., Oda, T., Rozovsky, I. \& Finch, C. E. Clusterin (SGP-2): a multifunctional glycoprotein with regional expression in astrocytes and neurons of the adult rat brain. The Journal of comparative neurology 339, 387-400, doi:10.1002/cne.903390307 (1994).

63 Zlokovic, B. V. et al. Glycoprotein 330/megalin: probable role in receptormediated transport of apolipoprotein $\mathrm{J}$ alone and in a complex with Alzheimer disease amyloid beta at the blood-brain and bloodcerebrospinal fluid barriers. Proceedings of the National Academy of Sciences of the United States of America 93, 4229-4234 (1996).

64 Oda, T. et al. Clusterin (apoJ) alters the aggregation of amyloid betapeptide (A beta 1-42) and forms slowly sedimenting A beta complexes that cause oxidative stress. Experimental neurology 136, 22-31 (1995).

65 Boggs, L. N. et al. Clusterin (Apo J) protects against in vitro amyloid-beta (1-40) neurotoxicity. J Neurochem 67, 1324-1327 (1996).

66 Matsubara, E., Soto, C., Governale, S., Frangione, B. \& Ghiso, J. Apolipoprotein J and Alzheimer's amyloid beta solubility. Biochemical Journal 316, 671-679 (1996).

67 May, P. C. \& Finch, C. E. Sulfated glycoprotein 2: new relationships of this multifunctional protein to neurodegeneration. Trends in neurosciences 15, 391-396 (1992).

68 DeMattos, R. B. et al. Clusterin promotes amyloid plaque formation and is critical for neuritic toxicity in a mouse model of Alzheimer's disease. Proceedings of the National Academy of Sciences of the United States of America 99, 10843-10848, doi:10.1073/pnas.162228299 (2002).

69 Bettens, K. et al. Both common variations and rare non-synonymous substitutions and small insertion/deletions in CLU are associated with 
increased Alzheimer risk. Mol Neurodegener 7, 3, doi:10.1186/17501326-7-3 (2012).

70 Holtzman, D. M. In vivo effects of ApoE and clusterin on amyloid-beta metabolism and neuropathology. Journal of molecular neuroscience : MN 23, 247-254, doi:10.1385/jmn:23:3:247 (2004).

71 Bell, R. D. et al. Transport pathways for clearance of human Alzheimer's amyloid beta-peptide and apolipoproteins $\mathrm{E}$ and $\mathrm{J}$ in the mouse central nervous system. Journal of cerebral blood flow and metabolism : official journal of the International Society of Cerebral Blood Flow and Metabolism 27, 909-918, doi:10.1038/sj.jcbfm.9600419 (2007).

72 Narayan, P. et al. The extracellular chaperone clusterin sequesters oligomeric forms of the amyloid-beta(1-40) peptide. Nature structural \& molecular biology 19, 79-83, doi:10.1038/nsmb.2191 (2012).

73 Howlett, D. R., Hortobagyi, T. \& Francis, P. T. Clusterin associates specifically with Abeta40 in Alzheimer's disease brain tissue. Brain pathology (Zurich, Switzerland) 23, 623-632, doi:10.1111/bpa.12057 (2013).

74 de Silva, H. V., Harmony, J. A., Stuart, W. D., Gil, C. M. \& Robbins, J. Apolipoprotein J: structure and tissue distribution. Biochemistry 29, 53805389 (1990).

75 James, R. W. et al. Characterization of a human high density lipoproteinassociated protein, NA1/NA2. Identity with SP-40,40, an inhibitor of complement-mediated cytolysis. Arteriosclerosis and thrombosis : a journal of vascular biology / American Heart Association 11, 645-652 (1991).

76 Eisenberg, S. High density lipoprotein metabolism. Journal of lipid research 25, 1017-1058 (1984).

77 Seo, H. Y. et al. Clusterin decreases hepatic SREBP-1c expression and lipid accumulation. Endocrinology 154, 1722-1730, doi:10.1210/en.20122009 (2013).

78 Gelissen, I. C. et al. Apolipoprotein J (clusterin) induces cholesterol export from macrophage-foam cells: a potential anti-atherogenic function?

Biochemical Journal 331, 231-237 (1998). 
79 Zhang, F. et al. Clusterin facilitates stress-induced lipidation of LC3 and autophagosome biogenesis to enhance cancer cell survival. Nature communications 5, 5775, doi:10.1038/ncomms6775 (2014).

80 Biedler, J. L., Helson, L. \& Spengler, B. A. Morphology and growth, tumorigenicity, and cytogenetics of human neuroblastoma cells in continuous culture. Cancer research 33, 2643-2652 (1973).

81 Korecka, J. A. et al. Phenotypic characterization of retinoic acid differentiated SH-SY5Y cells by transcriptional profiling. PloS one $\mathbf{8}$, e63862, doi:10.1371/journal.pone.0063862 (2013).

82 Presgraves, S. P., Ahmed, T., Borwege, S. \& Joyce, J. N. Terminally differentiated SH-SY5Y cells provide a model system for studying neuroprotective effects of dopamine agonists. Neurotoxicity research $\mathbf{5}$, 579-598 (2004).

83 Jamsa, A., Hasslund, K., Cowburn, R. F., Backstrom, A. \& Vasange, M. The retinoic acid and brain-derived neurotrophic factor differentiated $\mathrm{SH}$ SY5Y cell line as a model for Alzheimer's disease-like tau phosphorylation. Biochemical and biophysical research communications 319, 993-1000, doi:10.1016/j.bbrc.2004.05.075 (2004).

84 Encinas, M. et al. Sequential treatment of SH-SY5Y cells with retinoic acid and brain-derived neurotrophic factor gives rise to fully differentiated, neurotrophic factor-dependent, human neuron-like cells. J Neurochem $\mathbf{7 5}$, 991-1003 (2000).

85 Sanfeliu, C., Cristofol, R., Toran, N., Rodriguez-Farre, E. \& Kim, S. U. Use of human central nervous system cell cultures in neurotoxicity testing. Toxicology in vitro : an international journal published in association with BIBRA 13, 753-759 (1999).

86 Beyreuther, K. et al. Regulation and expression of the Alzheimer's beta/A4 amyloid protein precursor in health, disease, and Down's syndrome. Annals of the New York Academy of Sciences 695, 91-102 (1993).

87 Belyaev, N. D. et al. The transcriptionally active amyloid precursor protein (APP) intracellular domain is preferentially produced from the 695 isoform of APP in a \{beta\}-secretase-dependent pathway. The Journal of biological chemistry 285, 41443-41454, doi:10.1074/jbc.M110.141390 (2010). 

disease by beta-secretase cleavage within the secretory pathway. Nature medicine 1, 1291-1296 (1995).

89 Citron, M. et al. Mutation of the beta-amyloid precursor protein in familial Alzheimer's disease increases beta-protein production. Nature 360, 672674, doi:10.1038/360672a0 (1992).

90 Mullan, M. et al. A pathogenic mutation for probable Alzheimer's disease in the APP gene at the N-terminus of beta-amyloid. Nat Genet 1, 345-347, doi:10.1038/ng0892-345 (1992).

91 Adams, M. D. et al. The genome sequence of Drosophila melanogaster. Science (New York, N.Y.) 287, 2185-2195 (2000).

92 Nichols, C. D. Drosophila melanogaster neurobiology, neuropharmacology, and how the fly can inform central nervous system drug discovery. Pharmacology \& therapeutics 112, 677-700, doi:10.1016/j.pharmthera.2006.05.012 (2006).

93 McGuire, S. E., Deshazer, M. \& Davis, R. L. Thirty years of olfactory learning and memory research in Drosophila melanogaster. Progress in neurobiology 76, 328-347, doi:10.1016/j.pneurobio.2005.09.003 (2005).

94 Greenspan, R. J. \& Dierick, H. A. ‘Am not I a fly like thee?' From genes in fruit flies to behavior in humans. Human Molecular Genetics 13, R267R273, doi:10.1093/hmg/ddh248 (2004).

95 Fortini, M. E., Skupski, M. P., Boguski, M. S. \& Hariharan, I. K. A survey of human disease gene counterparts in the Drosophila genome. The Journal of cell biology 150, F23-30 (2000).

96 Periz, G. \& Fortini, M. E. Functional reconstitution of gamma-secretase through coordinated expression of presenilin, nicastrin, Aph-1, and Pen-2. Journal of neuroscience research 77, 309-322, doi:10.1002/jnr.20203 (2004).

97 Luo, L., Tully, T. \& White, K. Human amyloid precursor protein ameliorates behavioral deficit of flies deleted for Appl gene. Neuron $\mathbf{9}$, 595-605 (1992).

98 Chakraborty, R. et al. Characterization of a Drosophila Alzheimer's Disease Model: Pharmacological Rescue of Cognitive Defects. PloS one 6, e20799, doi:10.1371/journal.pone.0020799 (2011). 
99 Phelps, C. B. \& Brand, A. H. Ectopic gene expression in Drosophila using GAL4 system. Methods (San Diego, Calif.) 14, 367-379, doi:10.1006/meth.1998.0592 (1998).

100 Lee, Y. N., Shim, Y. J., Kang, B. H., Park, J. J. \& Min, B. H. Overexpression of human clusterin increases stress resistance and extends lifespan in Drosophila melanogaster. Biochemical and biophysical research communications 420, 851-856, doi:10.1016/j.bbrc.2012.03.087 (2012).

101 Zhou, Y. et al. Intracellular clusterin interacts with brain isoforms of the bridging integrator 1 and with the microtubule-associated protein Tau in Alzheimer's disease. PloS one 9, e103187, doi:10.1371/journal.pone.0103187 (2014).

102 Spradling, A. C. \& Rubin, G. M. Transposition of cloned P elements into Drosophila germ line chromosomes. Science (New York, N.Y.) 218, 341347 (1982).

103 Bligh, E. G. \& Dyer, W. J. A RAPID METHOD OF TOTAL LIPID EXTRACTION AND PURIFICATION. Canadian Journal of Biochemistry and Physiology 37, 911-917, doi:10.1139/o59-099 (1959).

104 Guan, X. L. et al. Biochemical membrane lipidomics during Drosophila development. Developmental cell 24, 98-111, doi:10.1016/j.devcel.2012.11.012 (2013).

105 Cataldo, A. M. et al. Down syndrome fibroblast model of Alzheimerrelated endosome pathology: accelerated endocytosis promotes late endocytic defects. The American journal of pathology 173, 370-384, doi:10.2353/ajpath.2008.071053 (2008).

106 Gotz, J. \& Ittner, L. M. Animal models of Alzheimer's disease and frontotemporal dementia. Nature reviews. Neuroscience 9, 532-544, doi:http://www.nature.com/nrn/journal/v9/n7/suppinfo/nrn2420_S1.html (2008).

107 Van Dam, D. \& De Deyn, P. P. Animal models in the drug discovery pipeline for Alzheimer's disease. British Journal of Pharmacology 164, 1285-1300, doi:10.1111/j.1476-5381.2011.01299.x (2011). 
108 Haass, C. et al. The Swedish mutation causes early-onset Alzheimer's disease by [beta]-secretase cleavage within the secretory pathway. Nature medicine 1, 1291-1296 (1995).

109 Di, X. et al. L-theanine protects the APP (Swedish mutation) transgenic SH-SY5Y cell against glutamate-induced excitotoxicity via inhibition of the NMDA receptor pathway. Neuroscience 168, 778-786, doi:10.1016/j.neuroscience.2010.04.019 (2010).

110 Jones, N. Alzheimer disease: plasma clusterin predicts degree of pathogenesis in AD. Nature reviews. Neurology 6, 469, doi:10.1038/nrneurol.2010.122 (2010).

111 Thambisetty, M. et al. Association of plasma clusterin concentration with severity, pathology, and progression in Alzheimer disease. Archives of general psychiatry 67, 739-748, doi:10.1001/archgenpsychiatry.2010.78 (2010).

112 Rostagno, A. \& Ghiso, J. ISOLATION AND BIOCHEMICAL CHARACTERIZATION OF AMYLOID PLAQUES AND PAIRED HELICAL FILAMENTS. Current protocols in cell biology / editorial board, Juan S. Bonifacino ... [et al.] CHAPTER, Unit-3.3333, doi:10.1002/0471143030.cb0333s44 (2009).

113 Izco, M., Pesini, P., Perez-Grijalba, V., Fandos, N. \& Sarasa, M. Optimized protocol for amyloid-beta extraction from the brain. Journal of Alzheimer's disease : JAD 34, 835-839, doi:10.3233/jad-121798 (2013).

114 Marcora, M. S. et al. Amyloid peptides ABri and ADan show differential neurotoxicity in transgenic Drosophila models of familial British and Danish dementia. Mol Neurodegener 9, 5, doi:10.1186/1750-1326-9-5 (2014).

115 DeMattos, R. B. et al. ApoE and clusterin cooperatively suppress Abeta levels and deposition: evidence that ApoE regulates extracellular Abeta metabolism in vivo. Neuron 41, 193-202 (2004).

116 Parisi, M., Li, R. \& Oliver, B. Lipid profiles of female and male Drosophila. BMC research notes 4, 198, doi:10.1186/1756-0500-4-198 (2011).

117 Suarez, I., Bodega, G. \& Fernandez, B. Glutamine synthetase in brain: effect of ammonia. Neurochemistry international 41, 123-142 (2002). 
118 Sofroniew, M. V. \& Vinters, H. V. Astrocytes: biology and pathology. Acta Neuropathologica 119, 7-35, doi:10.1007/s00401-009-0619-8 (2010).

119 Olabarria, M., Noristani, H. N., Verkhratsky, A. \& Rodriguez, J. J. Agedependent decrease in glutamine synthetase expression in the hippocampal astroglia of the triple transgenic Alzheimer's disease mouse model: mechanism for deficient glutamatergic transmission? Mol Neurodegener $\mathbf{6}$, 55, doi:10.1186/1750-1326-6-55 (2011).

120 Robinson, S. R. Neuronal expression of glutamine synthetase in Alzheimer's disease indicates a profound impairment of metabolic interactions with astrocytes. Neurochemistry international 36, 471-482 (2000).

121 Castro Mdel, R. et al. Aging increases mitochondrial DNA damage and oxidative stress in liver of rhesus monkeys. Experimental gerontology 47, 29-37, doi:10.1016/j.exger.2011.10.002 (2012).

122 Kwon, H. S. et al. Clusterin expression level correlates with increased oxidative stress in asthmatics. Annals of allergy, asthma \& immunology : official publication of the American College of Allergy, Asthma, \& Immunology 112, 217-221, doi:10.1016/j.anai.2013.12.012 (2014).

123 Carver, J. A., Rekas, A., Thorn, D. C. \& Wilson, M. R. Small heat-shock proteins and clusterin: intra- and extracellular molecular chaperones with a common mechanism of action and function? IUBMB life 55, 661-668, doi:10.1080/15216540310001640498 (2003).

124 Wyatt, A. R. et al. Clusterin facilitates in vivo clearance of extracellular misfolded proteins. Cellular and molecular life sciences : CMLS 68, 39193931, doi:10.1007/s00018-011-0684-8)

10.1007/s00018-011-0684-8 (2011).

125 Poon, S., Easterbrook-Smith, S. B., Rybchyn, M. S., Carver, J. A. \& Wilson, M. R. Clusterin is an ATP-independent chaperone with very broad substrate specificity that stabilizes stressed proteins in a folding-competent state. Biochemistry 39, 15953-15960 (2000).

126 Lamming, D. W., Ye, L., Sabatini, D. M. \& Baur, J. A. Rapalogs and mTOR inhibitors as anti-aging therapeutics. The Journal of clinical investigation 123, 980-989, doi:10.1172/jci64099 (2013). 
127 Hurez, V. et al. Chronic mTOR inhibition in mice with rapamycin alters T, $\mathrm{B}$, myeloid, and innate lymphoid cells and gut flora and prolongs life of immune-deficient mice. Aging cell, doi:10.1111/acel.12380 (2015).

128 Kapahi, P. et al. Regulation of lifespan in Drosophila by modulation of genes in the TOR signaling pathway. Current biology : $C B$ 14, 885-890, doi:10.1016/j.cub.2004.03.059 (2004).

129 Vellai, T. et al. Genetics: influence of TOR kinase on lifespan in C. elegans. Nature 426, 620, doi:10.1038/426620a (2003).

130 Wood, K. C. \& Sabatini, D. M. Growth signaling at the nexus of stem cell life and death. Cell stem cell 5, 232-234, doi:10.1016/j.stem.2009.08.008 (2009).

131 Mueller, M. A., Beutner, F., Teupser, D., Ceglarek, U. \& Thiery, J. Prevention of atherosclerosis by the mTOR inhibitor everolimus in LDLR/- mice despite severe hypercholesterolemia. Atherosclerosis 198, 39-48, doi:10.1016/j.atherosclerosis.2007.09.019 (2008).

132 Pakala, R., Stabile, E., Jang, G. J., Clavijo, L. \& Waksman, R. Rapamycin attenuates atherosclerotic plaque progression in apolipoprotein $\mathrm{E}$ knockout mice: inhibitory effect on monocyte chemotaxis. Journal of cardiovascular pharmacology 46, 481-486 (2005).

133 Spilman, P. et al. Inhibition of mTOR by rapamycin abolishes cognitive deficits and reduces amyloid-beta levels in a mouse model of Alzheimer's disease. PloS one 5, e9979, doi:10.1371/journal.pone.0009979 (2010).

134 Caccamo, A., Majumder, S., Richardson, A., Strong, R. \& Oddo, S. Molecular interplay between mammalian target of rapamycin (mTOR), amyloid-beta, and Tau: effects on cognitive impairments. The Journal of biological chemistry 285, 13107-13120, doi:10.1074/jbc.M110.100420 (2010).

135 Majumder, S., Richardson, A., Strong, R. \& Oddo, S. Inducing autophagy by rapamycin before, but not after, the formation of plaques and tangles ameliorates cognitive deficits. PloS one 6, e25416, doi:10.1371/journal.pone.0025416 (2011).

136 Richardson, A., Galvan, V., Lin, A. L. \& Oddo, S. How longevity research can lead to therapies for Alzheimer's disease: The rapamycin story. 
Experimental gerontology 68, 51-58, doi:10.1016/j.exger.2014.12.002 (2015).

137 Chau, S., Herrmann, N., Ruthirakuhan, M. T., Chen, J. J. \& Lanctot, K. L. Latrepirdine for Alzheimer's disease. The Cochrane database of systematic reviews 4, Cd009524, doi:10.1002/14651858.CD009524.pub2 (2015).

138 Turner, R. S. et al. A randomized, double-blind, placebo-controlled trial of resveratrol for Alzheimer disease. Neurology, doi:10.1212/wnl.0000000000002035 (2015).

139 Alagiakrishnan, K., Sankaralingam, S., Ghosh, M., Mereu, L. \& Senior, P. Antidiabetic drugs and their potential role in treating mild cognitive impairment and Alzheimer's disease. Discovery medicine 16, 277-286 (2013).

140 Tanaka, N. K., Suzuki, E., Dye, L., Ejima, A. \& Stopfer, M. Dye Fills Reveal Additional Olfactory Tracts in the Protocerebrum of Wild-Type Drosophila. The Journal of comparative neurology 520, 4131-4140, doi:10.1002/cne.23149 (2012).

141 Ito, K. et al. A systematic nomenclature for the insect brain. Neuron 81, 755-765, doi:10.1016/j.neuron.2013.12.017 (2014).

142 Tanaka, N. K., Tanimoto, H. \& Ito, K. Neuronal assemblies of the Drosophila mushroom body. The Journal of comparative neurology $\mathbf{5 0 8}$, 711-755, doi:10.1002/cne.21692 (2008).

143 Strutz, A. et al. Decoding odor quality and intensity in the Drosophila brain. eLife 3, e04147, doi:10.7554/eLife.04147 (2014).

144 Gupta, N. \& Stopfer, M. Functional analysis of a higher olfactory center, the lateral horn. The Journal of Neuroscience 32, 8138-8148, doi:10.1523/JNEUROSCI.1066-12.2012 (2012).

145 Yapici, N., Zimmer, M. \& Domingos, A. I. Cellular and molecular basis of decision-making. EMBO reports 15, 1023-1035, doi:10.15252/embr.201438993 (2014).

146 Wood, J. et al. Structure and function of the amygdaloid NPY system: NPY Y2 receptors regulate excitatory and inhibitory synaptic transmission in the centromedial amygdala. Brain structure \& function, doi:10.1007/s00429-015-1107-7 (2015). 
147 Greeve, I. et al. Age-dependent neurodegeneration and Alzheimer-amyloid plaque formation in transgenic Drosophila. The Journal of neuroscience : the official journal of the Society for Neuroscience 24, 3899-3906, doi:10.1523/jneurosci.0283-04.2004 (2004).

148 Wanamaker, B. L., Swiger, K. J., Blumenthal, R. S. \& Martin, S. S. Cholesterol, statins, and dementia: what the cardiologist should know. Clinical cardiology 38, 243-250, doi:10.1002/clc.22361 (2015).

149 Rosenson, R. S., Tangney, C. C. \& Casey, L. C. Inhibition of proinflammatory cytokine production by pravastatin. The Lancet 353, 983984, doi:10.1016/S0140-6736(98)05917-0.

150 Metais, C. et al. Simvastatin treatment preserves synaptic plasticity in AbetaPPswe/PS1dE9 mice. Journal of Alzheimer's disease : JAD 39, 315329, doi:10.3233/jad-130257 (2014).

151 Kurata, T. et al. Atorvastatin and pitavastatin reduce oxidative stress and improve IR/LDL-R signals in Alzheimer's disease. Neurological research 35, 193-205, doi:10.1179/1743132812y.0000000127 (2013).

152 Haag, M. D. M., Hofman, A., Koudstaal, P. J., Stricker, B. H. C. \& Breteler, M. M. B. Statins are associated with a reduced risk of Alzheimer disease regardless of lipophilicity. The Rotterdam Study. Journal of Neurology, Neurosurgery \& Psychiatry 80, 13-17, doi:10.1136/jnnp.2008.150433 (2009).

153 World_Health_Organisation. Dementia. Available from: http://www.who.int/mediacentre/factsheets/fs362/en/ (2015).

154 Groth, A. C., Fish, M., Nusse, R. \& Calos, M. P. Construction of transgenic Drosophila by using the site-specific integrase from phage phiC31. Genetics 166, 1775-1782 (2004).

155 Mizushima, N. \& Yoshimori, T. How to interpret LC3 immunoblotting. Autophagy 3, 542-545 (2007).

156 Chan, R. B. et al. Comparative Lipidomic Analysis of Mouse and Human Brain with Alzheimer Disease. Journal of Biological Chemistry 287, 2678-2688, doi:10.1074/jbc.M111.274142 (2012).

157 Klionsky, D. J. et al. Guidelines for the use and interpretation of assays for monitoring autophagy. Autophagy 8, 445-544 (2012). 


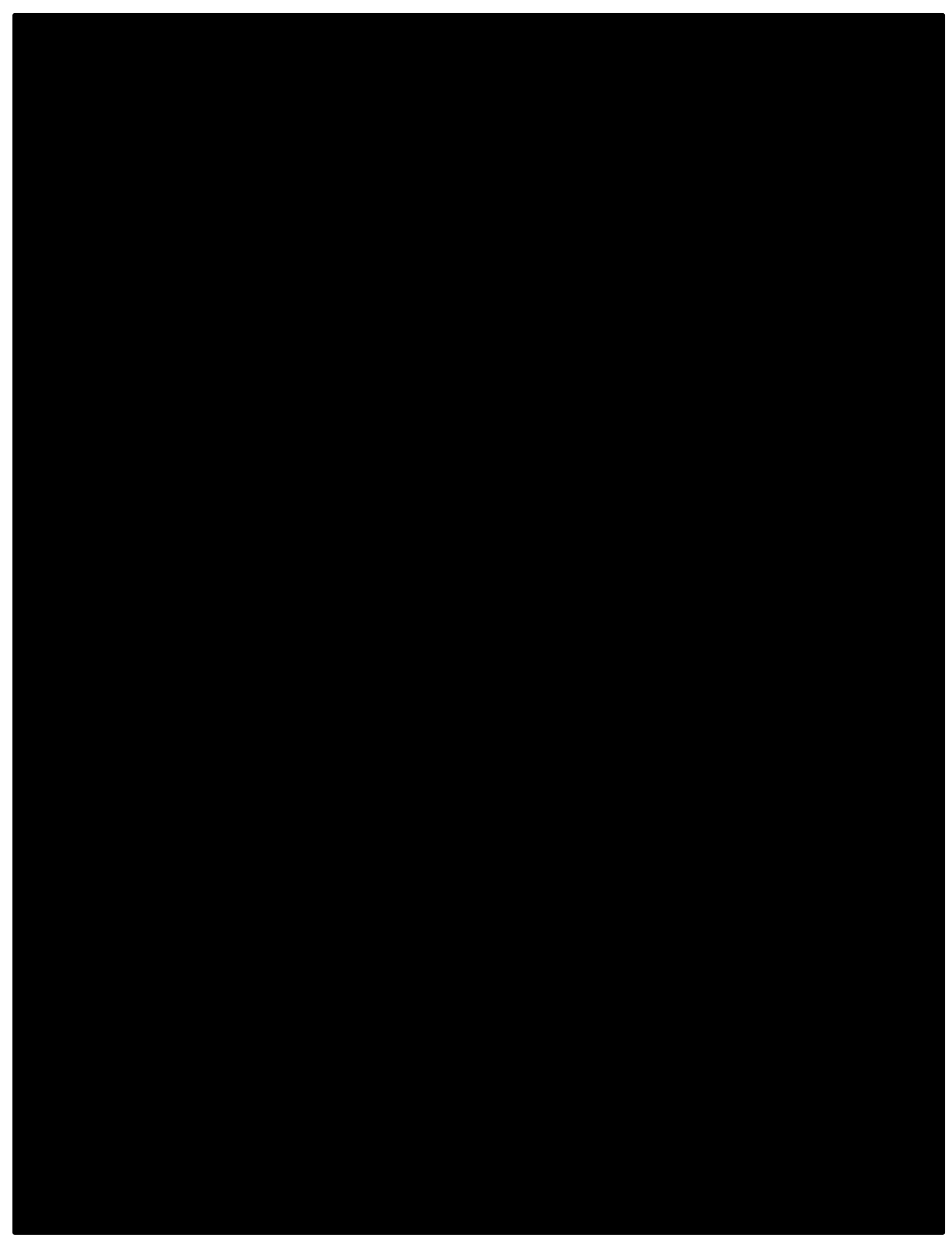

Figure A1 - Corrected pDNR-Dual hCLU plasmid

Clusterin cloned between sites Sall and Xbal. Overall plasmid size is $625 \mathrm{~kb}$ (including linkers on primers added when undergoing PCR amplification). 


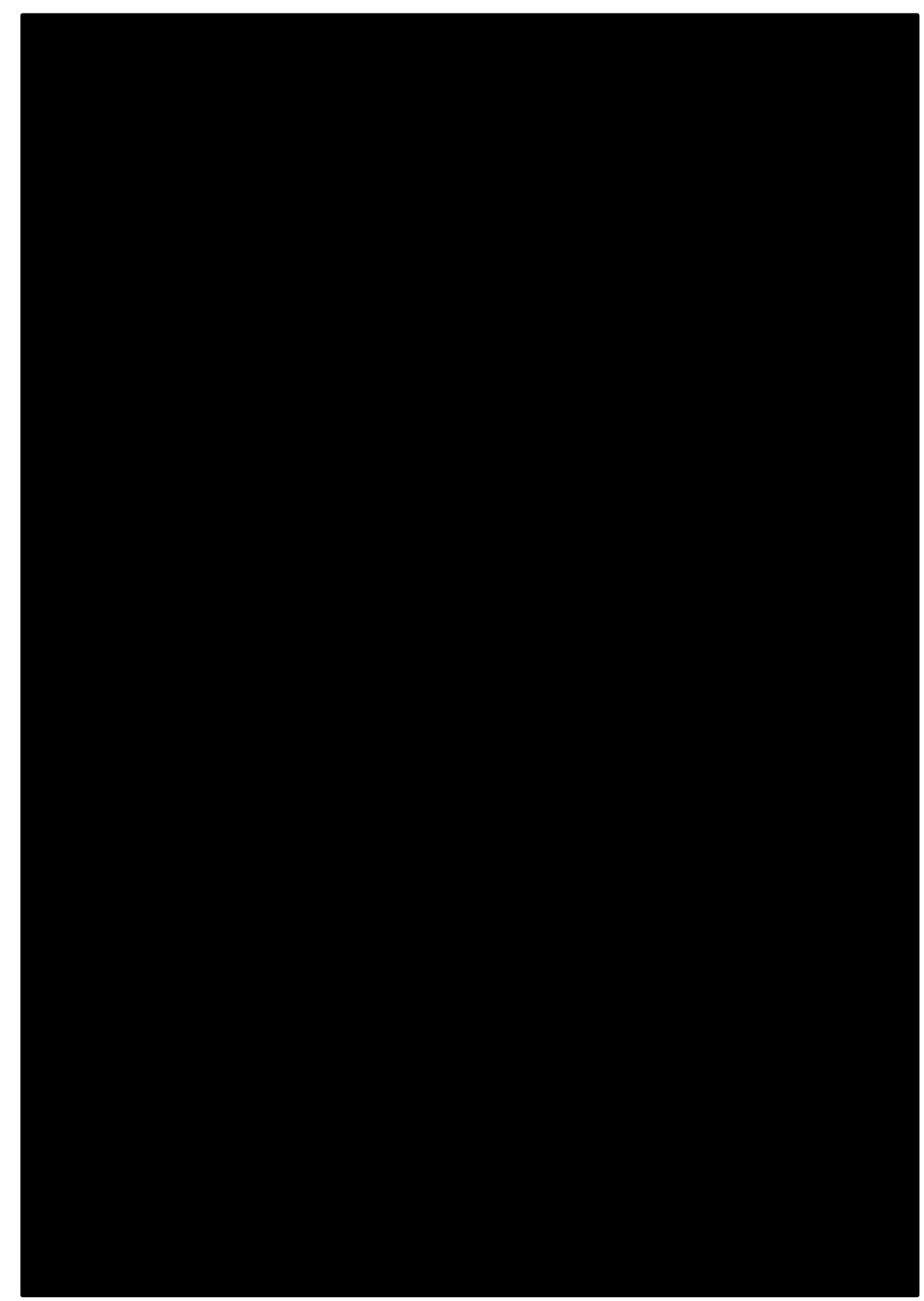

Figure A2 - pUAST-attB plasmid

Unique sites in the polylinker are EcoRI, BgllI, Notl, Xhol, Kpnl and Xbal (Figure S1). attB is the sequence for homologous recombination with attP, which is inserted into the genome of the strain that is used for microinjection. 


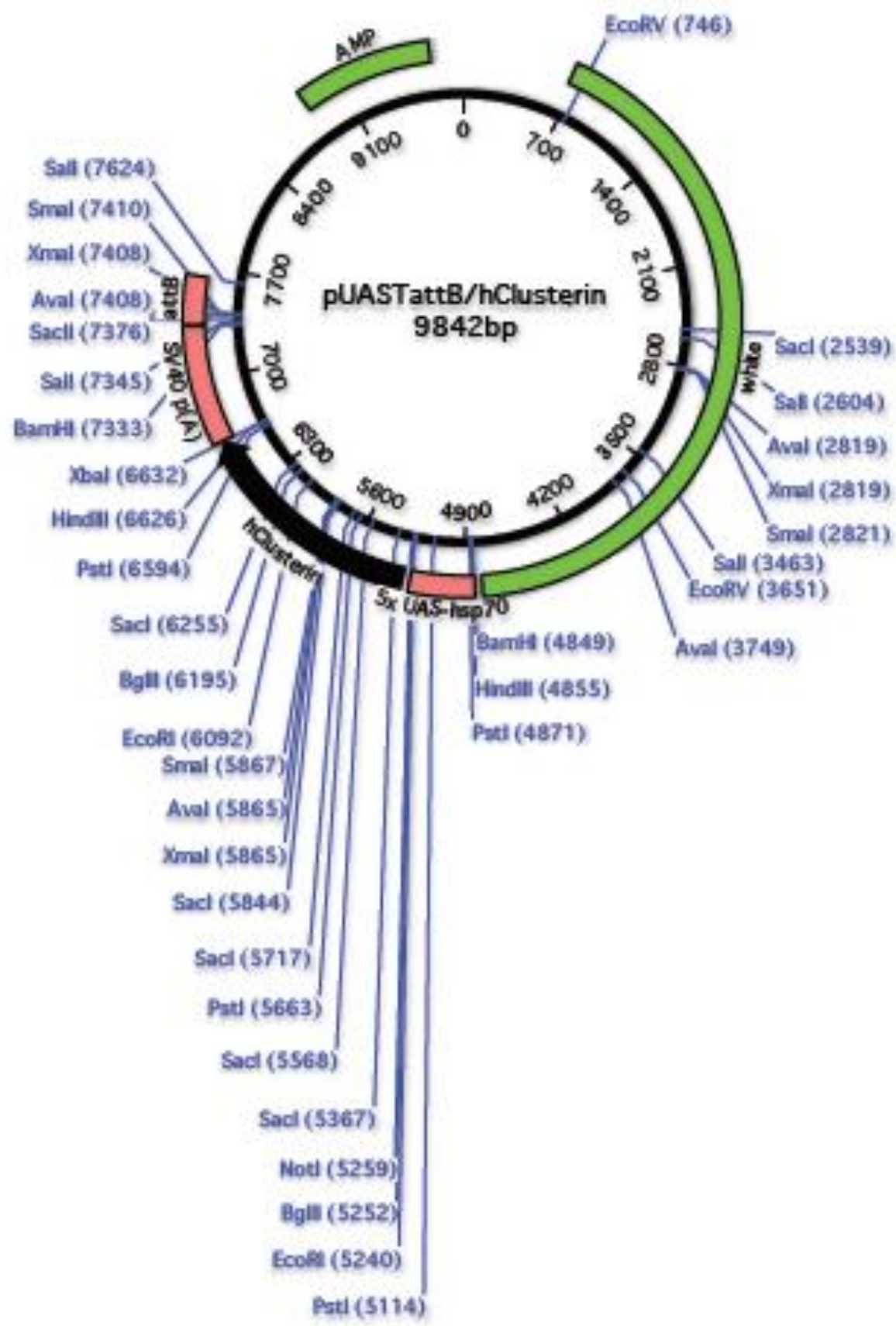

Figure A3 - pUAST-attb hCLU plasmid

Clusterin is cloned between sites UAS and attb-SV40. This construct was generated for microinjection into drosophila eggs to create flies that over-express the clusterin gene in the brain as well as throughout the whole CNS of the fly. 


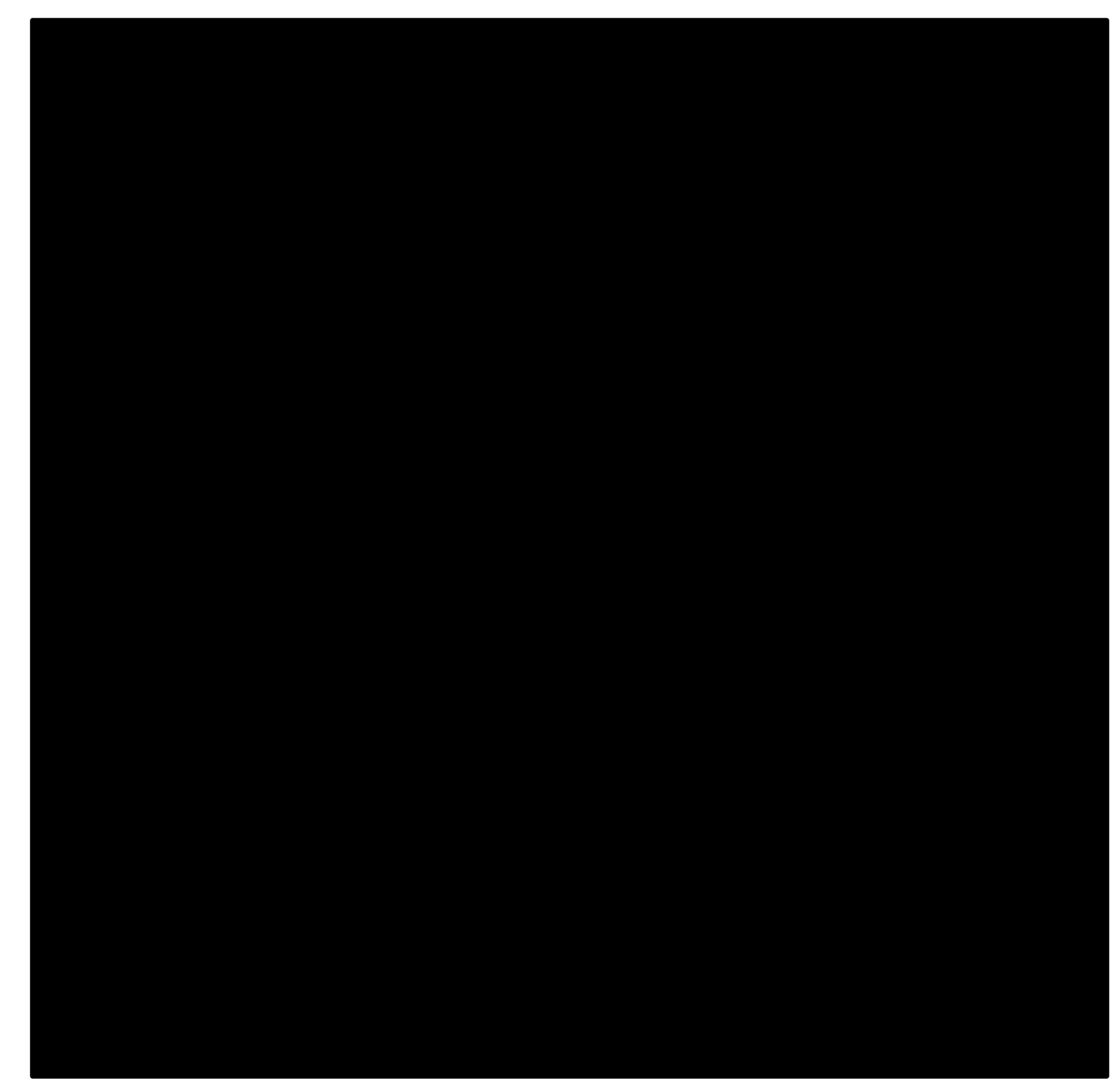

Figure A4 - pcDNA3.1(+) plasmid

Plasmid used as a vector for inserting CLU DNA from pUAST-attB (Figure A3) to generate into pcDNA3.1(+)/CLU. 

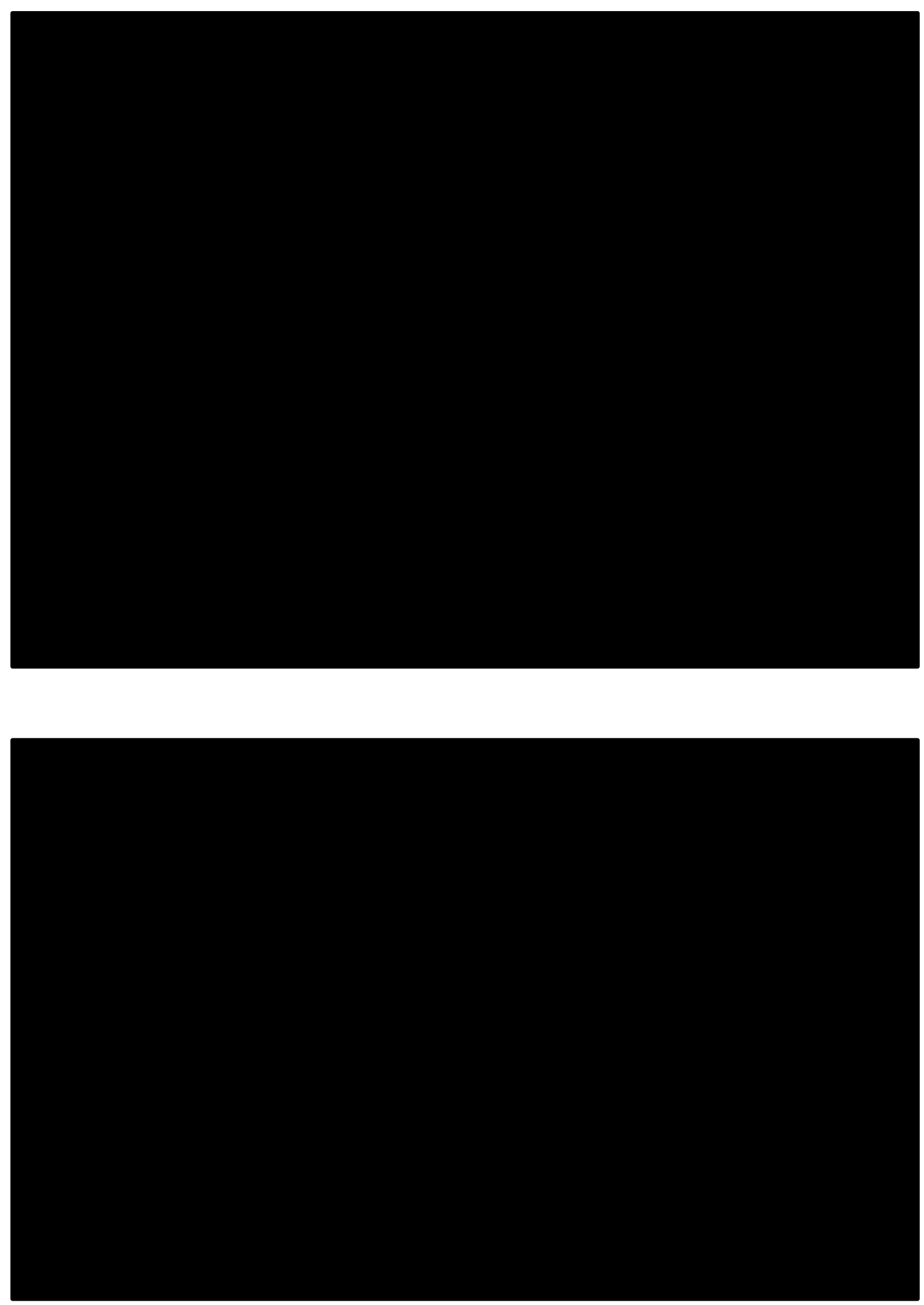

Figure A5 - RG203629 GFP plasmid

Plasmid used to cleave GFP sequence out of to insert into pcDNA3.1(+)/CLU vector. 